\title{
Catalytic Asymmetric Diamination of Conjugated Dienes and Triene
}

\author{
Haifeng Du, Weicheng Yuan, Baoguo Zhao, and Yian Shi*
}

Department of Chemistry

Colorado State University

Fort Collins, CO 80523

Supporting Information 
Representative catalytic asymmetric diamination procedure (Table 1, entry 1). An NMR tube charged with $\mathrm{Pd}_{2}(\mathrm{dba})_{3}(0.0183 \mathrm{~g}, 0.020 \mathrm{mmol})$ and $\mathbf{L 7}(0.040 \mathrm{~g}, 0.088 \mathrm{~mol})$ was evacuated and then filled with argon three times. Benzene- $d_{6}(0.20 \mathrm{~mL}$, distilled from sodium $)$ was added, and the resulting mixture was then immersed into an oil bath $\left(65^{\circ} \mathrm{C}\right)$. The NMR tube was shaken occasionally until solid was dissolved (ca. $10 \mathrm{~min})$. After trans-1,3-pentadiene (0.0272 g, $0.40 \mathrm{mmol})$ and di-t-butyldiaziridinone $(0.085 \mathrm{~g}, 0.50 \mathrm{mmol})$ were added sequentially, the resulting mixture was immersed into an oil bath $\left(65^{\circ} \mathrm{C}\right)$ until completion or no further reaction detected $(1.5 \mathrm{~h})$, and purified by flash chromatography (silica gel, hexane:ethyl actate $=5: 1)$ to give compound containing with small amount of dba which was further purified by flash chromatography (silica gel, toluene then hexane:ethyl actate $=5: 1$ ) to give the diamination product as a colorless oil ( $0.087 \mathrm{~g}, 91 \%$ yield, $91 \% \mathrm{ee})$.

(note: the purities of olefin substrates used in this process are very important for good conversions. They are freshly prepared and purified.)

Preparation of compound 7 (Scheme 3). To a $10 \mathrm{~mL}$ vial, was added compound 6 (0.785 g, $2.5 \mathrm{mmol})$, followed by the addition of $\mathrm{CF}_{3} \mathrm{CO}_{2} \mathrm{H}(5.0 \mathrm{~mL})$. Upon stirring at $80{ }^{\circ} \mathrm{C}$ for $1.0 \mathrm{~h}$, the reaction mixture was concentrated and purified by flash chromatography (silica gel, ethyl acetate/methanol 15/1) to give compound 7 as a white solid(0.465 g, 92\% yield). Compound 7 $(0.450 \mathrm{~g})$ was recrystallized in hexane/acetone to give a white solid $(0.272 \mathrm{~g}, 60 \%$ yield, $>98 \%$ ee).

Preparation of compound 8 (Scheme 3). A solution of compound 7 (0.0808 g, $0.40 \mathrm{mmol})$ in conc. $\mathrm{HCl}(10 \mathrm{~mL})$ was stirred at reflux for $24 \mathrm{~h}$, concentrated under reduced pressure, diluted with water $(10 \mathrm{~mL})$, and washed with $\mathrm{CH}_{2} \mathrm{Cl}_{2}(3 \times 10 \mathrm{~mL})$. The aqueous solution was adjusted to basic $(\mathrm{pH}>12)$ with $\mathrm{NaOH}(1 \mathrm{~N})$ and extracted with $\mathrm{CH}_{2} \mathrm{Cl}_{2}(3 \times 10 \mathrm{~mL})$. The organic layers were dried $\left(\mathrm{Na}_{2} \mathrm{SO}_{4}\right)$, filtered, and concentrated to give diamine $\mathbf{8}$ as dark yellow oil $(0.0633 \mathrm{~g}, 90 \%$ yield, $>98 \%$ ee).

Preparation of compound 9 (Scheme 3). Compound 9 was prepared according to the literature procedure with slight modification (Oshitari, T.; Akagi, R.; Mandai, T. Synthesis, 2004, 1325). Compound $6(0.1884 \mathrm{~g}, 0.60 \mathrm{mmol})$ in 1,4-dioxane $(14.0 \mathrm{~mL}) / \mathrm{H}_{2} \mathrm{O}(4.5 \mathrm{~mL})$ was treated with 
$\mathrm{Me}_{3} \mathrm{NO} \cdot 2 \mathrm{H}_{2} \mathrm{O}(0.1332 \mathrm{~g}, 1.20 \mathrm{mmol})$ and aq. $\mathrm{OsO}_{4}(0.0089 \mathrm{~g}, 0.035 \mathrm{mmol}, 0.22 \mathrm{~mL} 4 \% \mathrm{w} / \mathrm{w})$ at $\mathrm{rt}$ for $14 \mathrm{~h}$. Upon addition of $\mathrm{NaIO}_{4}(0.4494 \mathrm{~g}, 2.10 \mathrm{mmol})$, the reaction mixture was stirred at $\mathrm{rt}$ for $2 \mathrm{~h}$, quenched with sat. aq. $\mathrm{Na}_{2} \mathrm{~S}_{2} \mathrm{O}_{3}$, and extracted with ethyl acetate. The organic layers were dried $\left(\mathrm{MgSO}_{4}\right)$ and concentrated. The residue was dissolved in $t-\mathrm{BuOH}(14.0 \mathrm{~mL}) / \mathrm{H}_{2} \mathrm{O}$ $(4.5 \mathrm{~mL})$, and treated with $\mathrm{NaClO}_{2}(0.0814 \mathrm{~g}, 0.90 \mathrm{mmol})$ in the presence of $\mathrm{NaH}_{2} \mathrm{PO}_{4}(0.144 \mathrm{~g}$, $1.2 \mathrm{mmol})$ and 2-methyl-2-butene $(0.42 \mathrm{~g}, 6.0 \mathrm{mmol})$ at $\mathrm{rt}$ for $20 \mathrm{~h}$. The reaction mixture was diluted with toluene and extracted with aq $\mathrm{Na}_{2} \mathrm{CO}_{3}(10 \%)$. The aqueous layer was acidified with conc. $\mathrm{HCl}$ and extracted with ethyl acetate. The organic layers were washed with brine, dried $\left(\mathrm{MgSO}_{4}\right)$, and concentrated to afford compound 9 as a white solid $(0.146 \mathrm{~g}, 73 \%$ yield for 3 steps, $92 \%$ ee).

Preparation of compound 10 (Scheme 3). To a $3.0 \mathrm{~mL}$ vial was added compound 9 (0.166 g, $0.50 \mathrm{mmol})$, followed by the addition of $\mathrm{CF}_{3} \mathrm{CO}_{2} \mathrm{H}(1.0 \mathrm{~mL})$. Upon stirring at $80{ }^{\circ} \mathrm{C}$ for $1.0 \mathrm{~h}$, the reaction mixture was concentrated, and the residue was treated with $\mathrm{Et}_{2} \mathrm{O}$ and filtered to give compound 10 (0.1044 g, 95\% yield, 92\% ee). Compound 10 (0.060 g) was recrystallized in ${ }^{i} \mathrm{PrOH}$ to give a white solid $(0.040 \mathrm{~g}, 67 \%$ yield, $>99 \%$ ee).

Preparation of compound 11 (Scheme 3). Compound 11 was prepared according to the literature procedure (Dunn, P.J; Häner, R.; Rapoport, H. J. Org. Chem. 1990, 55, 5017). To a 3.0 $\mathrm{mL}$ vial was added compound $10(0.033 \mathrm{~g}, 0.15 \mathrm{mmol},>99 \%$ ee $)$, followed by $2 \mathrm{~N} \mathrm{HCl}(0.5 \mathrm{~mL})$. Upon stirring at $100{ }^{\circ} \mathrm{C}$ for $2.5 \mathrm{~h}$, the reaction mixture was concentrated and dried under vacuum to give compound $\mathbf{1 1}$ as white solid $(0.0396 \mathrm{~g},>99 \%$ yield $)$.

Preparation of compound 12 (Scheme 3). To a $3.0 \mathrm{~mL}$ vial was added compound 9 (0.0332 g, $0.10 \mathrm{mmol})$, followed by $\mathrm{CF}_{3} \mathrm{CO}_{2} \mathrm{H}(0.2 \mathrm{~mL})$. Upon stirring at $\mathrm{rt}$ for $1.0 \mathrm{~h}$, the reaction mixture was concentrated at $\mathrm{rt}$ to give compound $12(0.0227 \mathrm{~g},>99 \%$ yield, $92 \%$ ee $)$. 


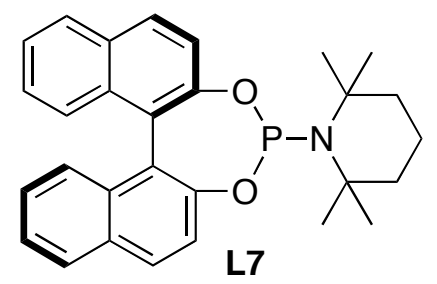

White solid; $\operatorname{mp} 210-21{ }^{\circ} \mathrm{C} ;[\alpha]^{20}{ }_{\mathrm{D}}=-352.9\left(c 0.785, \mathrm{CHCl}_{3}\right)$; IR (film) 1618, 1592, 1507, $1463 \mathrm{~cm}^{-1}$; ${ }^{1} \mathrm{H}$ NMR $\left(300 \mathrm{MHz}\right.$, benezene- $\left.d_{6}\right) \delta 7.64(\mathrm{dd}, J=10.5,9.0 \mathrm{~Hz}, 4 \mathrm{H}), 7.50-7.40(\mathrm{~m}$, 4H), 7.20-7.08 (m, 2H), 6.90 (dd, $J=15.6,7.5 \mathrm{~Hz}, 2 \mathrm{H}), 1.56(\mathrm{~s}, 6 \mathrm{H}), 1.50-1.20(\mathrm{~m}, 12 \mathrm{H}) ;{ }^{31} \mathrm{P}$ NMR $\left(121 \mathrm{MHz}\right.$, benezene- $\left.d_{6}\right) \delta 164.1$; Anal. Calcd for $\mathrm{C}_{29} \mathrm{H}_{30} \mathrm{NO}_{2} \mathrm{P}: \mathrm{C}, 76.46 ; \mathrm{H}, 6.64 ; \mathrm{N}$, 3.07. Found: C, 76.60; H, 6.80; N, 2.95 .

\section{Table 1, Entry 1}

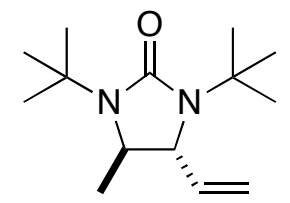

Colorless oil; $[\alpha]^{20}{ }_{\mathrm{D}}=+31.5\left(c 0.835, \mathrm{CHCl}_{3}\right)(91 \%$ ee $)$; IR (film) $1689 \mathrm{~cm}^{-1} ;{ }^{1} \mathrm{H}$ NMR $(300$ $\left.\mathrm{MHz}, \mathrm{CDCl}_{3}\right) \delta 5.92(\mathrm{ddd}, J=17.1,10.2,8.4 \mathrm{~Hz}, 1 \mathrm{H}), 5.19$ (d, $\left.J=17.1 \mathrm{~Hz}, 1 \mathrm{H}\right), 5.09$ (d, $J=$ $10.2 \mathrm{~Hz}, 1 \mathrm{H}), 3.51$ (d, $J=8.4 \mathrm{~Hz}, 1 \mathrm{H}), 3.27$ (q, $J=6.0 \mathrm{~Hz}, 1 \mathrm{H}), 1.36$ (s, 9H), 1.35 (s, 9H), 1.21 $(\mathrm{d}, J=6.0 \mathrm{~Hz}, 3 \mathrm{H}) ;{ }^{13} \mathrm{C} \mathrm{NMR}\left(75 \mathrm{MHz}, \mathrm{CDCl}_{3}\right) \delta 157.9,140.0,115.6,63.6,55.4,53.1,52.5$, 29.1, 28.9, 21.2.

Du, H.; Zhao, B.; Shi, Y. J. Am. Chem. Soc., 2007, 129, 762.

Table 1, Entry 2

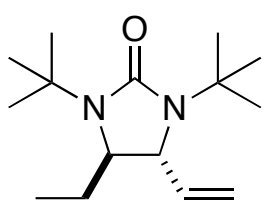


Colorless oil; $[\alpha]^{20}{ }_{\mathrm{D}}=+8.1\left(\mathrm{c} 1.13, \mathrm{CHCl}_{3}\right)\left(92 \%\right.$ ee); IR (film) $1689 \mathrm{~cm}^{-1} ;{ }^{1} \mathrm{H} \mathrm{NMR}(300 \mathrm{MHz}$, $\left.\mathrm{CDCl}_{3}\right) \delta 5.92(\mathrm{ddd}, J=17.1,10.2,8.1 \mathrm{~Hz}, 1 \mathrm{H}), 5.18(\mathrm{~d}, J=17.1 \mathrm{~Hz}, 1 \mathrm{H}), 5.08(\mathrm{~d}, J=10.2 \mathrm{~Hz}$, $1 \mathrm{H}), 3.67$ (d, $J=8.1 \mathrm{~Hz}, 1 \mathrm{H}), 3.05$ (dd, $J=6.0,5.4 \mathrm{~Hz}, 1 \mathrm{H}), 1.64-1.50(\mathrm{~m}, 2 \mathrm{H}), 1.36(\mathrm{~s}, 9 \mathrm{H})$, $1.35(\mathrm{~s}, 9 \mathrm{H}), 0.91(\mathrm{t}, J=7.5 \mathrm{~Hz}, 3 \mathrm{H}) ;{ }^{13} \mathrm{C} \mathrm{NMR}\left(75 \mathrm{MHz}, \mathrm{CDCl}_{3}\right) \delta 158.3,140.8,115.1,60.9$, 60.2, 53.1, 52.6, 29.1, 29.0, 27.2, 9.1.

Du, H.; Zhao, B.; Shi, Y. J. Am. Chem. Soc., 2007, 129, 762.

Table 1, Entry 3

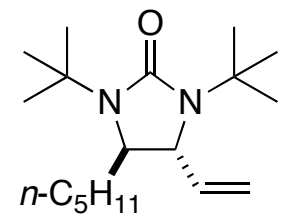

Colorless oil; $[\alpha]^{20}{ }_{\mathrm{D}}=+6.4\left(c 0.94, \mathrm{CHCl}_{3}\right)\left(92 \%\right.$ ee); IR (film) $1690 \mathrm{~cm}^{-1} ;{ }^{1} \mathrm{H} \mathrm{NMR}(300 \mathrm{MHz}$, $\left.\mathrm{CDCl}_{3}\right) \delta 5.91(\mathrm{ddd}, J=17.1,10.2,8.4 \mathrm{~Hz}, 1 \mathrm{H}), 5.18(\mathrm{~d}, J=17.1 \mathrm{~Hz}, 1 \mathrm{H}), 5.08(\mathrm{~d}, J=10.2 \mathrm{~Hz}$, $1 \mathrm{H}), 3.66(\mathrm{~d}, J=8.4 \mathrm{~Hz}, 1 \mathrm{H}), 3.10(\mathrm{dd}, J=8.4,3.0 \mathrm{~Hz}, 1 \mathrm{H}), 1.60-1.44(\mathrm{~m}, 2 \mathrm{H}), 1.36(\mathrm{~s}, 9 \mathrm{H})$, $1.34(\mathrm{~s}, 9 \mathrm{H}), 1.42-1.18(\mathrm{~m}, 6 \mathrm{H}), 0.90(\mathrm{t}, J=6.9 \mathrm{~Hz}, 3 \mathrm{H}) ;{ }^{13} \mathrm{C} \mathrm{NMR}\left(75 \mathrm{MHz}, \mathrm{CDCl}_{3}\right) \delta 158.3$, $140.7,115.1,60.6,59.7,53.1,52.6,34.3,32.0,29.1,29.0,24.6,22.8,14.1$.

Du, H.; Zhao, B.; Shi, Y. J. Am. Chem. Soc., 2007, 129, 762.

Table 1, Entry 4

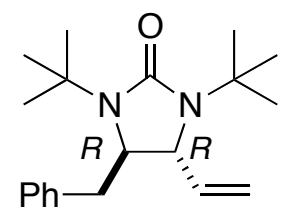

Colorless oil; $[\alpha]_{\mathrm{D}}^{20}=-21.5\left(c 0.71, \mathrm{CHCl}_{3}\right)\left(92 \%\right.$ ee); IR (film) $1689 \mathrm{~cm}^{-1} ;{ }^{1} \mathrm{H}$ NMR $(300$ $\left.\mathrm{MHz}, \mathrm{CDCl}_{3}\right) \delta$ 7.40-7.30 (m, 2H), 7.24-7.20 (m, 1H), 7.20-7.12 (m, 2H), 5.78 (ddd, J = 17.1, $10.2,8.1 \mathrm{~Hz}, 1 \mathrm{H}), 4.95(\mathrm{~d}, J=10.2 \mathrm{~Hz}, 1 \mathrm{H}), 4.85(\mathrm{~d}, J=17.1 \mathrm{~Hz}, 1 \mathrm{H}), 3.64(\mathrm{~d}, J=8.1 \mathrm{~Hz}, 1 \mathrm{H})$, $3.41(\mathrm{dd}, J=9.9,3.3 \mathrm{~Hz}, 1 \mathrm{H}), 3.01(\mathrm{dd}, J=13.5,3.3 \mathrm{~Hz}, 1 \mathrm{H}), 2.67(\mathrm{dd}, J=13.5,9.9 \mathrm{~Hz}, 1 \mathrm{H})$, $1.46(\mathrm{~s}, 9 \mathrm{H}), 1.30(\mathrm{~s}, 9 \mathrm{H}) ;{ }^{13} \mathrm{C} \mathrm{NMR}\left(75 \mathrm{MHz}, \mathrm{CDCl}_{3}\right) \delta 158.2,139.9,137.6,129.2,128.8$, 
126.8, 115.5, 60.9, 58.9, 53.0, 52.9, 40.1, 29.2, 28.9; HRMS Calcd for $\mathrm{C}_{20} \mathrm{H}_{31} \mathrm{~N}_{2} \mathrm{O}(\mathrm{M}+1)$ : 315.2431. Found: 315.2423 .

\section{Table 1, Entry 5}

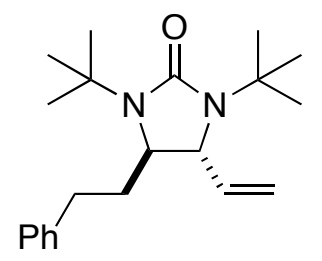

Colorless oil; $[\alpha]^{20}{ }_{\mathrm{D}}=+10.4\left(c 0.615, \mathrm{CHCl}_{3}\right)(92 \%$ ee $)$; IR (film) $1687 \mathrm{~cm}^{-1} ;{ }^{1} \mathrm{H}$ NMR $(300$ $\left.\mathrm{MHz}, \mathrm{CDCl}_{3}\right) \delta$ 7.34-7.26 (m, 2H), 7.24-7.14 (m, 3H), 5.91 (ddd, $J=17.4,10.5,8.4 \mathrm{~Hz}, 1 \mathrm{H}$ ), $5.19(\mathrm{~d}, J=17.4 \mathrm{~Hz}, 1 \mathrm{H}), 5.11(\mathrm{~d}, J=10.5 \mathrm{~Hz}, 1 \mathrm{H}), 3.73(\mathrm{~d}, J=8.4 \mathrm{~Hz}, 1 \mathrm{H}), 3.17$ (dd, $J=8.4$, $2.4 \mathrm{~Hz}, 1 \mathrm{H}), 2.80-2.68(\mathrm{~m}, 1 \mathrm{H}), 2.66-2.54(\mathrm{~m}, 1 \mathrm{H}), 2.00-1.78(\mathrm{~m}, 2 \mathrm{H}), 1.35(\mathrm{~s}, 9 \mathrm{H}), 1.33(\mathrm{~s}$, $9 \mathrm{H}) ;{ }^{13} \mathrm{C}$ NMR $\left(75 \mathrm{MHz}, \mathrm{CDCl}_{3}\right) \delta 158.3,141.4,140.2,128.7,128.5,126.2,115.5,60.6,59.0$, 53.1, 52.7, 35.9, 31.3, 29.1, 28.9; HRMS Calcd for $\mathrm{C}_{21} \mathrm{H}_{33} \mathrm{~N}_{2} \mathrm{O}(\mathrm{M}+1)$ : 329.2587. Found: 329.2589 .

\section{Table 1, Entry 6}

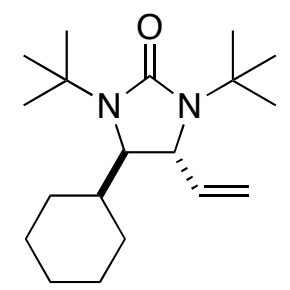

Colorless oil; $[\alpha]^{20}{ }_{\mathrm{D}}=+20.9\left(c 0.56, \mathrm{CHCl}_{3}\right)(95 \%$ ee $) ; \operatorname{IR}($ film $) 1687 \mathrm{~cm}^{-1} ;{ }^{1} \mathrm{H}$ NMR (300 $\left.\mathrm{MHz}, \mathrm{CDCl}_{3}\right) \delta 5.88(\mathrm{ddd}, J=17.1,10.2,8.4 \mathrm{~Hz}, 1 \mathrm{H}), 5.14(\mathrm{~d}, J=17.1 \mathrm{~Hz}, 1 \mathrm{H}), 5.05(\mathrm{~d}, J=$ $10.2 \mathrm{~Hz}, 1 \mathrm{H}), 3.79$ (d, $J=8.4 \mathrm{~Hz}, 1 \mathrm{H}), 3.00(\mathrm{~d}, J=2.7 \mathrm{~Hz}, 1 \mathrm{H}), 1.88-1.68(\mathrm{~m}, 4 \mathrm{H}), 1.62-1.48$ (m, 2H), 1.37 (s, 9H), 1.34 (s, 9H), 1.32-0.88 (m, 5H); $\left.{ }^{13} \mathrm{C} \mathrm{NMR} \mathrm{(75} \mathrm{MHz,} \mathrm{CDCl}_{3}\right) \delta$ 158.7, 141.4, 114.7, 64.1, 57.0, 53.2, 52.8, 43.5, 29.1, 28.9, 26.8, 26.7, 26.6, 26.2; Anal. Calcd for $\mathrm{C}_{19} \mathrm{H}_{34} \mathrm{~N}_{2} \mathrm{O}:$ C, 74.46; H, 11.18; N, 9.14. Found: C, 74.50; H, 11.32; N, 9.19. 
Table 1, Entry 7

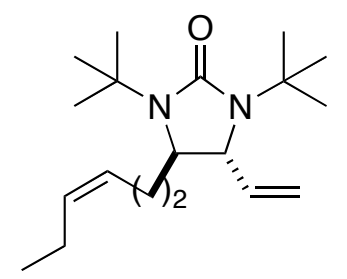

Colorless oil; $[\alpha]^{20}{ }_{\mathrm{D}}=+6.1\left(c 0.76, \mathrm{CHCl}_{3}\right)(93 \%$ ee $)$; IR (film) $1690 \mathrm{~cm}^{-1} ;{ }^{1} \mathrm{H} \mathrm{NMR}(300 \mathrm{MHz}$, $\left.\mathrm{CDCl}_{3}\right) \delta 5.91(\mathrm{ddd}, J=17.1,10.2,8.1 \mathrm{~Hz}, 1 \mathrm{H}), 5.50-5.26(\mathrm{~m}, 2 \mathrm{H}), 5.19(\mathrm{~d}, J=17.1 \mathrm{~Hz}, 1 \mathrm{H})$, $5.19(\mathrm{~d}, J=10.2 \mathrm{~Hz}, 1 \mathrm{H}), 3.70(\mathrm{~d}, J=8.1 \mathrm{~Hz}, 1 \mathrm{H}), 3.13(\mathrm{dd}, J=8.7,3.0 \mathrm{~Hz}, 1 \mathrm{H}), 2.20-1.96$ (m, 4H), 1.72-1.50 (m, 2H), $1.36(\mathrm{~s}, 9 \mathrm{H}), 1.35(\mathrm{~s}, 9 \mathrm{H}), 0.96(\mathrm{t}, J=7.5 \mathrm{~Hz}, 3 \mathrm{H}) ;{ }^{13} \mathrm{C} \mathrm{NMR}(75 \mathrm{MHz}$, $\left.\mathrm{CDCl}_{3}\right) \delta 158.3,140.4,132.8,127.8,115.4,60.6,59.2,53.1,52.7,34.0,29.2,29.0,22.6,20.7$, 14.5; HRMS Calcd for $\mathrm{C}_{19} \mathrm{H}_{35} \mathrm{~N}_{2} \mathrm{O}(\mathrm{M}+1)$ : 307.2744. Found: 307.2743.

\section{Table 1, Entry 8}

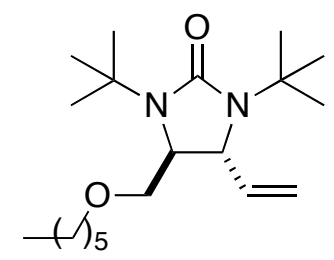

Colorless oil; $[\alpha]_{\mathrm{D}}^{20}=-4.5\left(c 1.08, \mathrm{CHCl}_{3}\right)\left(92 \%\right.$ ee); IR (film) $1693 \mathrm{~cm}^{-1} ;{ }^{1} \mathrm{H}$ NMR (300 MHz, $\left.\mathrm{CDCl}_{3}\right) \delta 5.91(\mathrm{ddd}, J=17.1,10.2,7.5 \mathrm{~Hz}, 1 \mathrm{H}), 5.23(\mathrm{~d}, J=17.1 \mathrm{~Hz}, 1 \mathrm{H}), 5.11(\mathrm{~d}, J=10.2 \mathrm{~Hz}$, $1 \mathrm{H}), 3.96(\mathrm{~d}, J=7.5 \mathrm{~Hz}, 1 \mathrm{H}), 3.54-3.22(\mathrm{~m}, 5 \mathrm{H}), 1.60-1.50(\mathrm{~m}, 2 \mathrm{H}), 1.42-1.24(\mathrm{~m}, 6 \mathrm{H}), 1.34(\mathrm{~s}$, $18 \mathrm{H}), 0.88(\mathrm{t}, J=6.3 \mathrm{~Hz}, 3 \mathrm{H}) ;{ }^{13} \mathrm{C} \mathrm{NMR}\left(75 \mathrm{MHz}, \mathrm{CDCl}_{3}\right) \delta 158.6,139.9,115.8,71.6,71.1$, 58.8, 58.5, 53.1, 52.7, 31.8, 29.7, 29.1, 28.9, 26.0, 22.8, 14.2.

Du, H.; Zhao, B.; Shi, Y. J. Am. Chem. Soc., 2007, 129, 762. 


\section{Table 1, Entry 9}

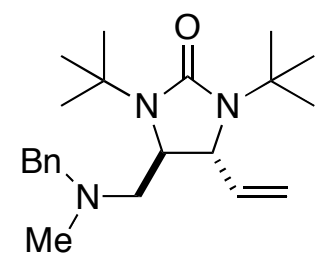

Colorless oil; $[\alpha]_{\mathrm{D}}^{20}=-62.4\left(c 0.68, \mathrm{CHCl}_{3}\right)\left(92 \%\right.$ ee); IR (film) $1688 \mathrm{~cm}^{-1} ;{ }^{1} \mathrm{H}$ NMR $(300$ $\left.\mathrm{MHz}, \mathrm{CDCl}_{3}\right) \delta$ 7.40-7.24 (m, 5H), $5.90(\mathrm{ddd}, J=17.1,10.2,7.8 \mathrm{~Hz}, 1 \mathrm{H}), 5.24(\mathrm{~d}, J=17.1 \mathrm{~Hz}$, $1 \mathrm{H}), 5.10$ (d, $J=10.2 \mathrm{~Hz}, 1 \mathrm{H}), 3.99$ (d, $J=7.8 \mathrm{~Hz}, 1 \mathrm{H}), 3.67$ (d, $J=12.9 \mathrm{~Hz}, 1 \mathrm{H}), 3.28(\mathrm{~d}, J=$ $12.9 \mathrm{~Hz}, 1 \mathrm{H}), 3.22(\mathrm{dd}, J=10.8,3.0 \mathrm{~Hz}, 1 \mathrm{H}), 2.48(\mathrm{dd}, J=12.6,10.8 \mathrm{~Hz}, 1 \mathrm{H}), 2.28$ (s, 3H), $2.20(\mathrm{dd}, J=12.6,3.0 \mathrm{~Hz}, 1 \mathrm{H}), 1.33(\mathrm{~s}, 9 \mathrm{H}), 1.19(\mathrm{~s}, 9 \mathrm{H}) ;{ }^{13} \mathrm{C} \mathrm{NMR}\left(75 \mathrm{MHz}, \mathrm{CDCl}_{3}\right) \delta 158.5$, 140.6, 139.1, 129.3, 128.5, 127.4, 115.4, 63.4, 58.8, 58.7, 57.9, 52.9, 52.6, 43.6, 29.2, 28.7; Anal. Calcd for $\mathrm{C}_{22} \mathrm{H}_{35} \mathrm{~N}_{3} \mathrm{O}$ : C, 73.91; H, 9.87; N, 11.75. Found: C, 73.91; H, 9.85; N, 11.59.

\section{Table 1, Entry 10}

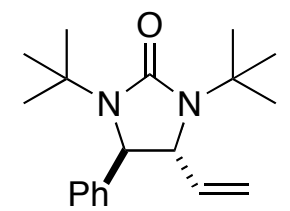

Colorless oil; $[\alpha]_{\mathrm{D}}^{20}=+30.2\left(c 0.60, \mathrm{CHCl}_{3}\right)\left(93 \%\right.$ ee); IR (film) $1689 \mathrm{~cm}^{-1} ;{ }^{1} \mathrm{H}$ NMR $(300$ $\left.\mathrm{MHz} \mathrm{CDCl}_{3}\right) \delta$ 7.40-7.24 (m, 5H), $6.03(\mathrm{ddd}, J=17.7,10.2,8.1 \mathrm{~Hz}, 1 \mathrm{H}), 5.19$ (d, J=17.7 Hz, $1 \mathrm{H}), 5.15(\mathrm{~d}, J=10.2 \mathrm{~Hz}, 1 \mathrm{H}), 4.16(\mathrm{~s}, 1 \mathrm{H}), 3.66(\mathrm{~d}, J=8.1 \mathrm{~Hz}, 1 \mathrm{H}), 1.34$ (s, 9H), 1.28 (s, 9H); ${ }^{13} \mathrm{C}$ NMR $\left(75 \mathrm{MHz}, \mathrm{CDCl}_{3}\right) \delta 159.1,144.0,140.9,128.9,127.9,125.9,115.8,64.9,63.3,53.7$, $53.4,29.0,28.8$.

Du, H.; Zhao, B.; Shi, Y. J. Am. Chem. Soc., 2007, 129, 762. 


\section{Table 1, Entry 11}

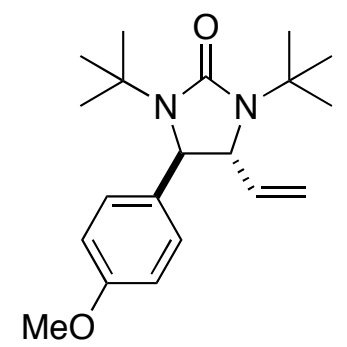

Colorless oil; $[\alpha]_{\mathrm{D}}^{20}=+54.0\left(c 0.57, \mathrm{CHCl}_{3}\right)\left(92 \%\right.$ ee); IR (film) $1687 \mathrm{~cm}^{-1} ;{ }^{1} \mathrm{H}$ NMR $(300$ $\left.\mathrm{MHz}, \mathrm{CDCl}_{3}\right) \delta$ 7.24-7.18 (m, 2H), 6.92-6.84 (m, 2H), $6.02(\mathrm{ddd}, J=17.1,10.2,8.4 \mathrm{~Hz}, 1 \mathrm{H})$, $5.19(\mathrm{~d}, J=17.1 \mathrm{~Hz}, 1 \mathrm{H}), 5.15(\mathrm{~d}, J=10.2 \mathrm{~Hz}, 1 \mathrm{H}), 4.11(\mathrm{~s}, 1 \mathrm{H}), 3.81(\mathrm{~s}, 3 \mathrm{H}), 3.63(\mathrm{~d}, J=8.4$ $\mathrm{Hz}, 1 \mathrm{H}), 1.34$ (s, 9H), 1.27 (s, 9H); ${ }^{13} \mathrm{C} \mathrm{NMR}\left(75 \mathrm{MHz}, \mathrm{CDCl}_{3}\right) \delta$ 159.2, 159.0, 140.8, 136.1, 127.0, 115.7, 114.1, 65.1, 62.7, 55.3, 53.5, 53.3, 28.9, 28.8.

Du, H.; Zhao, B.; Shi, Y. J. Am. Chem. Soc., 2007, 129, 762.

\section{Table 1, Entry 12}

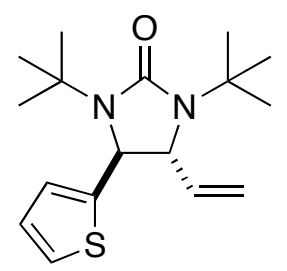

Colorless oil; $[\alpha]^{20}{ }_{\mathrm{D}}=+67.7\left(c 0.22, \mathrm{CHCl}_{3}\right)\left(93 \%\right.$ ee); IR (film) $1692 \mathrm{~cm}^{-1} ;{ }^{1} \mathrm{H}$ NMR $(300$ $\left.\mathrm{MHz}, \mathrm{CDCl}_{3}\right) \delta 7.21(\mathrm{~d}, J=4.8 \mathrm{~Hz}, 1 \mathrm{H}), 6.97(\mathrm{~d}, J=3.3 \mathrm{~Hz}, 1 \mathrm{H}), 6.92(\mathrm{dd}, J=4.8,3.3 \mathrm{~Hz}, 1 \mathrm{H})$, $6.03(\mathrm{ddd}, J=17.4,10.5,9.0 \mathrm{~Hz}, 1 \mathrm{H}), 5.25(\mathrm{~d}, J=17.4 \mathrm{~Hz}, 1 \mathrm{H}), 5.19$ (d, $J=10.5 \mathrm{~Hz}, 1 \mathrm{H}), 4.46$ $(\mathrm{s}, 1 \mathrm{H}), 3.82(\mathrm{~d}, J=9.0 \mathrm{~Hz}, 1 \mathrm{H}), 1.38(\mathrm{~s}, 9 \mathrm{H}), 1.28(\mathrm{~s}, 9 \mathrm{H}) ;{ }^{13} \mathrm{C} \mathrm{NMR}\left(75 \mathrm{MHz}, \mathrm{CDCl}_{3}\right) \delta 158.0$, 147.2, 139.5, 126.2, 125.0, 124.6, 116.8, 65.3, 59.4, 53.7, 53.6, 28.8; HRMS Calcd for $\mathrm{C}_{17} \mathrm{H}_{27} \mathrm{~N}_{2} \mathrm{OS}(\mathrm{M}+1)$ : 307.1839. Found: 307.1830. 


\section{Table 1, Entry 13}

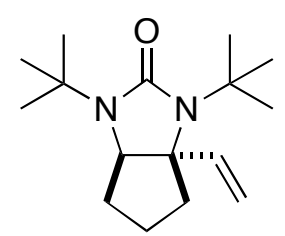

Colorless oil; $[\alpha]^{20}{ }_{\mathrm{D}}=-18.4\left(c 0.64, \mathrm{CHCl}_{3}\right)\left(87 \%\right.$ ee); IR (film) $1682 \mathrm{~cm}^{-1} ;{ }^{1} \mathrm{H}$ NMR $(300$ $\left.\mathrm{MHz}, \mathrm{CDCl}_{3}\right) \delta 6.01(\mathrm{dd}, J=17.4,10.5 \mathrm{~Hz}, 1 \mathrm{H}), 5.12(\mathrm{~d}, J=17.4 \mathrm{~Hz}, 1 \mathrm{H}), 5.04(\mathrm{~d}, J=10.5 \mathrm{~Hz}$, $1 \mathrm{H}), 3.36(\mathrm{dd}, J=6.9,6.3 \mathrm{~Hz}, 1 \mathrm{H}), 2.32-2.20(\mathrm{~m}, 1 \mathrm{H}), 2.14-2.02(\mathrm{~m}, 1 \mathrm{H}), 1.96-1.82(\mathrm{~m}, 1 \mathrm{H})$, 1.81-1.64 (m, 1H), 1.63-1.46 (m, 1H), 1.44-1.32 (m, 1H), $1.41(\mathrm{~s}, 9 \mathrm{H}), 1.36(\mathrm{~s}, 9 \mathrm{H}) ;{ }^{13} \mathrm{C}$ NMR $\left(75 \mathrm{MHz}, \mathrm{CDCl}_{3}\right) \delta$ 158.9, 145.5, 111.9, 69.4, 67.0, 54.8, 53.2, 39.2, 37.5, 29.2, 28.9, 24.4.

Du, H.; Zhao, B.; Shi, Y. J. Am. Chem. Soc., 2007, 129, 762.

\section{Table 1, Entry 14}

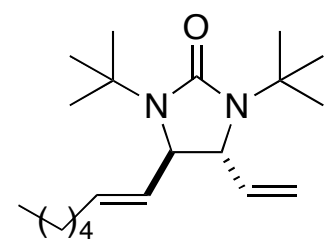

Colorless oil; $[\alpha]^{20}{ }_{\mathrm{D}}=+60.4\left(c 0.52, \mathrm{CHCl}_{3}\right)(92 \%$ ee $) ;$ IR (film) $1692 \mathrm{~cm}^{-1} ;{ }^{1} \mathrm{H}$ NMR $(300$ $\left.\mathrm{MHz}, \mathrm{CDCl}_{3}\right) \delta 5.96(\mathrm{ddd}, J=17.7,10.5,8.4 \mathrm{~Hz}, 1 \mathrm{H}), 5.70-5.50(\mathrm{~m}, 2 \mathrm{H}), 5.20(\mathrm{~d}, J=17.7 \mathrm{~Hz}$, $1 \mathrm{H}), 5.10(\mathrm{~d}, J=10.5 \mathrm{~Hz}, 1 \mathrm{H}), 3.58(\mathrm{~d}, J=8.1 \mathrm{~Hz}, 1 \mathrm{H}), 3.56(\mathrm{~d}, J=7.5 \mathrm{~Hz}, 1 \mathrm{H}), 2.10-1.98(\mathrm{~m}$, 2H), 1.50-1.10 (m, 6H), $1.34(\mathrm{~s}, 9 \mathrm{H}), 1.33(\mathrm{~s}, 9 \mathrm{H}), 0.88(\mathrm{t}, J=6.6 \mathrm{~Hz}, 3 \mathrm{H}) ;{ }^{13} \mathrm{C} \mathrm{NMR}(75 \mathrm{MHz}$, $\left.\mathrm{CDCl}_{3}\right) \delta 158.4,139.8,132.6,131.0,115.8,63.3,62.4,53.3,53.2,32.2,31.5,28.9,22.6,14.2$.

Du, H.; Zhao, B.; Shi, Y. J. Am. Chem. Soc., 2007, 129, 762. 


\section{Scheme 3}

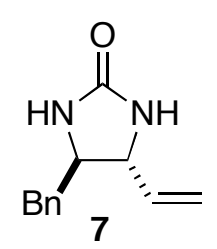

White solid; $\operatorname{mp~} 103-104{ }^{\circ} \mathrm{C}$ (lit. $101-102{ }^{\circ} \mathrm{C}$ ); $[\alpha]_{\mathrm{D}}^{20}=+46.2$ (c 1.09, toluene) (92\% ee) (lit. $[\alpha]_{\mathrm{D}}^{22}=+42.8, c$ 1.09, toluene); IR (film) 3230, $1705 \mathrm{~cm}^{-1} ;{ }^{1} \mathrm{H}$ NMR $\left(300 \mathrm{MHz}, \mathrm{CDCl}_{3}\right) \delta 7.36-$ $7.22(\mathrm{~m}, 3 \mathrm{H}), 7.21-7.15(\mathrm{~m}, 2 \mathrm{H}), 5.80(\mathrm{ddd}, J=17.4,10.5,7.5 \mathrm{~Hz}, 1 \mathrm{H}), 5.24(\mathrm{~d}, J=17.4 \mathrm{~Hz}$, $1 \mathrm{H}), 5.18(\mathrm{~d}, J=10.5 \mathrm{~Hz}, 1 \mathrm{H}), 4.65(\mathrm{~s}, 1 \mathrm{H}), 4.48(\mathrm{~s}, 1 \mathrm{H}), 3.95(\mathrm{dd}, J=7.5,6.6 \mathrm{~Hz}, 1 \mathrm{H}), 3.68-$ $3.58(\mathrm{~m}, 1 \mathrm{H}), 2.93(\mathrm{dd}, J=13.2,4.8 \mathrm{~Hz}, 1 \mathrm{H}), 2.77(\mathrm{dd}, J=13.2,9.0 \mathrm{~Hz}, 1 \mathrm{H}) ;{ }^{13} \mathrm{C}$ NMR $(75$ $\left.\mathrm{MHz}, \mathrm{CDCl}_{3}\right) \delta 162.8,137.2,129.3,128.9,127.0,117.3,61.1,60.5$, 41.1; HRMS Calcd for $\mathrm{C}_{12} \mathrm{H}_{15} \mathrm{~N}_{2} \mathrm{O}(\mathrm{M}+1):$ 203.1179. Found: 203.1177.

Oshitari, T.; Akagi, R.; Mandai, T. Synthesis, 2004, 1325.

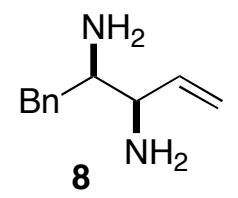

Dark yellow oil; $[\alpha]^{20}{ }_{\mathrm{D}}=+33.3\left(c 1.04, \mathrm{CHCl}_{3}\right)\left(>98 \%\right.$ ee); IR (film) 3362, $3288 \mathrm{~cm}^{-1} ;{ }^{1} \mathrm{H}$ NMR (300 MHz, $\left.\mathrm{CDCl}_{3}\right) \delta$ 7.38-7.16 (m, 5H), $5.90(\mathrm{ddd}, J=17.1,10.2,6.0 \mathrm{~Hz}, 1 \mathrm{H}), 5.24(\mathrm{~d}, J$ $=14.1 \mathrm{~Hz}, 1 \mathrm{H}), 5.17(\mathrm{~d}, J=10.2 \mathrm{~Hz}, 1 \mathrm{H}), 3.24(\mathrm{dd}, J=6.0,5.1 \mathrm{~Hz}, 1 \mathrm{H}), 3.02-2.86(\mathrm{~m}, 2 \mathrm{H}), 2.48$ $(\mathrm{dd}, J=17.1,10.5 \mathrm{~Hz}, 1 \mathrm{H}), 1.28(\mathrm{~s}, 4 \mathrm{H}) ;{ }^{13} \mathrm{C} \mathrm{NMR}\left(75 \mathrm{MHz}, \mathrm{CDCl}_{3}\right) \delta 141.2,139.5,129.1$, 128.4, 126.2, 115.1, 58.4, 56.8, 40.9; HRMS Calcd for $\mathrm{C}_{11} \mathrm{H}_{17} \mathrm{~N}_{2}(\mathrm{M}+1)$ : 177.1392. Found: 177.1392 .

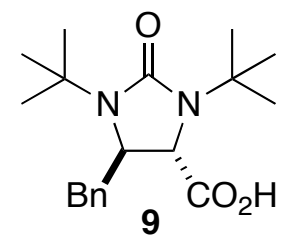


White solid; mp 181-182 ${ }^{\circ} \mathrm{C}$; $[\alpha]^{20}{ }_{\mathrm{D}}=-46.2\left(c 0.58, \mathrm{CHCl}_{3}, 92 \%\right.$ ee); IR (film) 1746,1622 $\mathrm{cm}^{-1} ;{ }^{1} \mathrm{H}$ NMR $\left(300 \mathrm{MHz}, \mathrm{CDCl}_{3}\right) \delta$ 7.39-7.24 (m, 3H), $7.19(\mathrm{~d}, J=6.6 \mathrm{~Hz}, 2 \mathrm{H}), 3.89(\mathrm{dd}, J=$ 9.3, $3.3 \mathrm{~Hz}, 1 \mathrm{H}), 3.83$ (s, 1H), 3.08 (dd, $J=13.8,3.3 \mathrm{~Hz}, 1 \mathrm{H}), 2,68(\mathrm{dd}, J=13.8 .9 .3 \mathrm{~Hz}, 1 \mathrm{H})$, $1.45(\mathrm{~s}, 9 \mathrm{H}), 1.28(\mathrm{~s}, 9 \mathrm{H}) ;{ }^{13} \mathrm{C} \mathrm{NMR}\left(75 \mathrm{MHz}, \mathrm{CDCl}_{3}\right) \delta 176.8,158.4,136.3,129.4,129.0$, 127.2, 58.2, 57.9, 53.5, 53.4, 41.0, 29.1, 28.5; HRMS Calcd for $\mathrm{C}_{19} \mathrm{H}_{29} \mathrm{~N}_{2} \mathrm{O}_{3}(\mathrm{M}+1)$ : 333.2173; Found: 333.2166 .

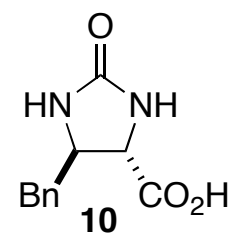

White solid; mp 203-204 ${ }^{\circ} \mathrm{C}$ (lit. mp $\left.210{ }^{\circ} \mathrm{C}\right) ;[\alpha]^{20}{ }_{\mathrm{D}}=+71.1(c 0.47, \mathrm{MeOH}) ;{ }^{1} \mathrm{H}$ NMR $(300$ $\left.\mathrm{MHz}, \mathrm{CD}_{3} \mathrm{OD}\right) \delta$ 7.36-7.20 (m, 5H), 4.07 (ddd, $\left.J=6.6,5.7,4.2 \mathrm{~Hz}, 1 \mathrm{H}\right), 3.99(\mathrm{~d}, J=4.2 \mathrm{~Hz}$, $1 \mathrm{H}), 2.97(\mathrm{dd}, J=13.6,5.7 \mathrm{~Hz}, 1 \mathrm{H}), 2.90(\mathrm{dd}, J=13.6,6.6 \mathrm{~Hz}, 1 \mathrm{H}) ;{ }^{13} \mathrm{C}$ NMR $(75 \mathrm{MHz}$, $\left.\mathrm{CD}_{3} \mathrm{OD}\right) \delta 175.0,165.0,138.0,130.8,129.8,128.0,59.5,58.8,42.7$; HRMS Calcd for $\mathrm{C}_{11} \mathrm{H}_{13} \mathrm{~N}_{2} \mathrm{O}_{3}(\mathrm{M}+1)$ : 221.0921; Found: 221.0914.

Oshitari, T.; Akagi, R.; Mandai, T. Synthesis, 2004, 1325.

Dunn, P.J.; Häner, R.; Rapoport, H. J. Org. Chem. 1990, 55, 5017.<smiles>NC(Br)C(N)C(=O)O</smiles>

White solid; mp $170{ }^{\circ} \mathrm{C}$ (decomposed) (lit. mp 206-207 ${ }^{\circ} \mathrm{C}$ ); $[\alpha]^{20}{ }_{\mathrm{D}}=+59.8(c, 1.3, \mathrm{MeOH})[$ lit. $\left.[\alpha]^{20}{ }_{\mathrm{D}}=+18.8,(c, 1.4, \mathrm{MeOH})\right] ;{ }^{1} \mathrm{H} \mathrm{NMR}\left(300 \mathrm{MHz}, \mathrm{CD}_{3} \mathrm{OD}\right) \delta$ 7.50-7.30 (m, 5H), $4.64(\mathrm{~d}, J=$ $3.0 \mathrm{~Hz}, 1 \mathrm{H}), 4.16$ (ddd, $J=11.7,3.3,3.0 \mathrm{~Hz}, 1 \mathrm{H}), 3.37$ (dd, $J=14.1,3.3 \mathrm{~Hz}, 1 \mathrm{H}), 2.74$ (dd, $J=$ 14.1, $11.7 \mathrm{~Hz}, 1 \mathrm{H}) ;{ }^{13} \mathrm{C}$ NMR $\left(75 \mathrm{MHz}, \mathrm{CD}_{3} \mathrm{OD}\right) \delta 168.2,135.3,130.7,130.5,129.2,54.6$, $53.2,34.7$.

Dunn, P.J.; Häner, R.; Rapoport, H. J. Org. Chem. 1990, 55, 5017. 


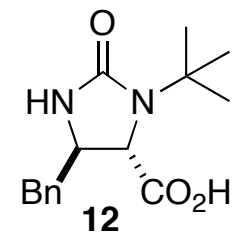

Colorless oil; $[\alpha]^{20}{ }_{\mathrm{D}}=+12.5\left(c 0.725, \mathrm{CHCl}_{3}\right)$; IR (film) $3307,1720,1629 \mathrm{~cm}^{-1} ;{ }^{1} \mathrm{H}$ NMR $(300$ $\mathrm{MHz}, \mathrm{CDCl}_{3}$ ) $\delta 11.72$ (bs, 1H), $7.40-7.26(\mathrm{~m}, 3 \mathrm{H}), 7.20$ (d, J = 6.9 Hz, 2H), 6.89 (bs, 1H), 4.11 (s, 1H), 3.96-3.84 (m, 1H), 2.91 (dd, $J=13.5,4.5 \mathrm{~Hz}, 1 \mathrm{H}), 2.79$ (dd, $J=13.5,5.4 \mathrm{~Hz}, 1 \mathrm{H}) ; 1.21$ (s, 9H); ${ }^{13} \mathrm{C} \mathrm{NMR}\left(75 \mathrm{MHz}, \mathrm{CDCl}_{3}\right) \delta 176.5,162.6,135.2,129.7,129.0,127.5,61.3,55.6,54.4$, 42.0, 28.1; HRMS Calcd for $\mathrm{C}_{19} \mathrm{H}_{21} \mathrm{~N}_{2} \mathrm{O}_{3}(\mathrm{M}+1)$ : 277.1547; Found: 277.1539 . 
The chromatograms for the determination of enantioselectivity

\section{Table 1, Entry 1}<smiles>C=C[C@@H]1NC(=O)N[C@H]1C</smiles>

GC Conditions: Column: Chiraldex B-DM (Cat No. 77023), Advanced Separation Technologies, Inc. Oven: $140^{\circ} \mathrm{C}$; Carrier: Helium, head pressure $30 \mathrm{psi}$; Detection: FID $250^{\circ} \mathrm{C}$

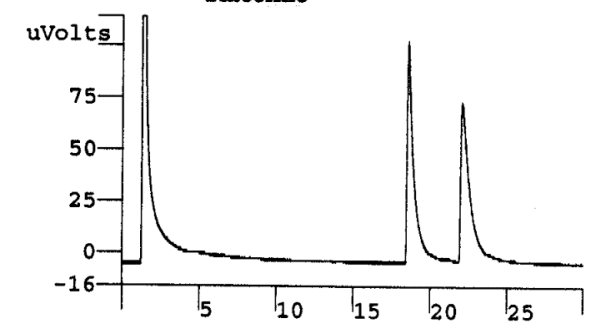

Chiral
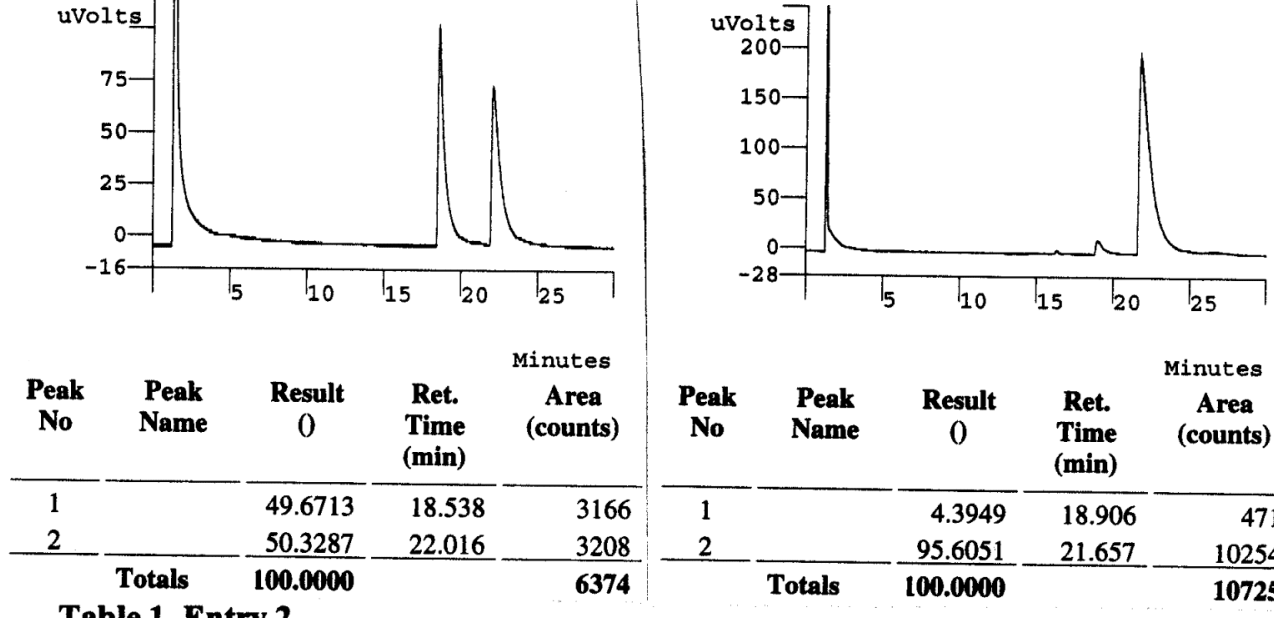

Table 1, Entry 2

\begin{tabular}{|c|c|c|c|c|}
\hline $\begin{array}{c}\text { Peak } \\
\text { No }\end{array}$ & $\begin{array}{c}\text { Peak } \\
\text { Name }\end{array}$ & $\begin{array}{c}\text { Result } \\
0\end{array}$ & $\begin{array}{c}\text { Ret. } \\
\text { Time } \\
\text { (min) }\end{array}$ & $\begin{array}{c}\text { Minutes } \\
\text { Area } \\
\text { (counts) }\end{array}$ \\
\hline 1 & & 4.3949 & 18.906 & 471 \\
\hline 2 & & 95.6051 & 21.657 & 10254 \\
\hline & Totals & 100.0000 & & 10725 \\
\hline
\end{tabular}<smiles>C=C[C@@H]1[C@H](CC)N(C(C)(C)C)C(=O)N1C(C)(C)C</smiles>

GC Conditions: Column: Chiraldex B-DM (Cat No. 77023), Advanced Separation Technologies, Inc..

Oven: $95^{\circ} \mathrm{C}$; Carrier: Helium, head pressure 30 psi; Detection: FID $250{ }^{\circ} \mathrm{C}$

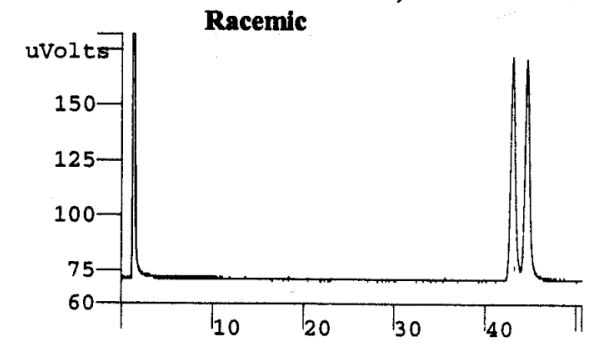

Chiral
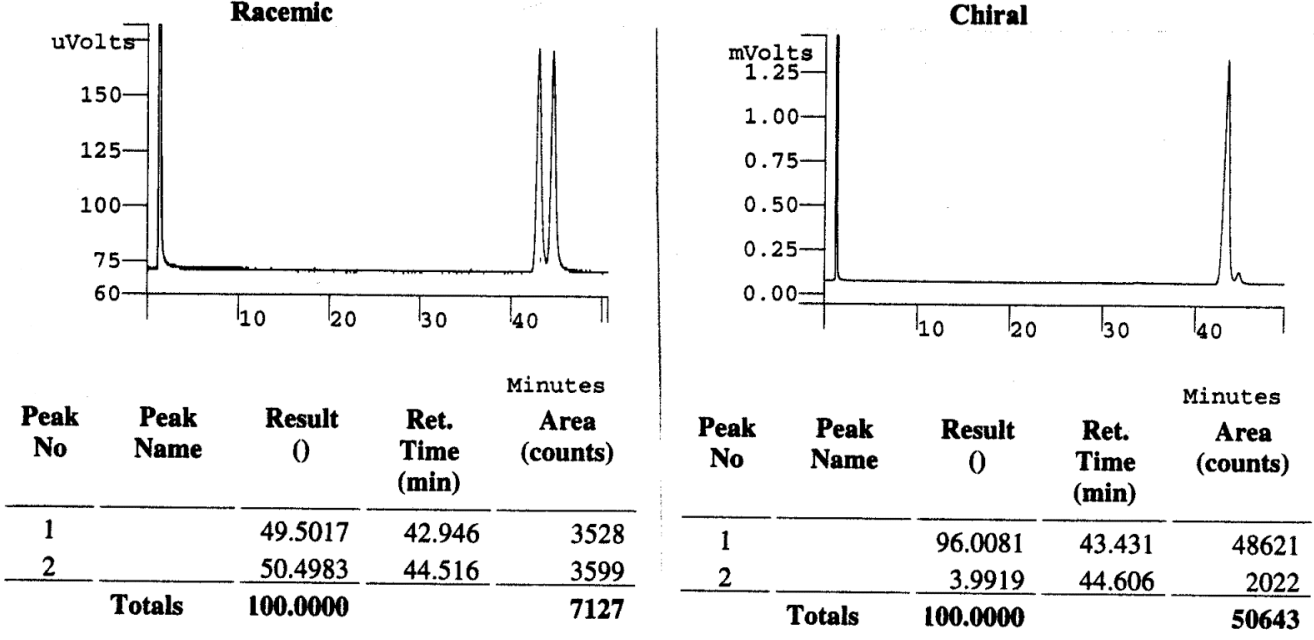
Table 1, Entry 3

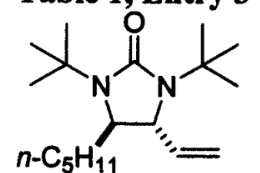

GC Conditions: Column: Chiraldex B-DM (Cat No. 77023), Advanced Separation Technologies, Inc.. Oven: $100^{\circ} \mathrm{C}$; Carrier: Helium, head pressure 30 psi; Detection: FID $250^{\circ} \mathrm{C}$

Racemic
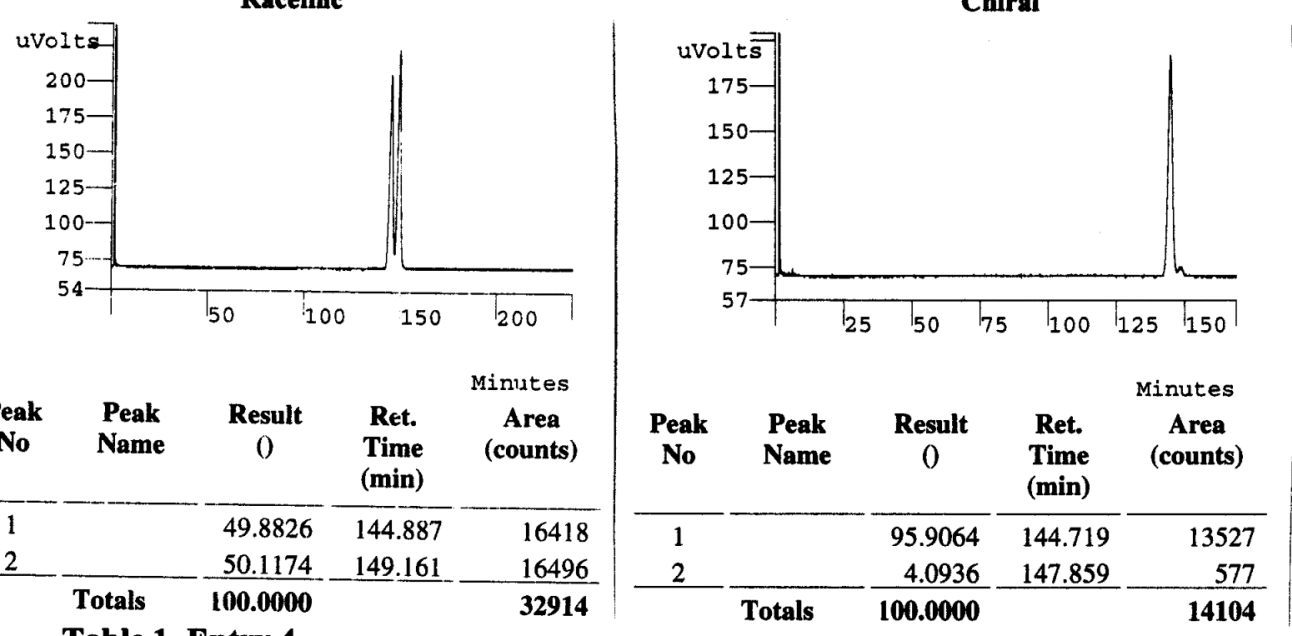

\begin{tabular}{|c|c|c|c|c|}
\hline $\begin{array}{c}\text { Peak } \\
\text { No }\end{array}$ & $\begin{array}{l}\text { Peak } \\
\text { Name }\end{array}$ & $\begin{array}{c}\text { Result } \\
0\end{array}$ & $\begin{array}{l}\text { Ret. } \\
\text { Time } \\
\text { (min) }\end{array}$ & $\begin{array}{c}\text { Minutes } \\
\text { Area } \\
\text { (counts) }\end{array}$ \\
\hline 1 & & 49.8826 & 144.887 & 16418 \\
\hline 2 & & 50.1174 & 149.161 & 16496 \\
\hline & $\begin{array}{l}\text { Totals } \\
\text { Table } 1 \text {, }\end{array}$ & $\begin{array}{l}100.0000 \\
\text { ntry } 4\end{array}$ & & 32914 \\
\hline
\end{tabular}<smiles>C=C[C@@H]1[C@H](Cc2ccccc2)N(C(C)(C)C)C(=O)N1C(C)(C)C</smiles>

HPLC Conditions: Column: Chiralpak AD-H (Column No. ADHOCE-FD069), Daicel Chemical Industries, Ltd.. Eluent: Hexanes/IPA (97/3); Flow rate: $1.0 \mathrm{~mL} / \mathrm{min}$; Detection: UV220
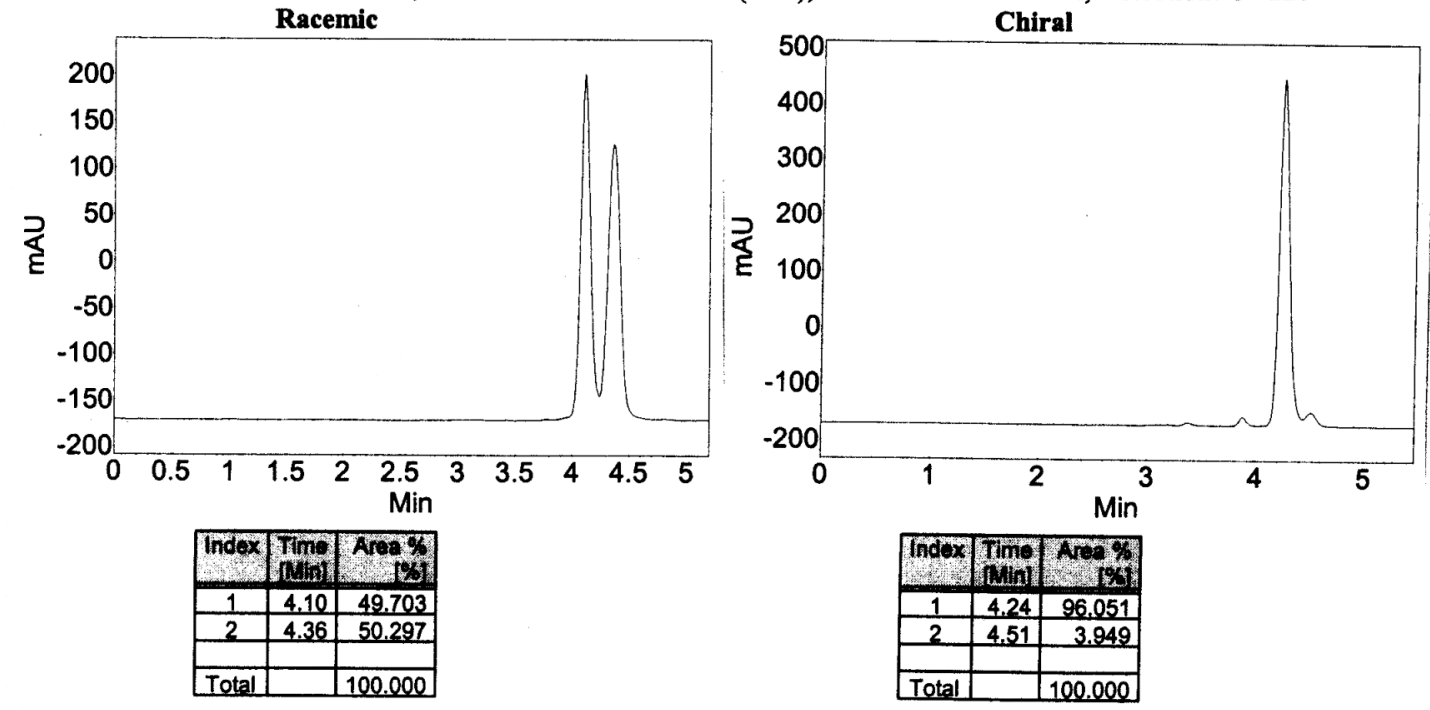
Table 1, Entry 5<smiles>C=C[C@H]1C(CCc2ccccc2)N(C(C)(C)C)C(=O)N1C(C)(C)C</smiles>

HPLC Conditions: Column: Chiralpak AD-H (Column No. ADH0CE-FD069), Daicel Chemical Industries, Ltd.. Eluent: Hexanes/IPA (98/2); Flow rate: $1.0 \mathrm{~mL} / \mathrm{min}$; Detection: UV210

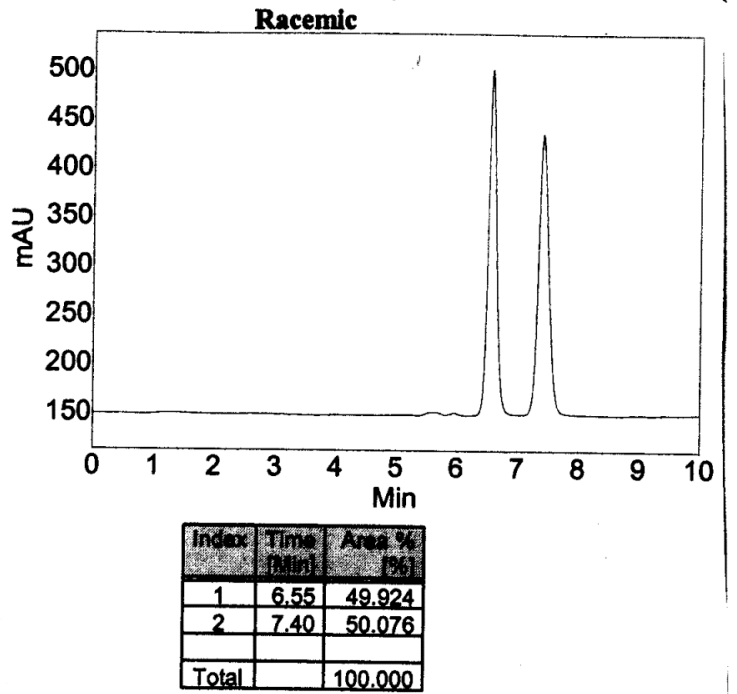

Chiral

Table 1, Entry 6

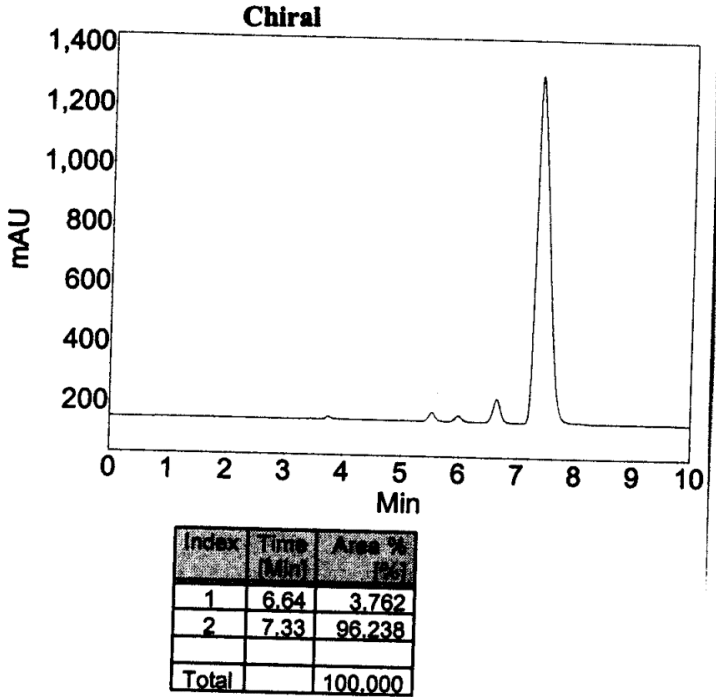<smiles>C=C[C@H]1NC(=O)NC1C1CCCCC1</smiles>

GC Conditions: Column: Chiraldex B-DM (Cat No. 77023), Advanced Separation Technologies, Inc. Oven: $160^{\circ} \mathrm{C}$; Carrier: Helium, head pressure $30 \mathrm{psi}$; Detection: FID $250^{\circ} \mathrm{C}$

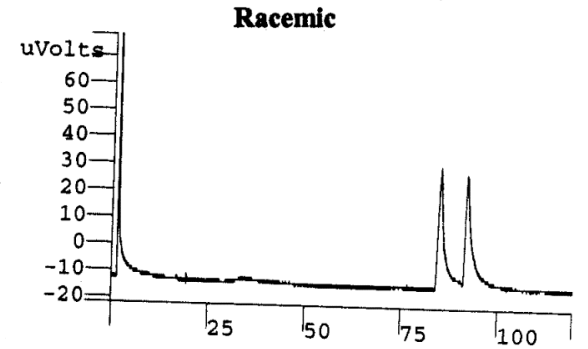

Chiral

\begin{tabular}{ccccc}
$\begin{array}{c}\text { Peak } \\
\text { No }\end{array}$ & $\begin{array}{c}\text { Peak } \\
\text { Name }\end{array}$ & $\begin{array}{c}\text { Result } \\
()\end{array}$ & $\begin{array}{c}\text { Ret. } \\
\text { Time } \\
\text { (min) }\end{array}$ & $\begin{array}{c}\text { Minutes } \\
\text { Area } \\
\text { (counts) }\end{array}$ \\
\hline 1 & & 50.2217 & 84.894 & 4960 \\
\hline 2 & $\frac{49.7783}{\text { Totals }}$ & $\frac{91.647}{100.0000}$ & & 4916 \\
\hline
\end{tabular}

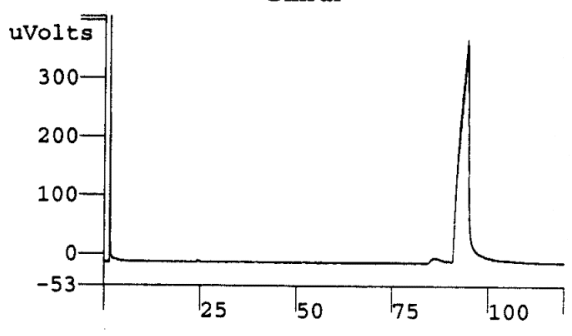

\begin{tabular}{|c|c|c|c|c|}
\hline $\begin{array}{c}\text { Peak } \\
\text { No }\end{array}$ & $\begin{array}{c}\text { Peak } \\
\text { Name }\end{array}$ & $\begin{array}{c}\text { Result } \\
0\end{array}$ & $\begin{array}{l}\text { Ret. } \\
\text { Time } \\
\text { (min) }\end{array}$ & $\begin{array}{c}\text { Minutes } \\
\text { Area } \\
\text { (counts) }\end{array}$ \\
\hline 1 & & 2.3696 & 86.279 & 1555 \\
\hline 2 & & 97.6304 & 94.311 & 64082 \\
\hline & Totals & 100.0000 & & 65637 \\
\hline
\end{tabular}


Table 1, Entry 7<smiles>C=C[C@@H]1[C@H](/C=C\CC)N(C(C)(C)C)C(=O)N1C(C)(C)C</smiles>

GC Conditions: Column: Chiraldex B-DM (Cat No. 77023), Advanced Separation Technologies, Inc.. Oven: $100^{\circ} \mathrm{C}$; Carrier: Helium, head pressure $30 \mathrm{psi}$; Detection: FID $250^{\circ} \mathrm{C}$
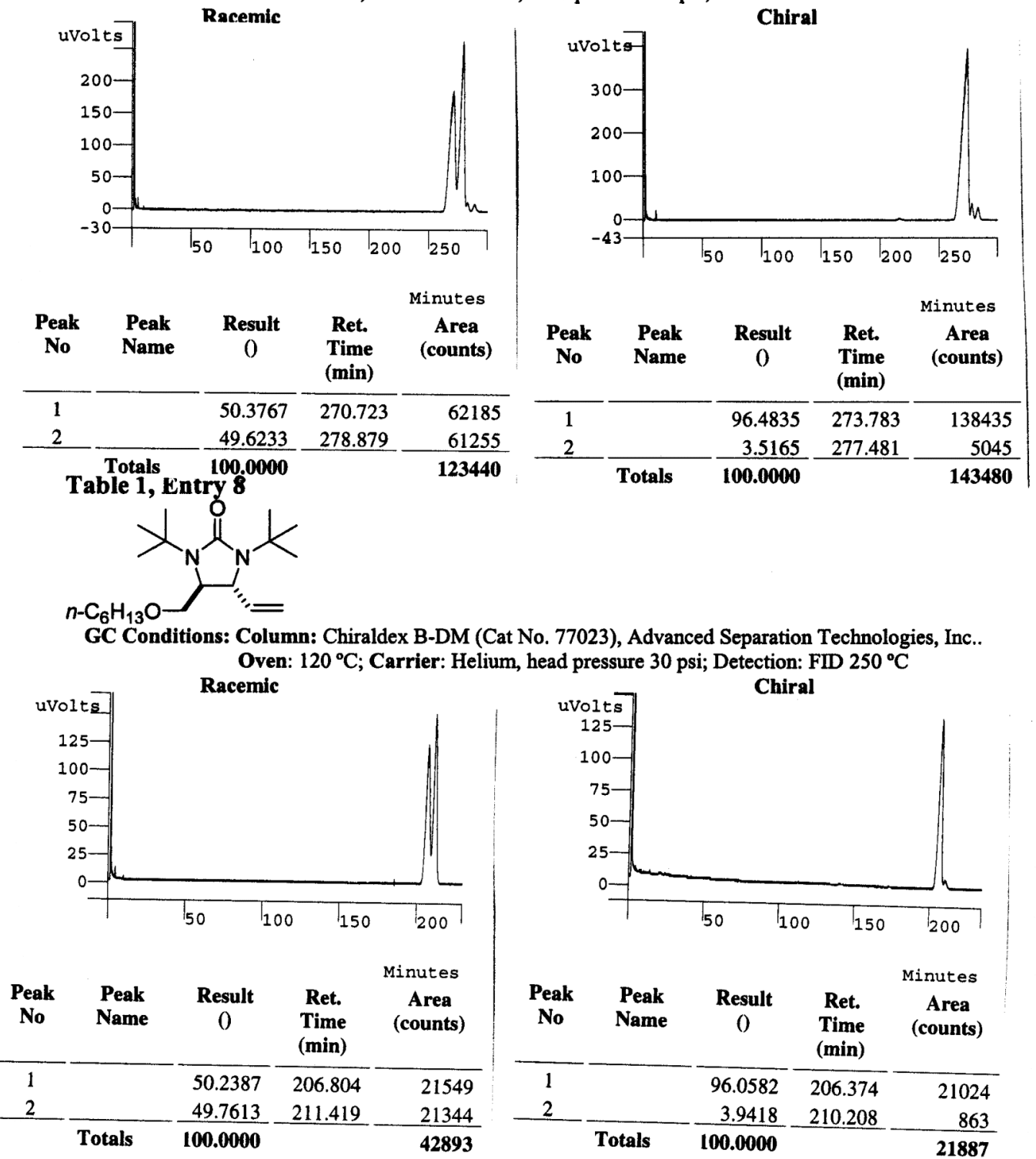
Table 1, Entry 9

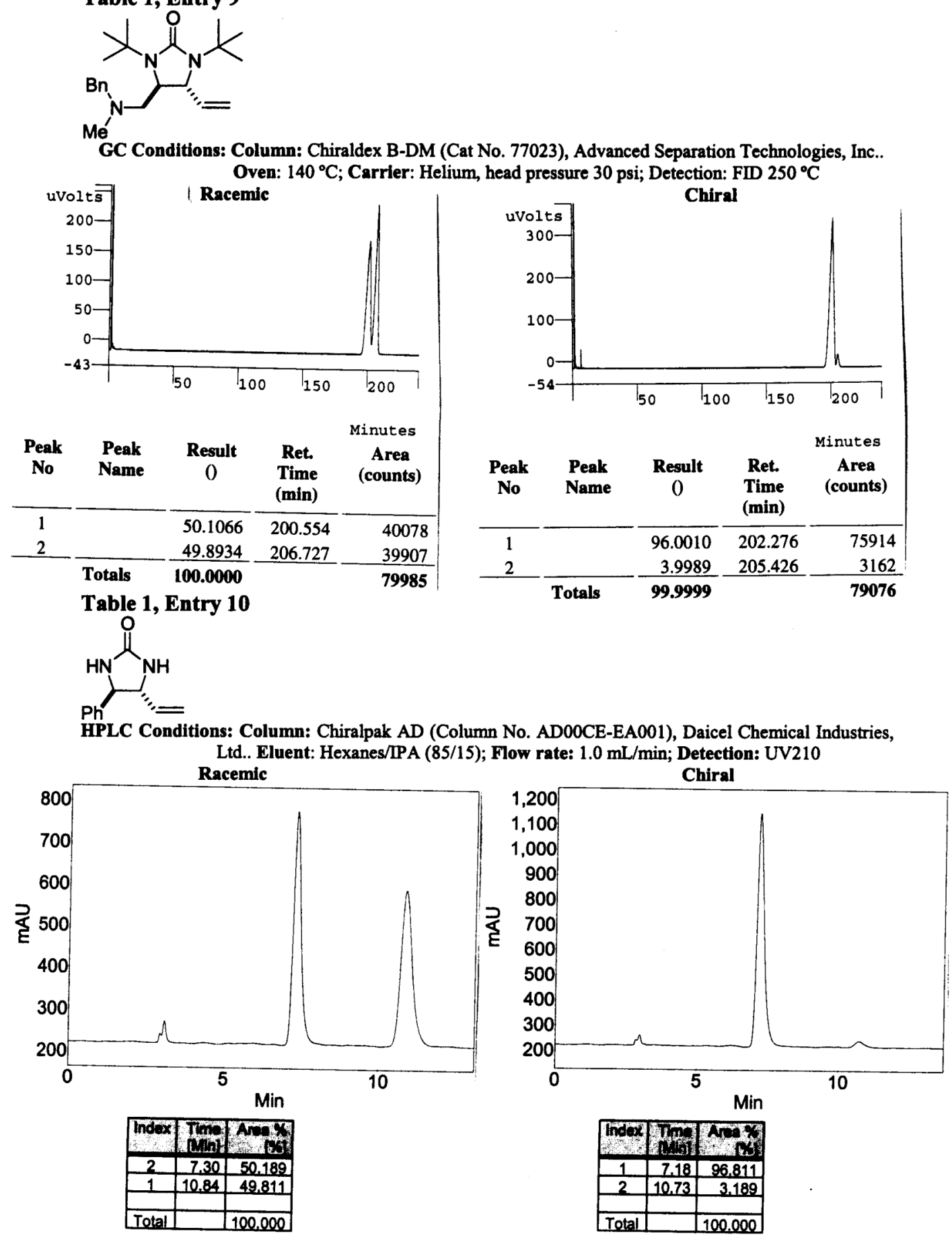


Table 1, Entry 11

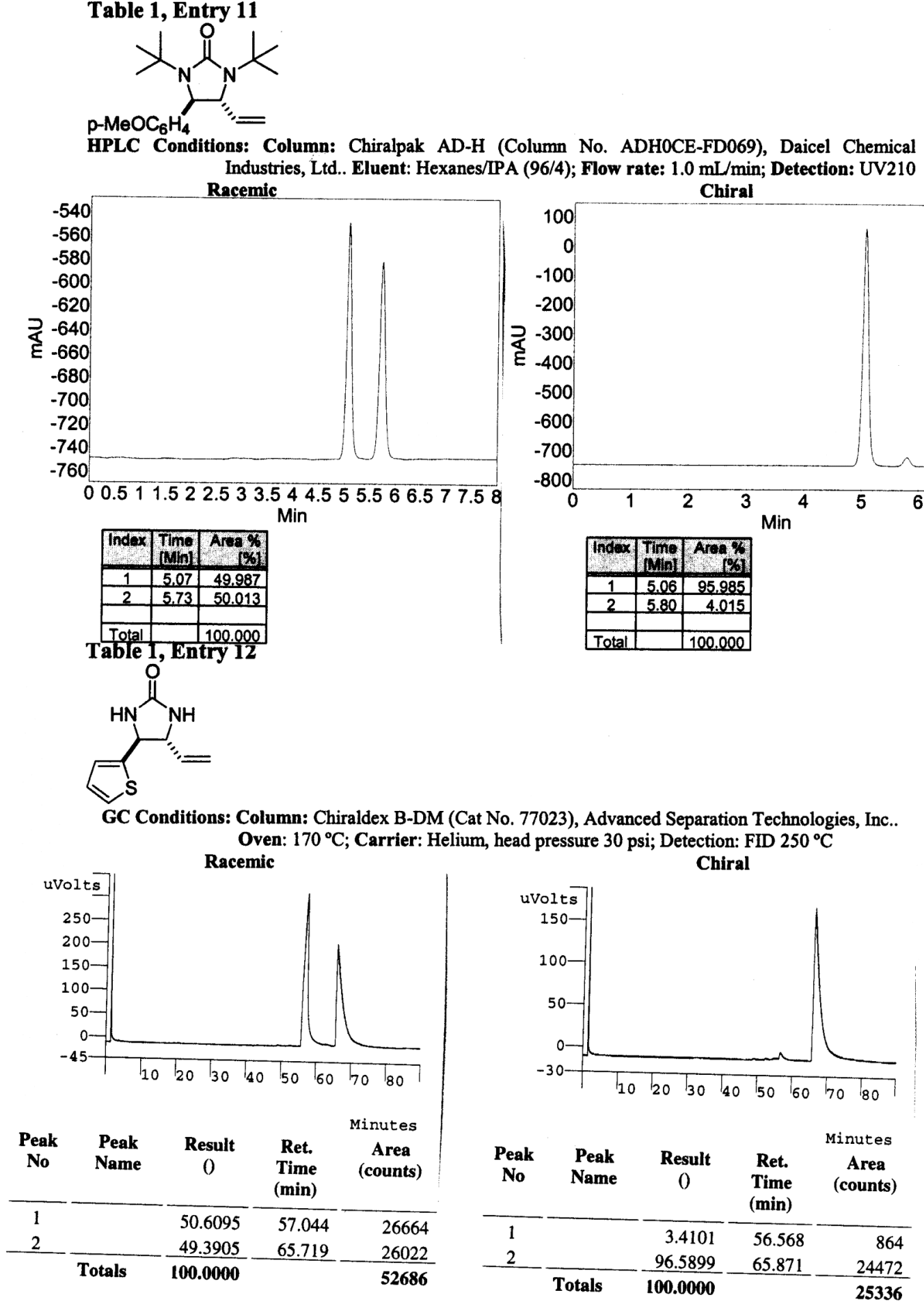




\section{Table 1, Entry 13}

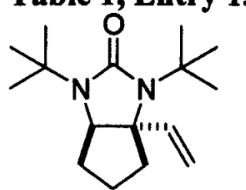

GC Conditions: Column: Chiraldex B-DM (Cat No. 77023), Advanced Separation Technologies, Inc.

Oven: $130^{\circ} \mathrm{C}$; Carrier: Helium, head pressure 30 psi; Detection: FID $250^{\circ} \mathrm{C}$
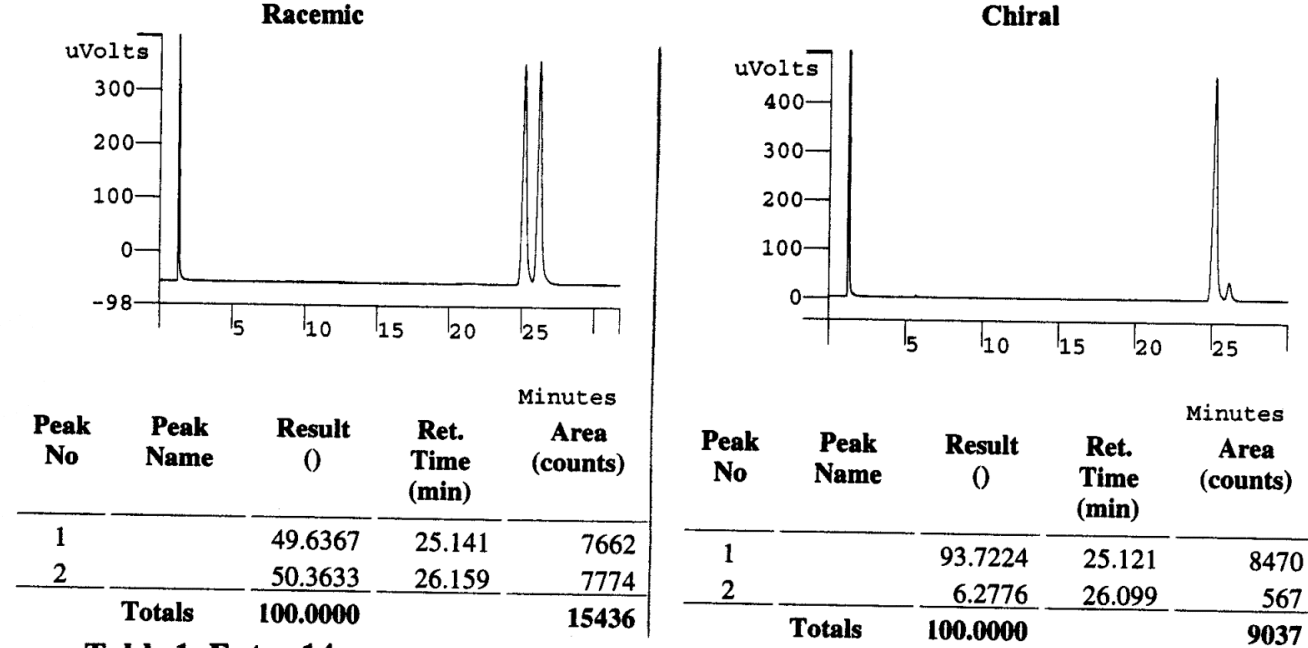

Table 1, Entry 14
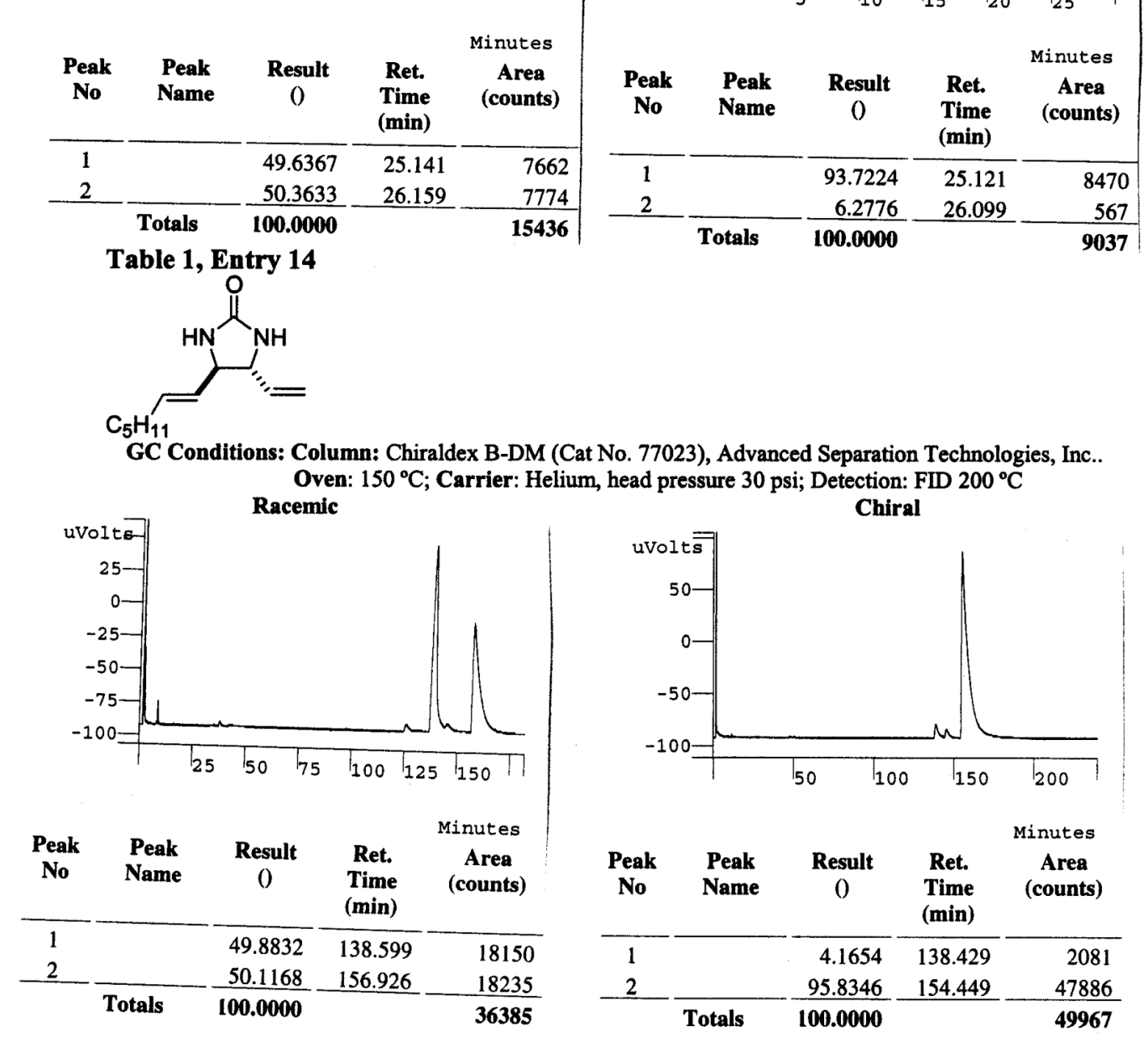

Column: Chiraldex B-DM (Cat No. 77023), Advanced Separation Technologies, Inc.

Oven: $150^{\circ} \mathrm{C}$; Carrier: Helium, head pressure 30 psi; Detection: FID $200^{\circ} \mathrm{C}$

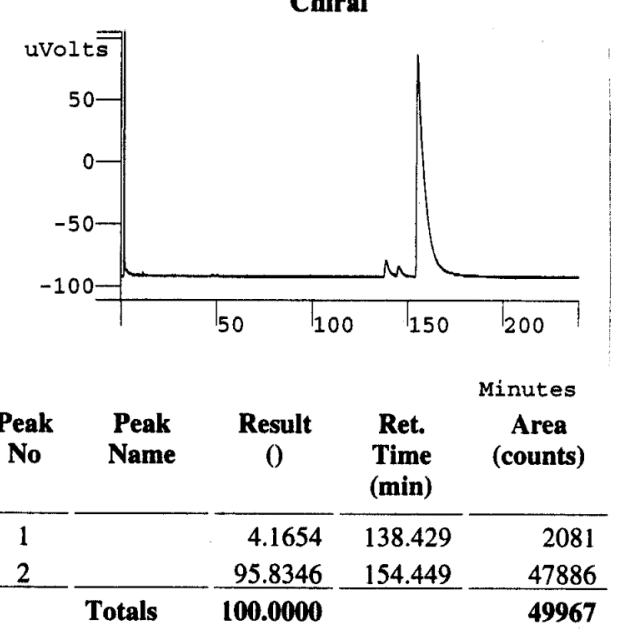


Scheme 3, Compound 7<smiles>CCC[C@H]1C(=O)NC(Br)[C@@H]1C1CCCC1</smiles>

HPLC Conditions: Column: Chiralpak AD (Column No. AD00CE-EA001), Daicel Chemical Industries, Ltd.. Eluent: Hexanes/IPA (90/10); Flow rate: $1.0 \mathrm{~mL} / \mathrm{min}$; Detection: UV210 Racemic

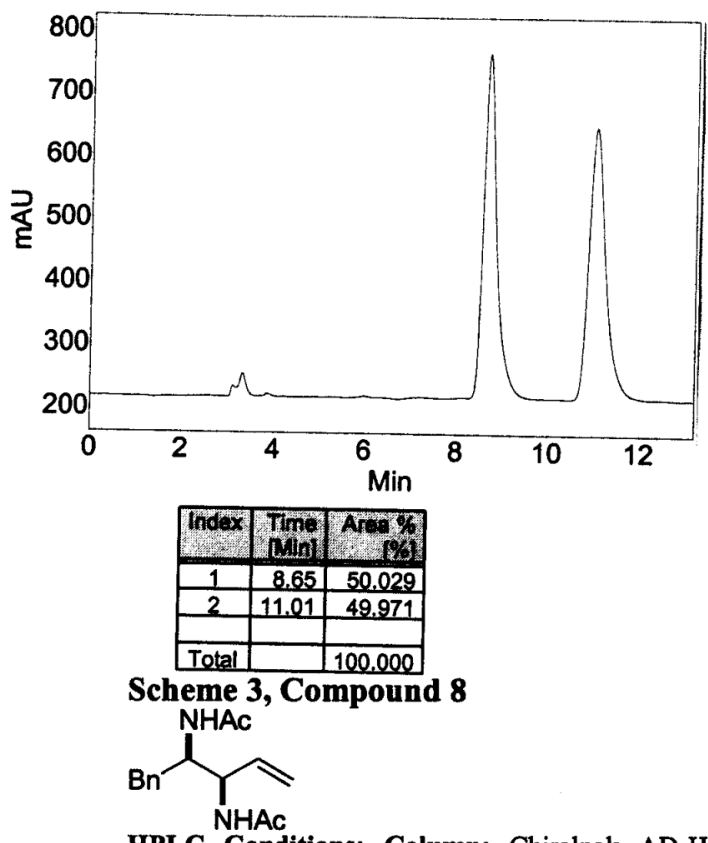

Chiral

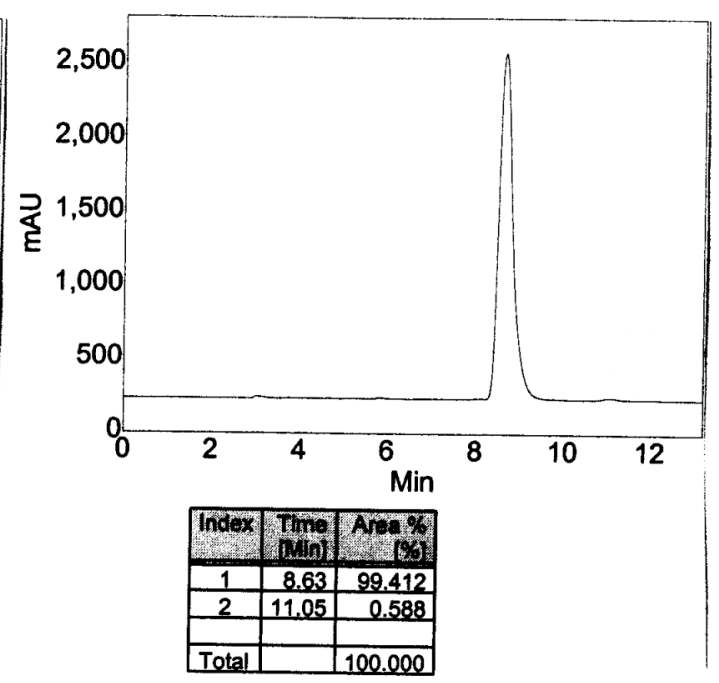

HPLC Conditions: Column: Chiralpak AD-H (Column No. ADH0CE-FD069), Daicel Chemical Industries, Ltd.. Eluent: Hexanes/IPA (98/2); Flow rate: $1.0 \mathrm{~mL} / \mathrm{min}$; Detection: UV210
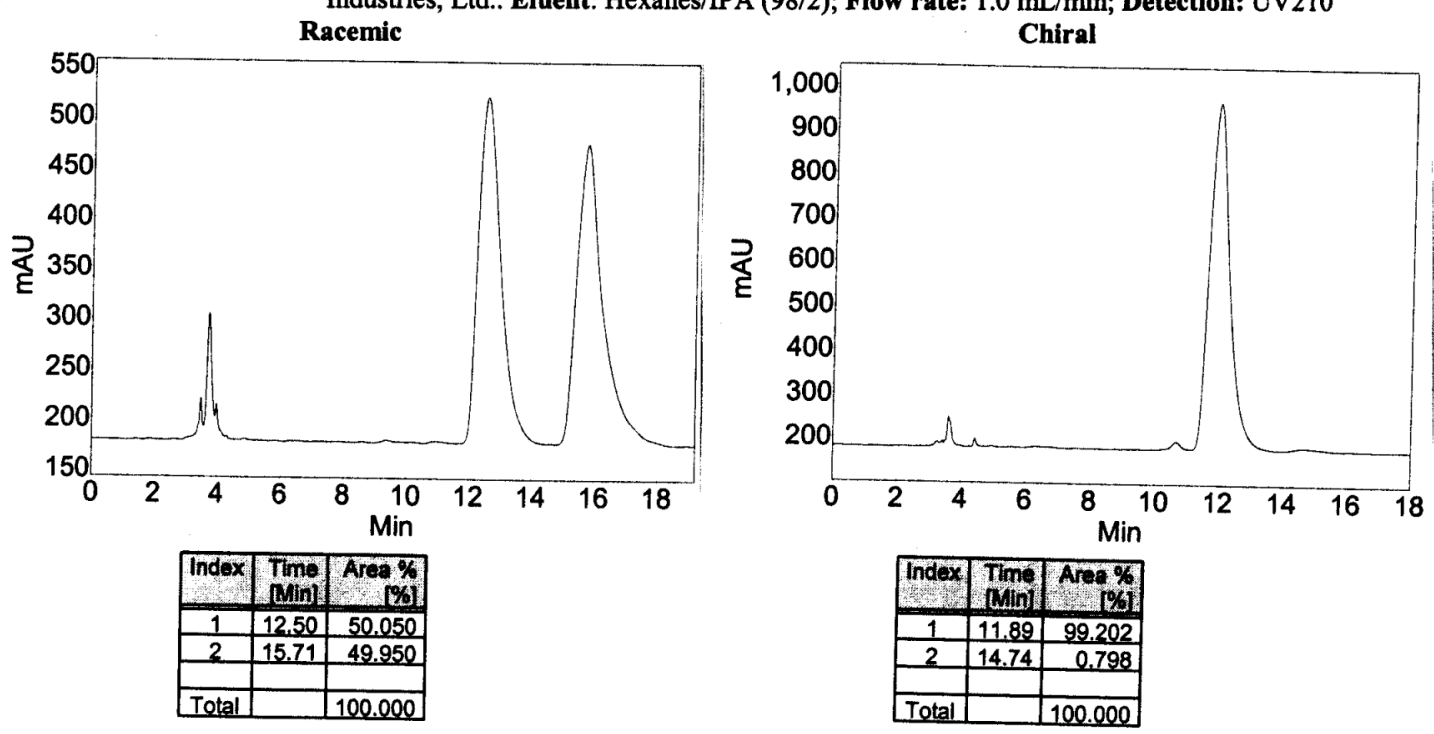


\section{Scheme 3, Compound 10}<smiles>CCOC(=O)NC(Cc1ccccc1)C(C)C</smiles>

HPLC Conditions: Column: Chiralpak AD (Column No. AD00CE-EA001), Daicel Chemical Industries, Ltd. Eluent: Hexanes/IPA (90/10); Flow rate: $1.0 \mathrm{~mL} / \mathrm{min}$; Detection: UV210

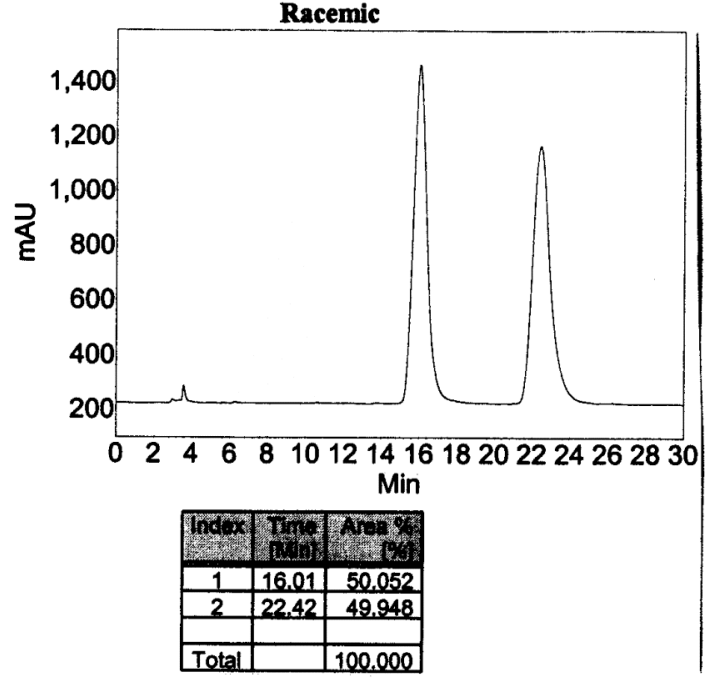

Chiral

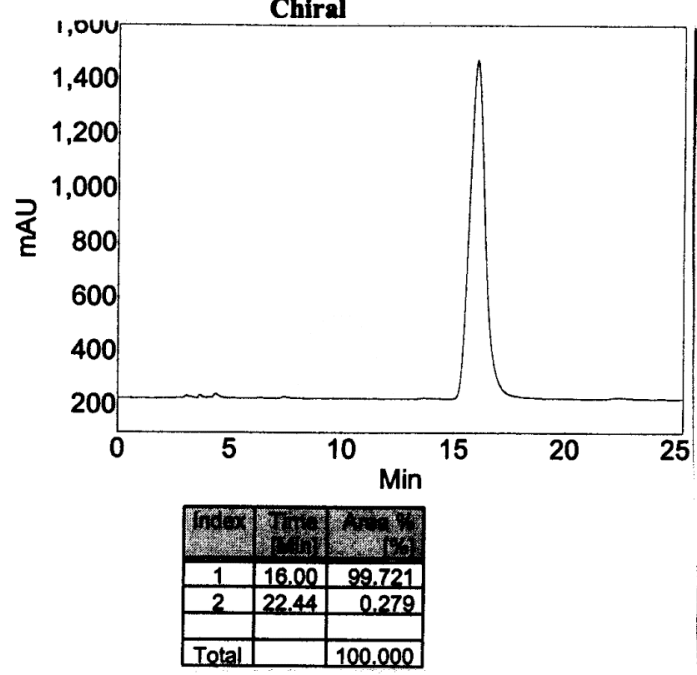

Scheme 3, Compound 12<smiles>COC(=O)[C@H]1C(Cc2ccccc2)NC(=O)N1C(C)(C)C</smiles>

HPLC Conditions: Column: Chiralpak AD (Column No. AD00CE-EA001), Daicel Chemical Industries, Ltd.. Eluent: Hexanes/IPA (90/10); Flow rate: $1.0 \mathrm{~mL} / \mathrm{min}$; Detection: UV220
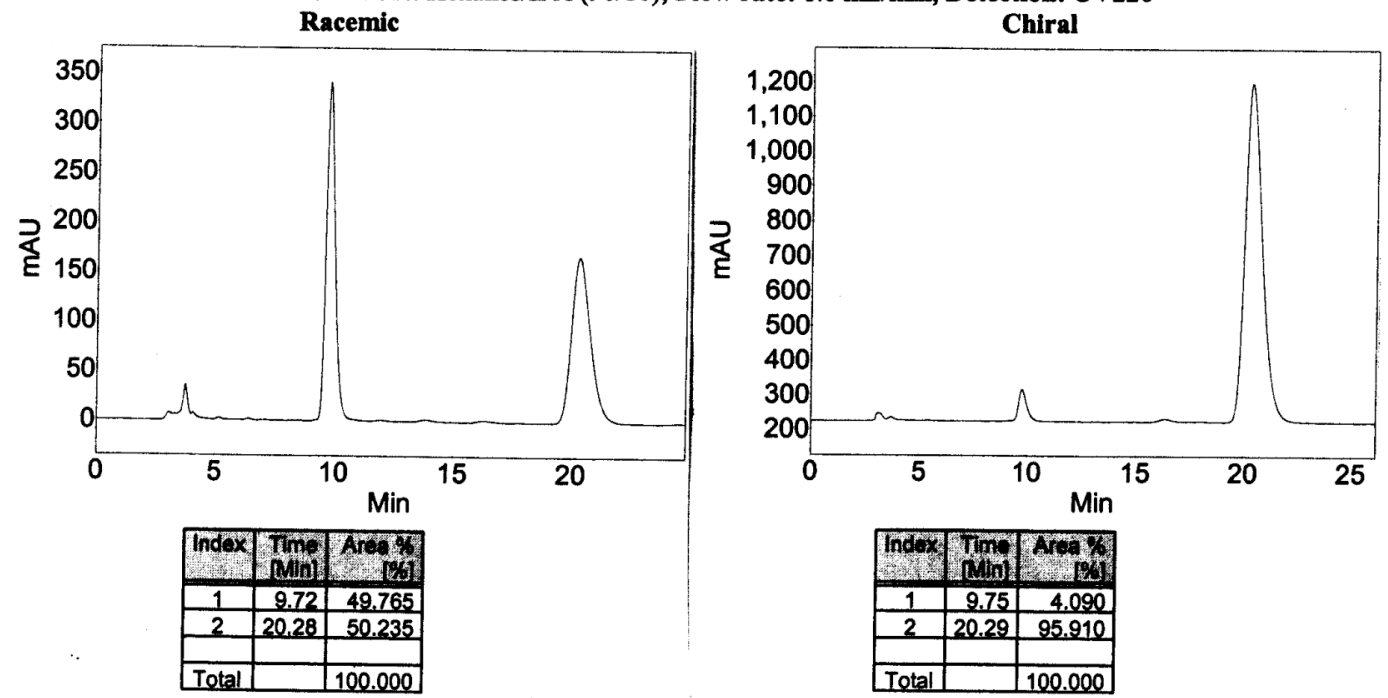


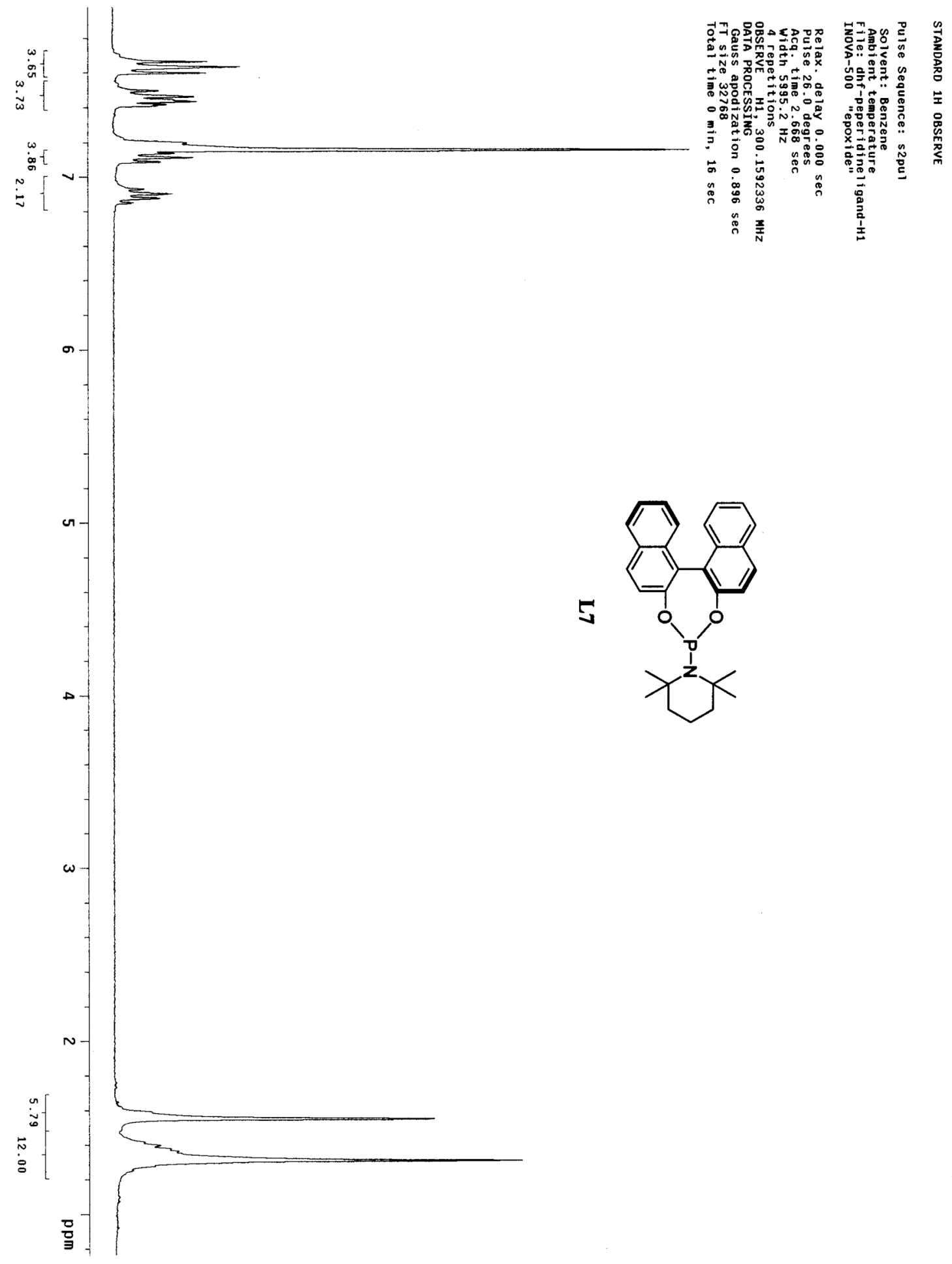



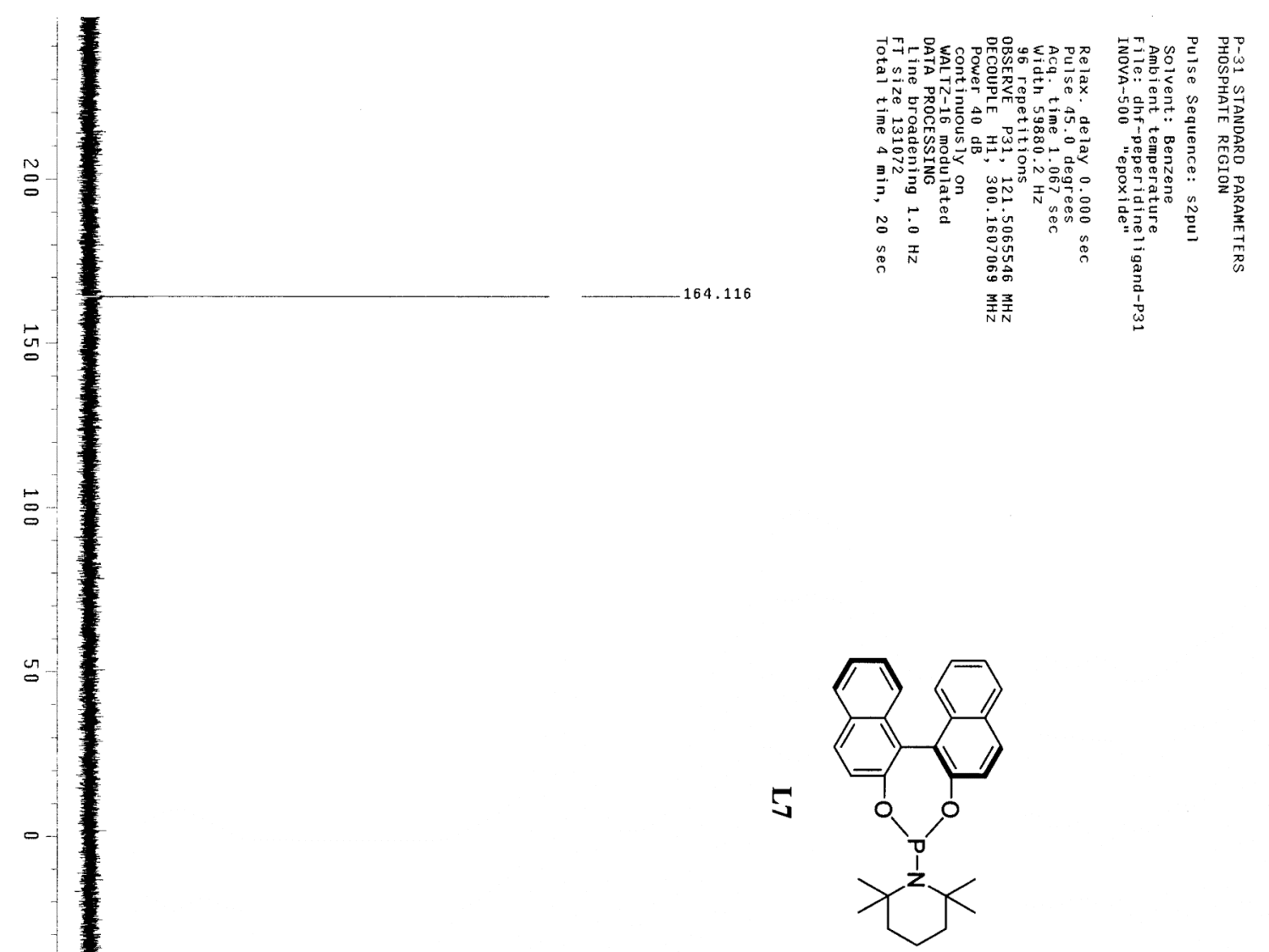


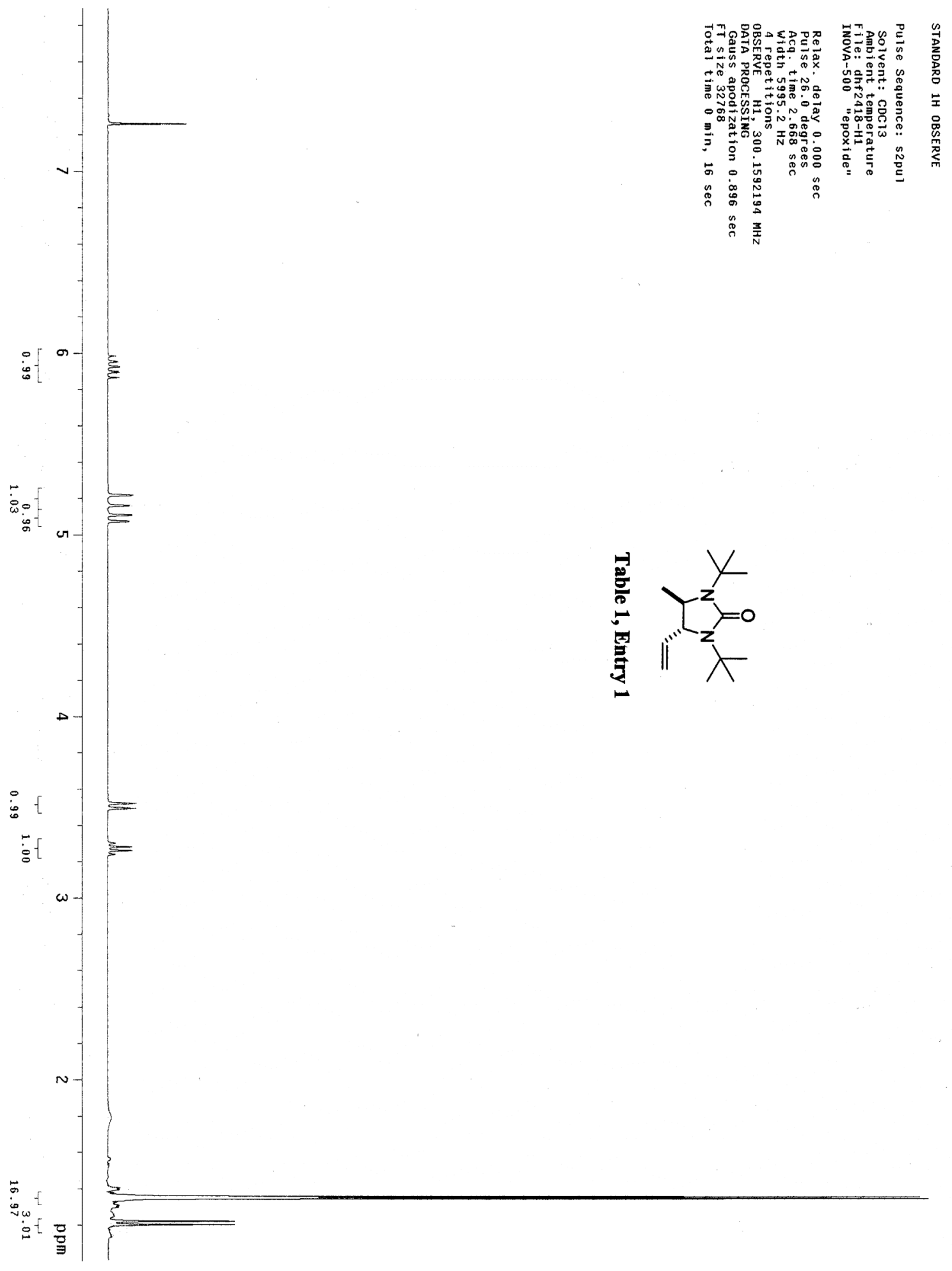




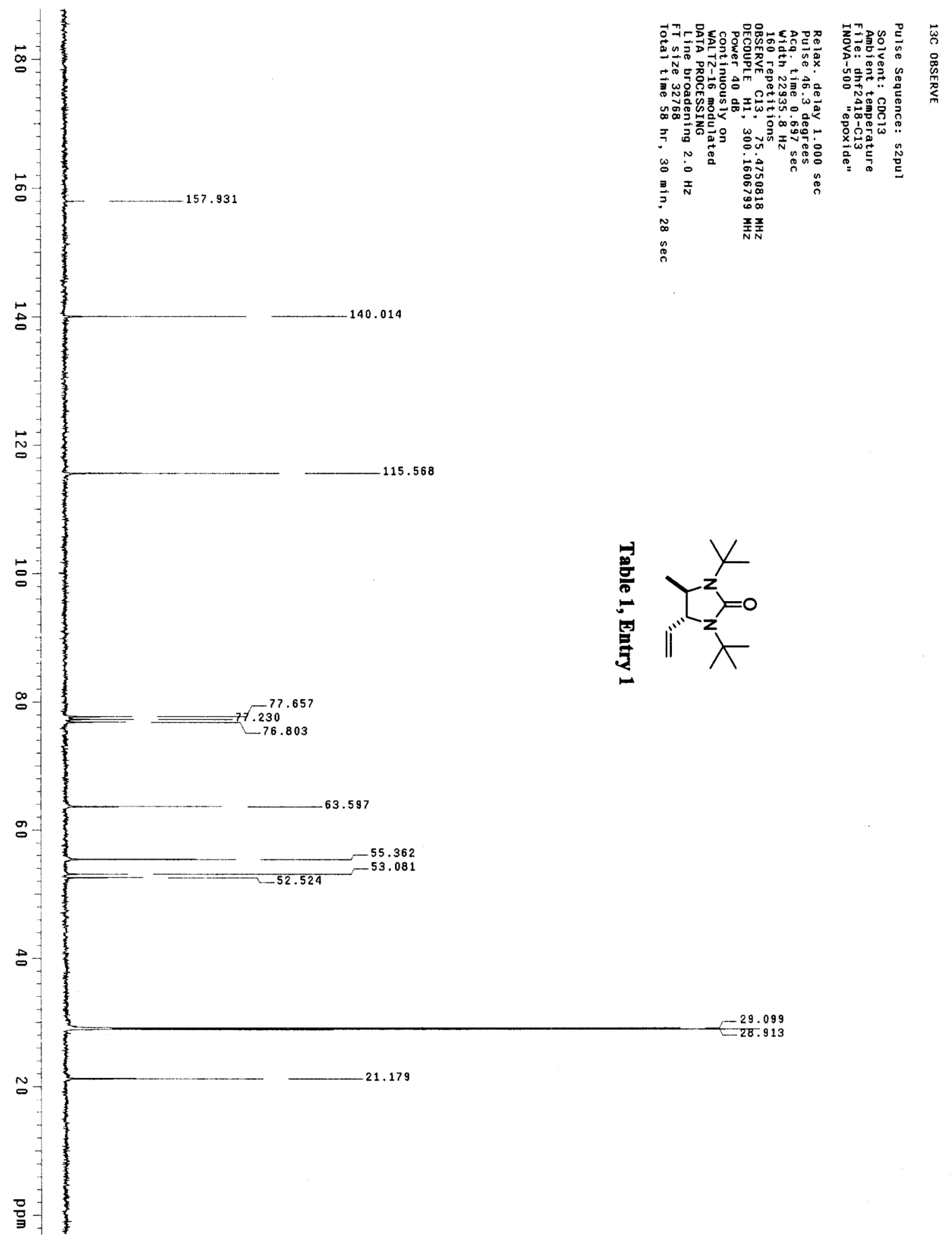




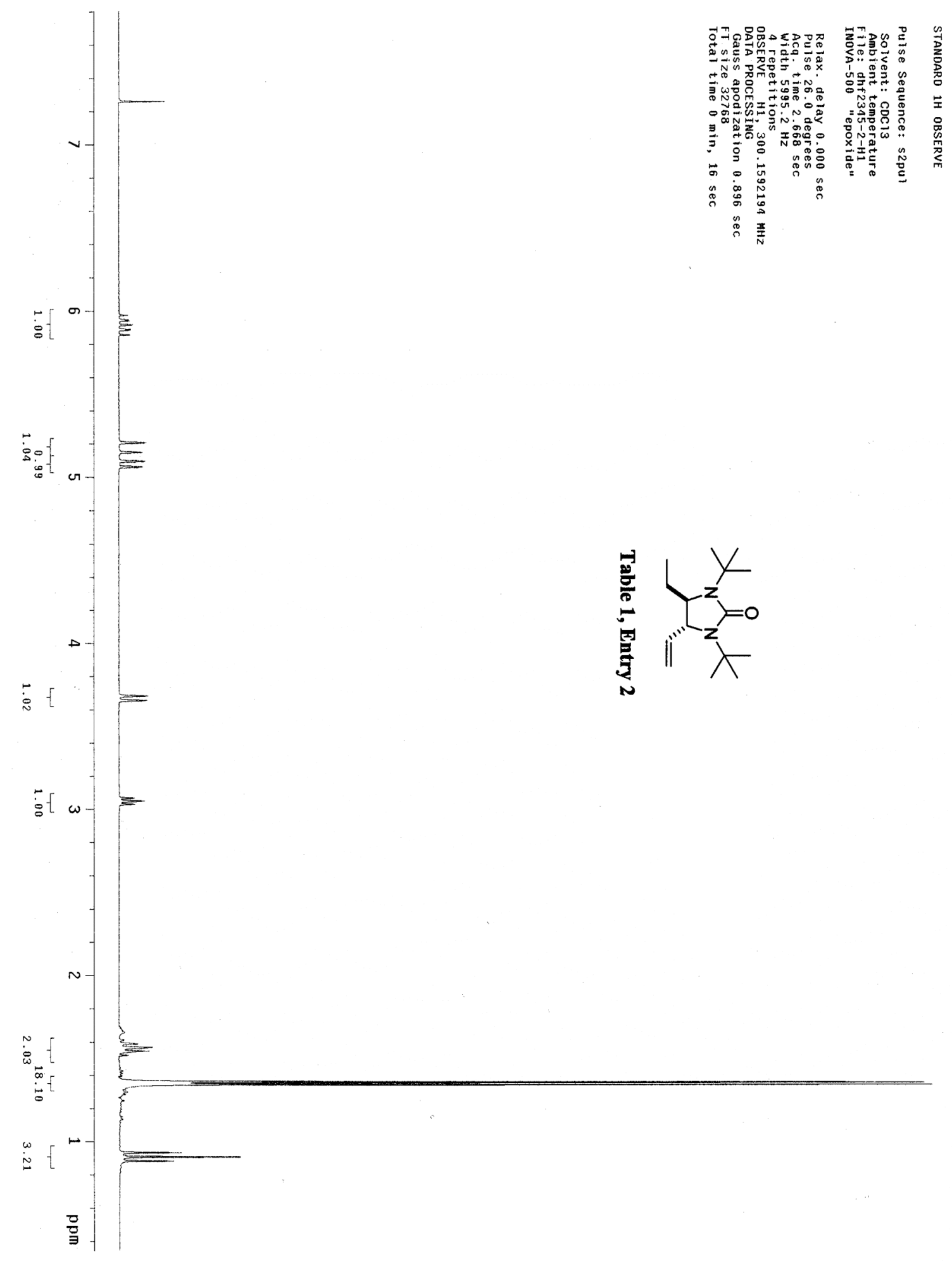




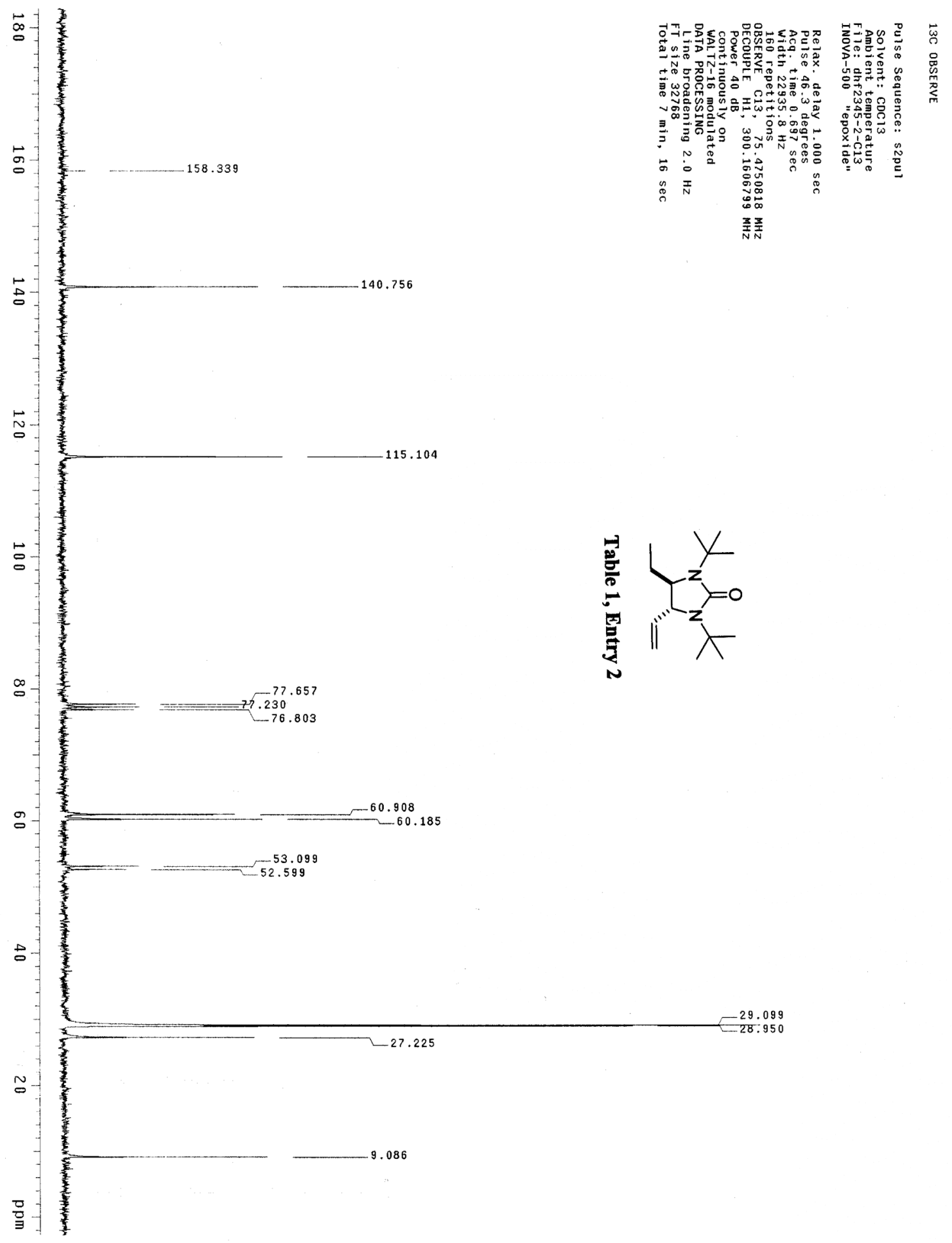




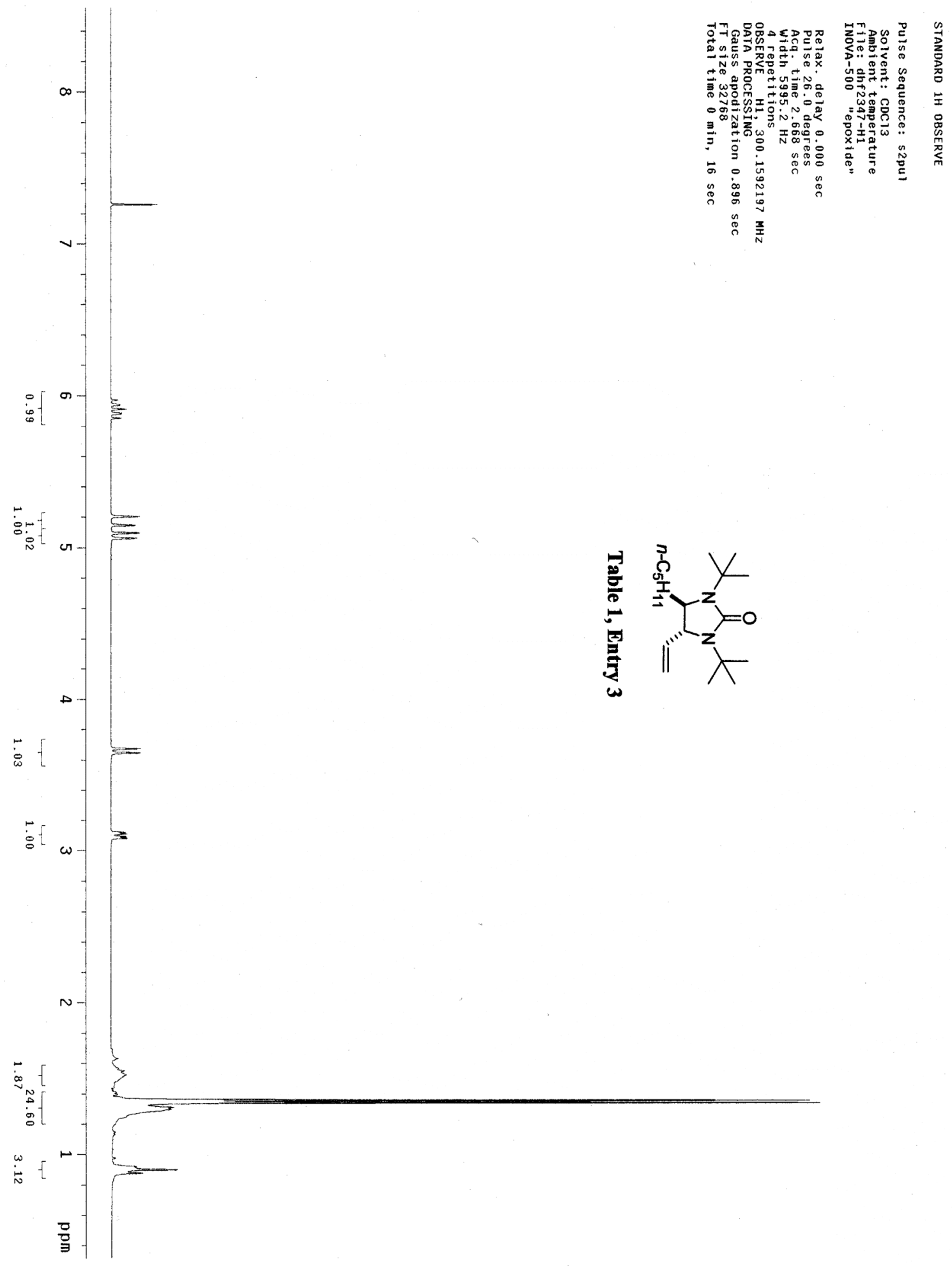




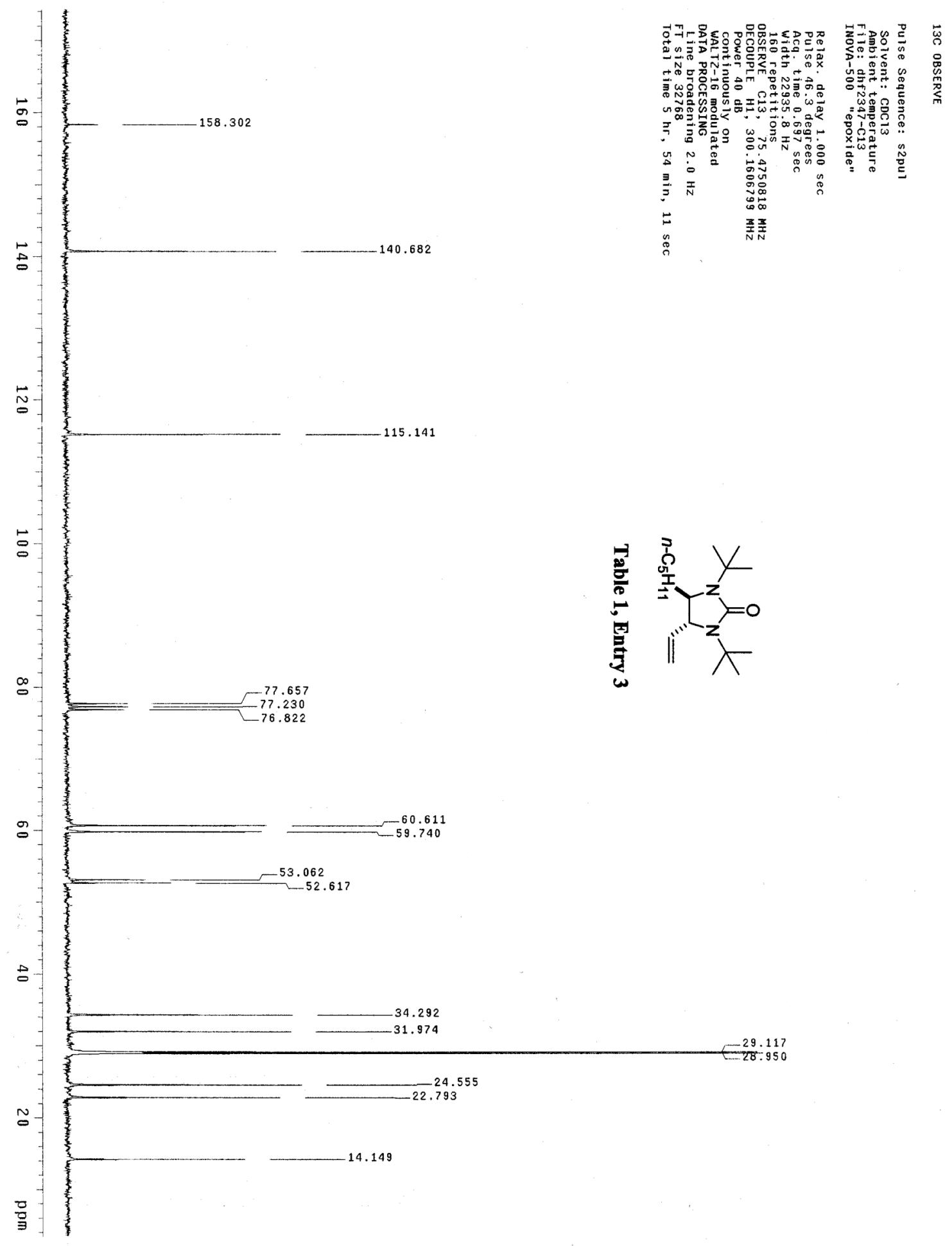



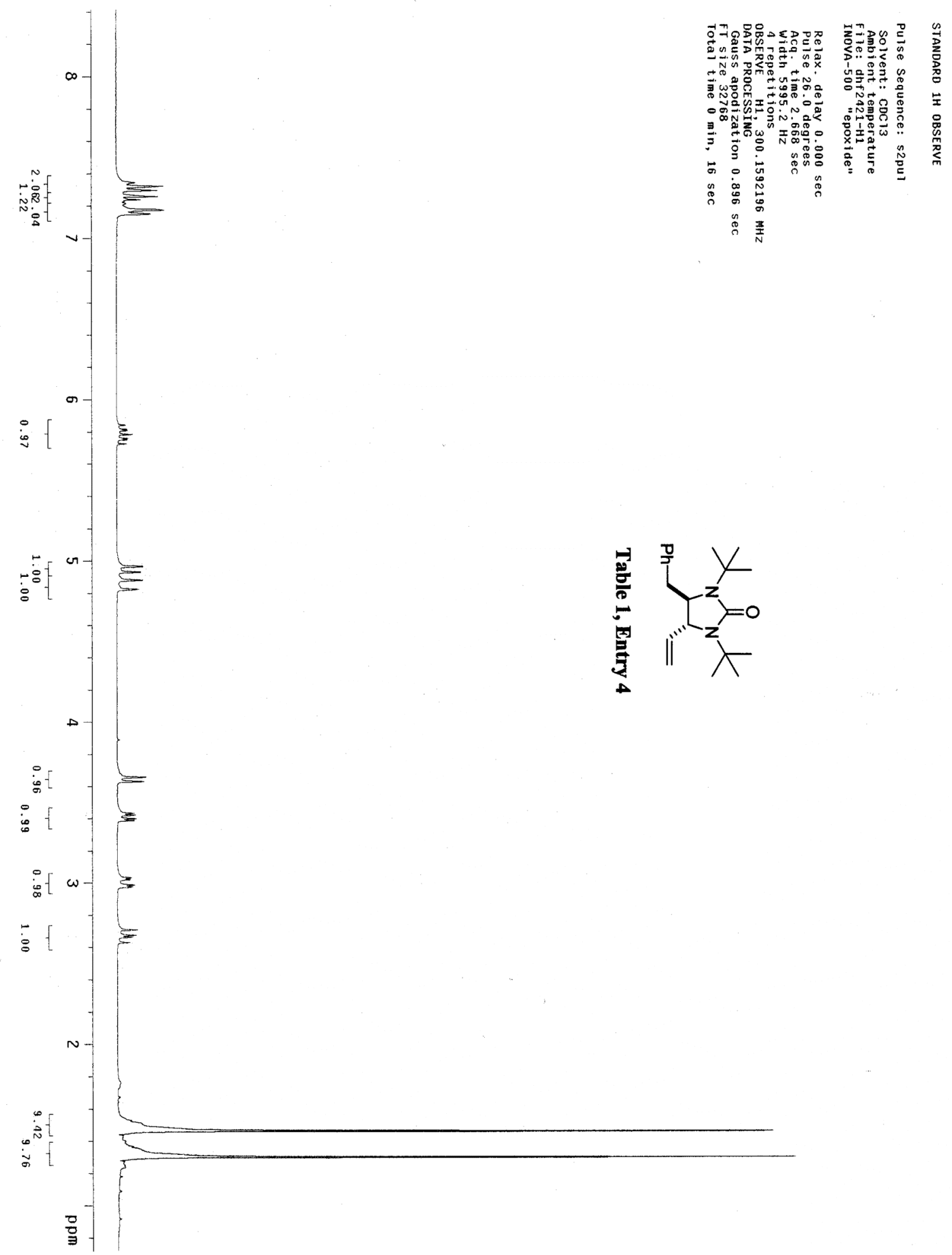


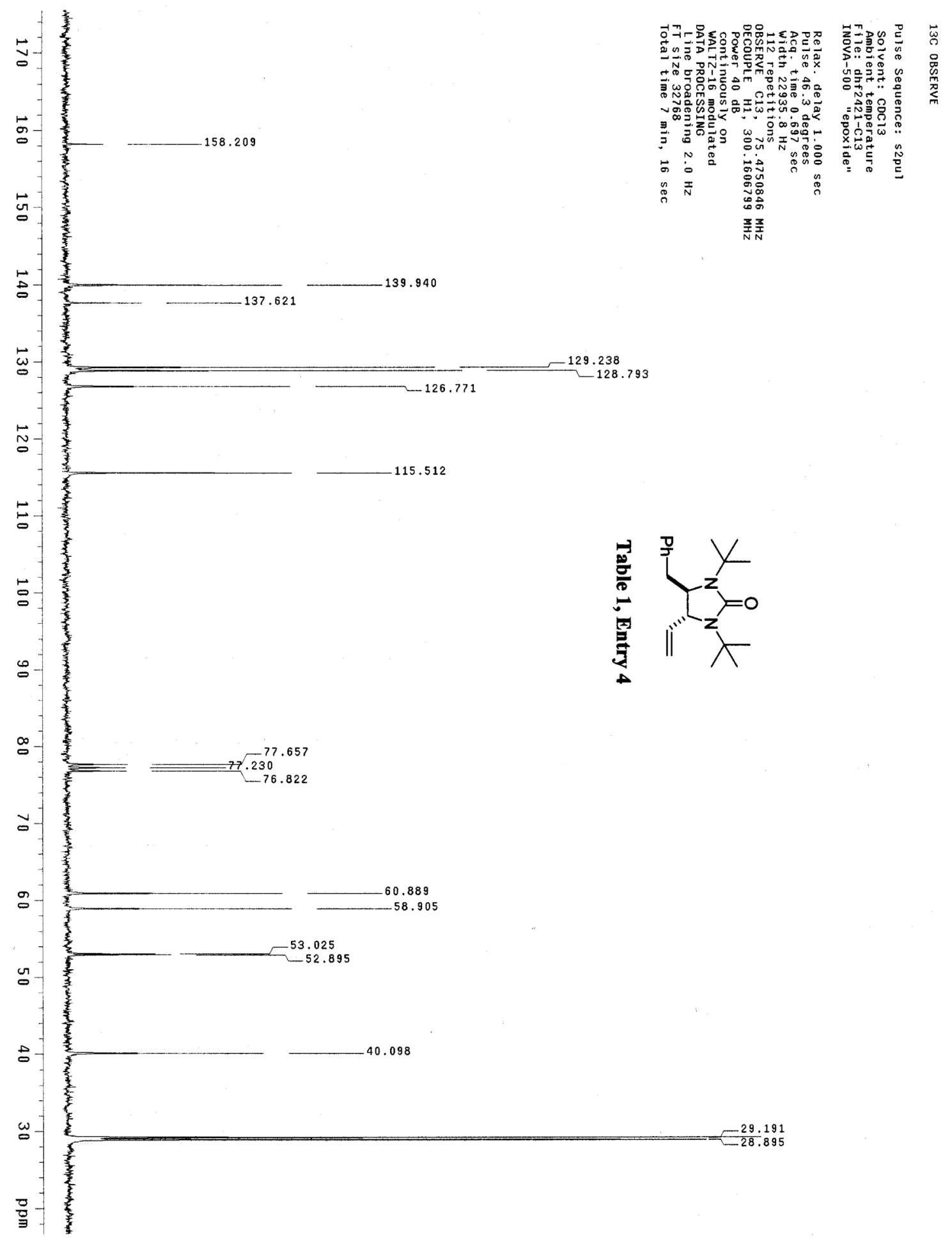




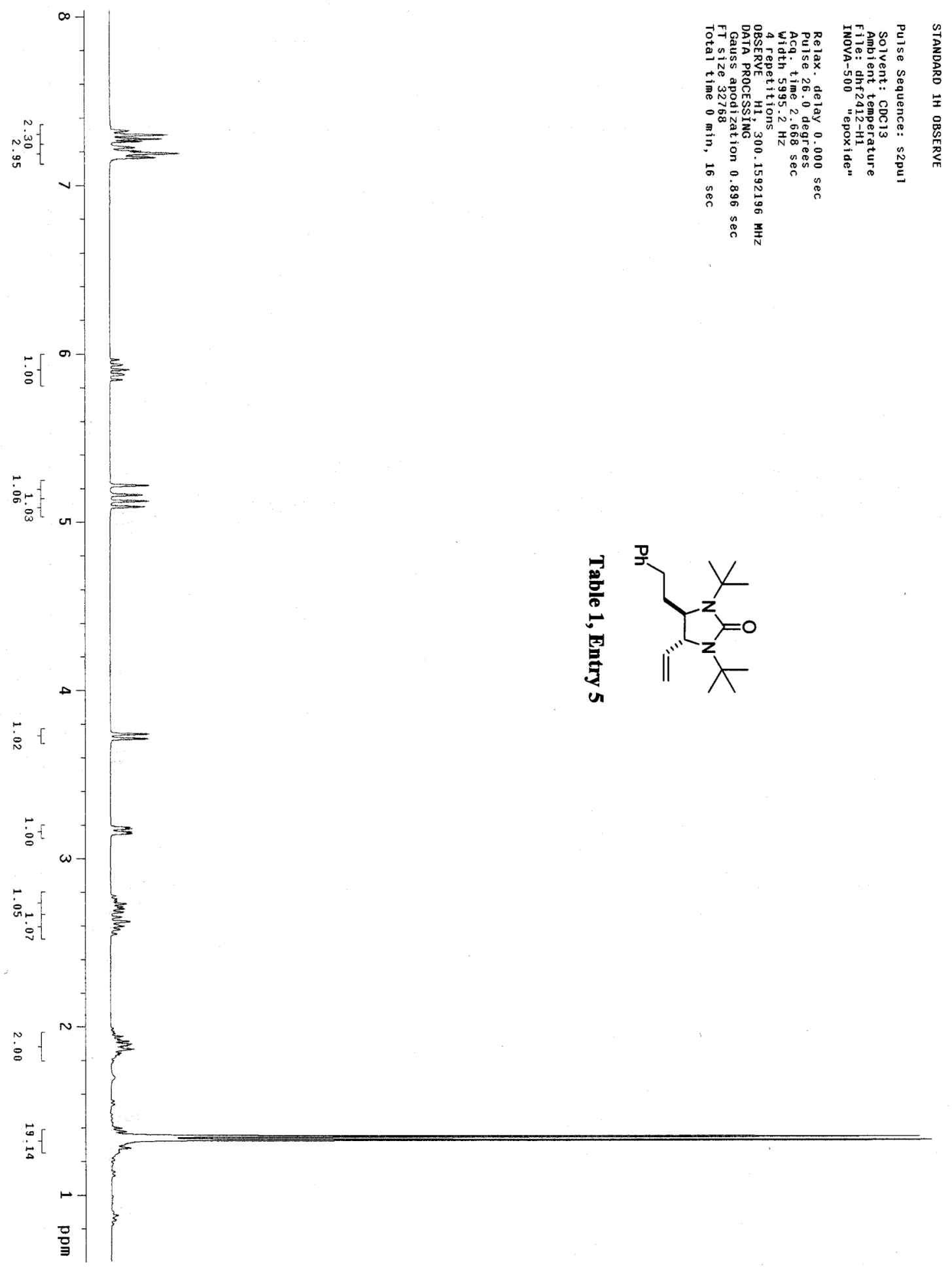




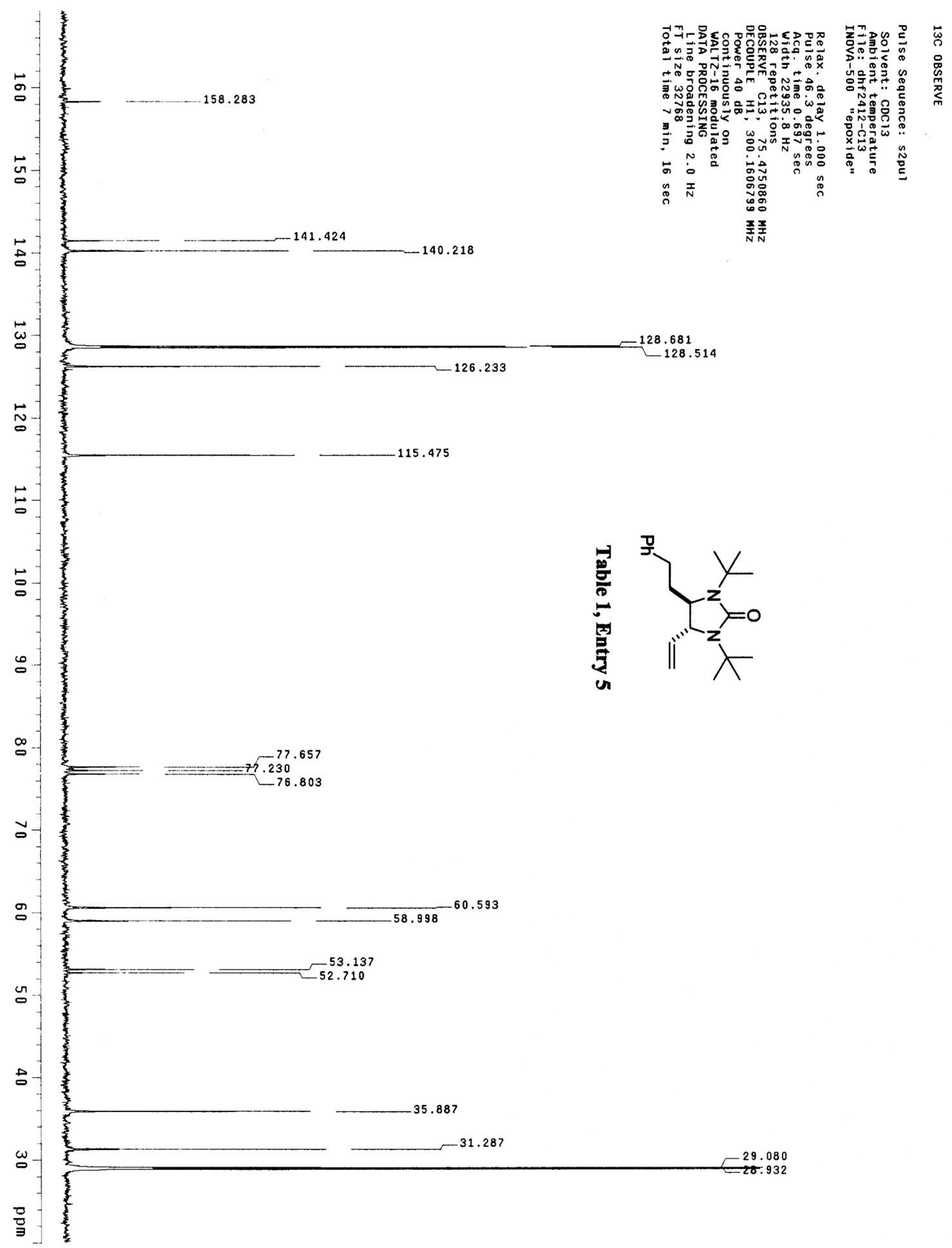




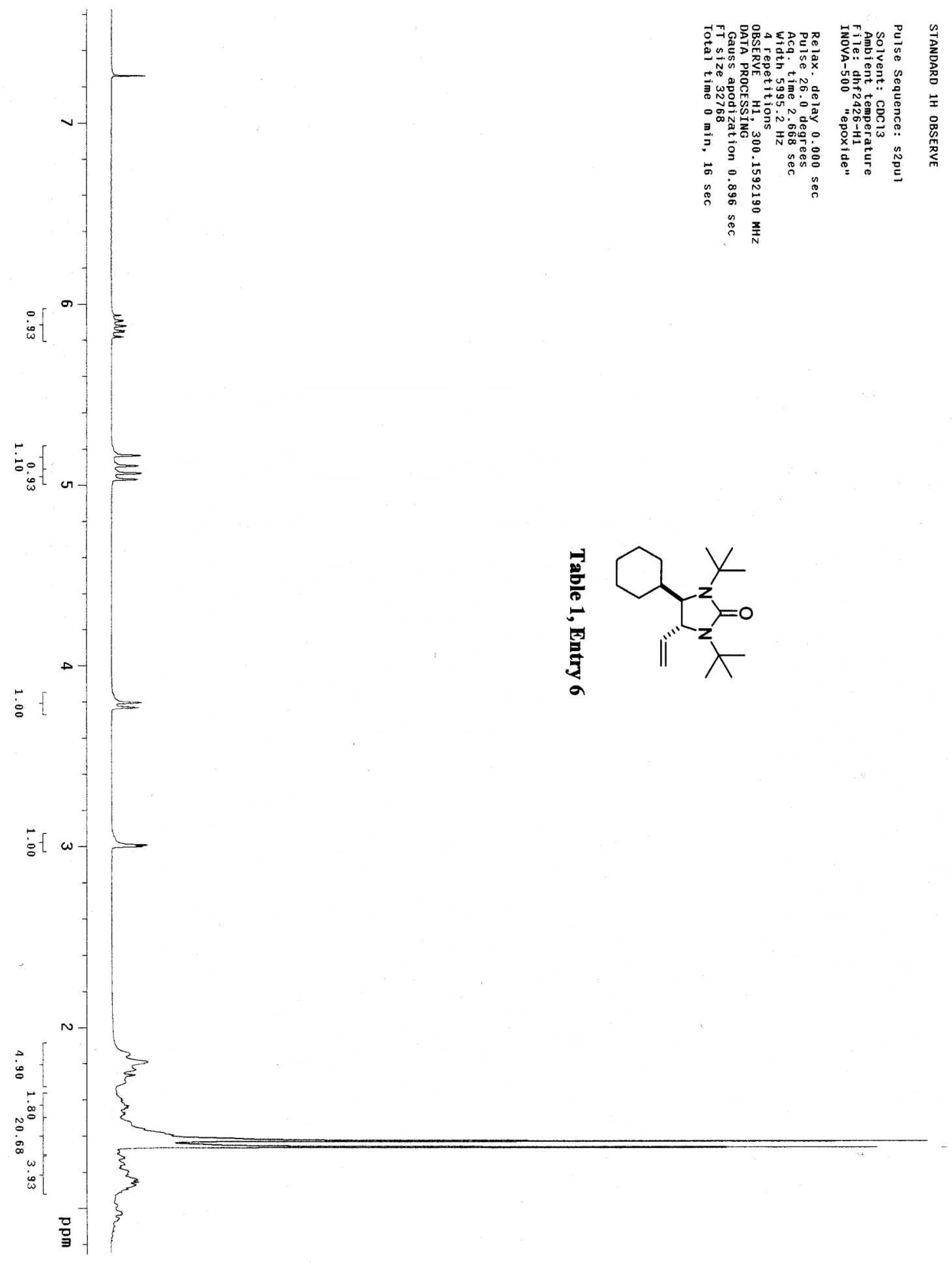




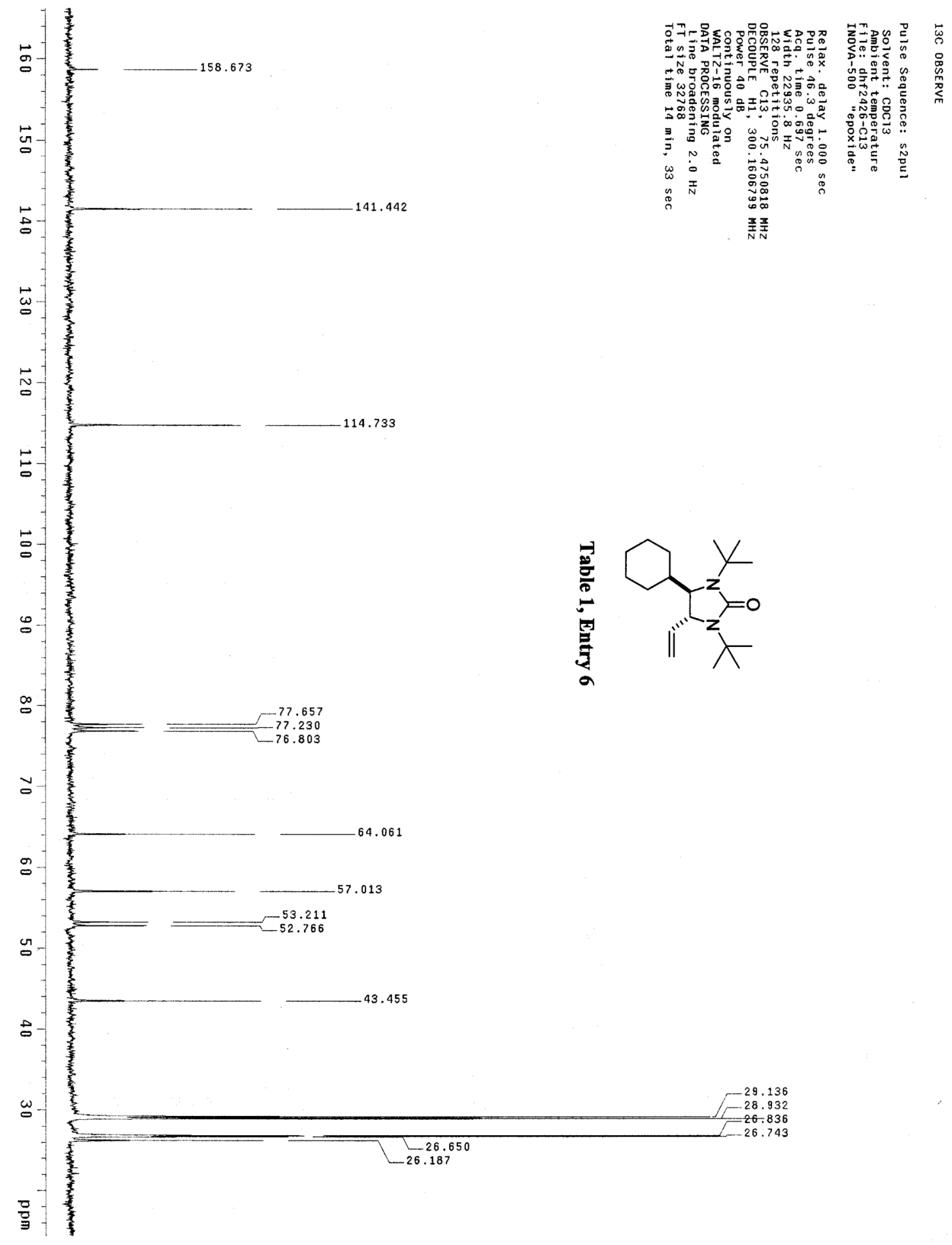




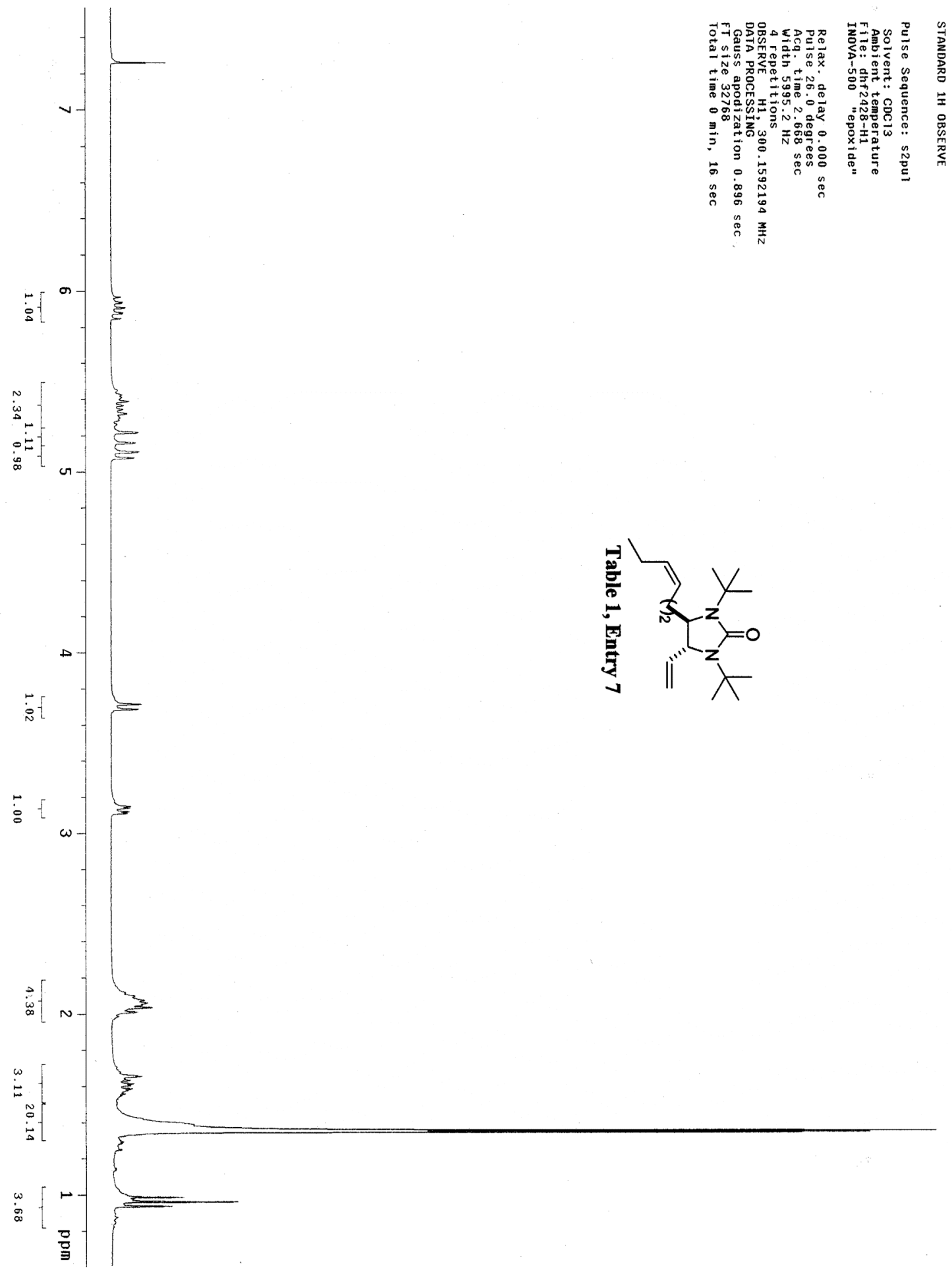




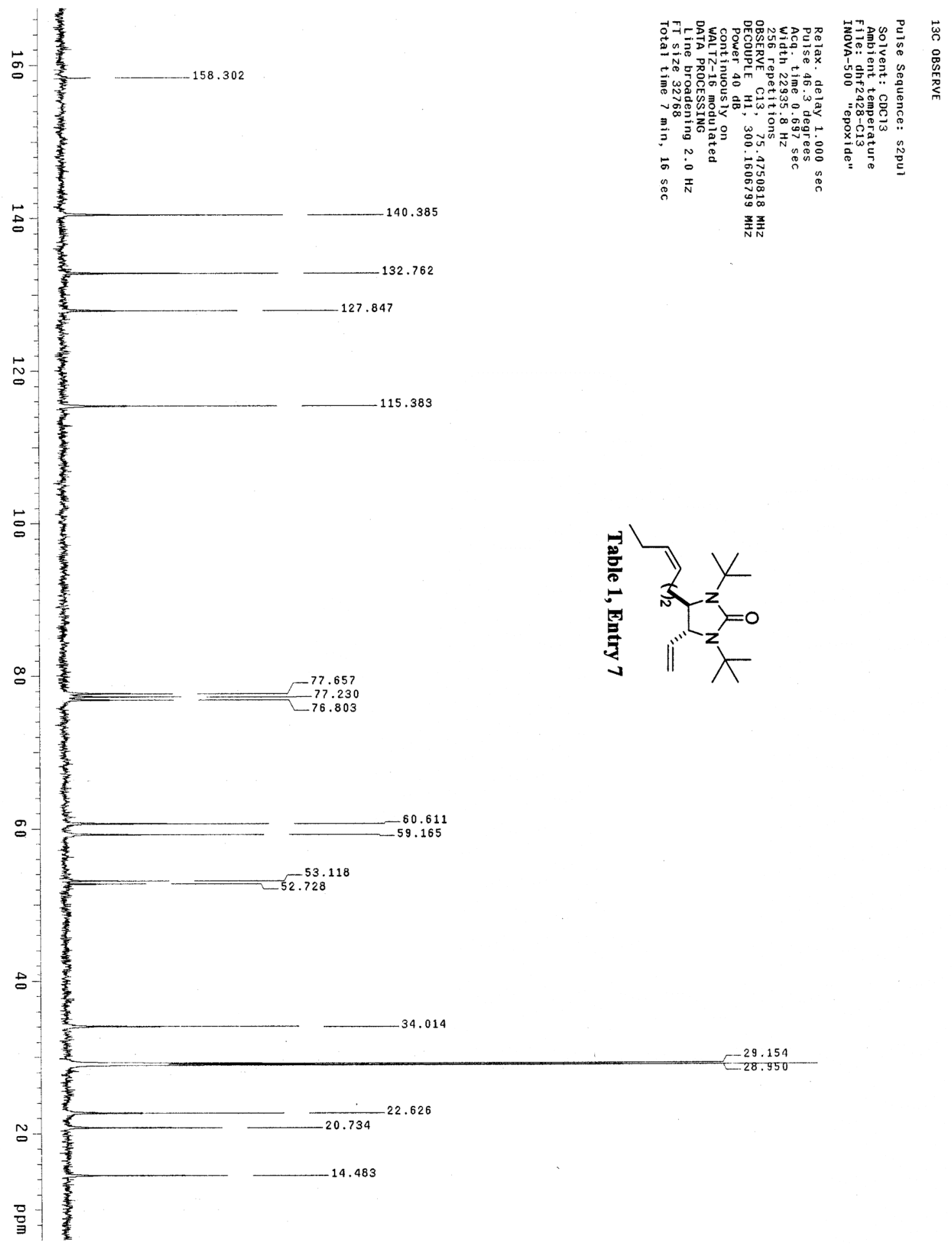




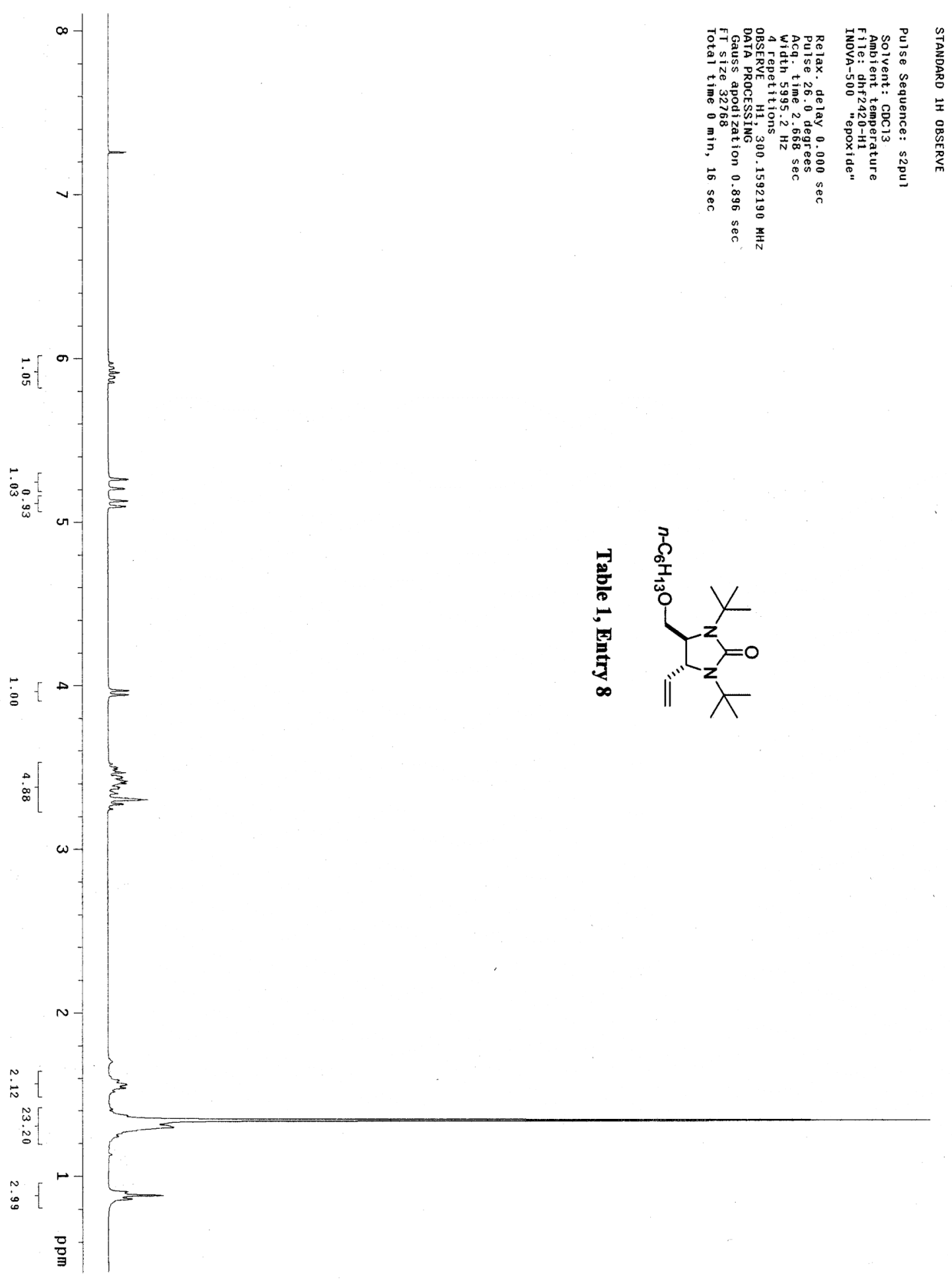




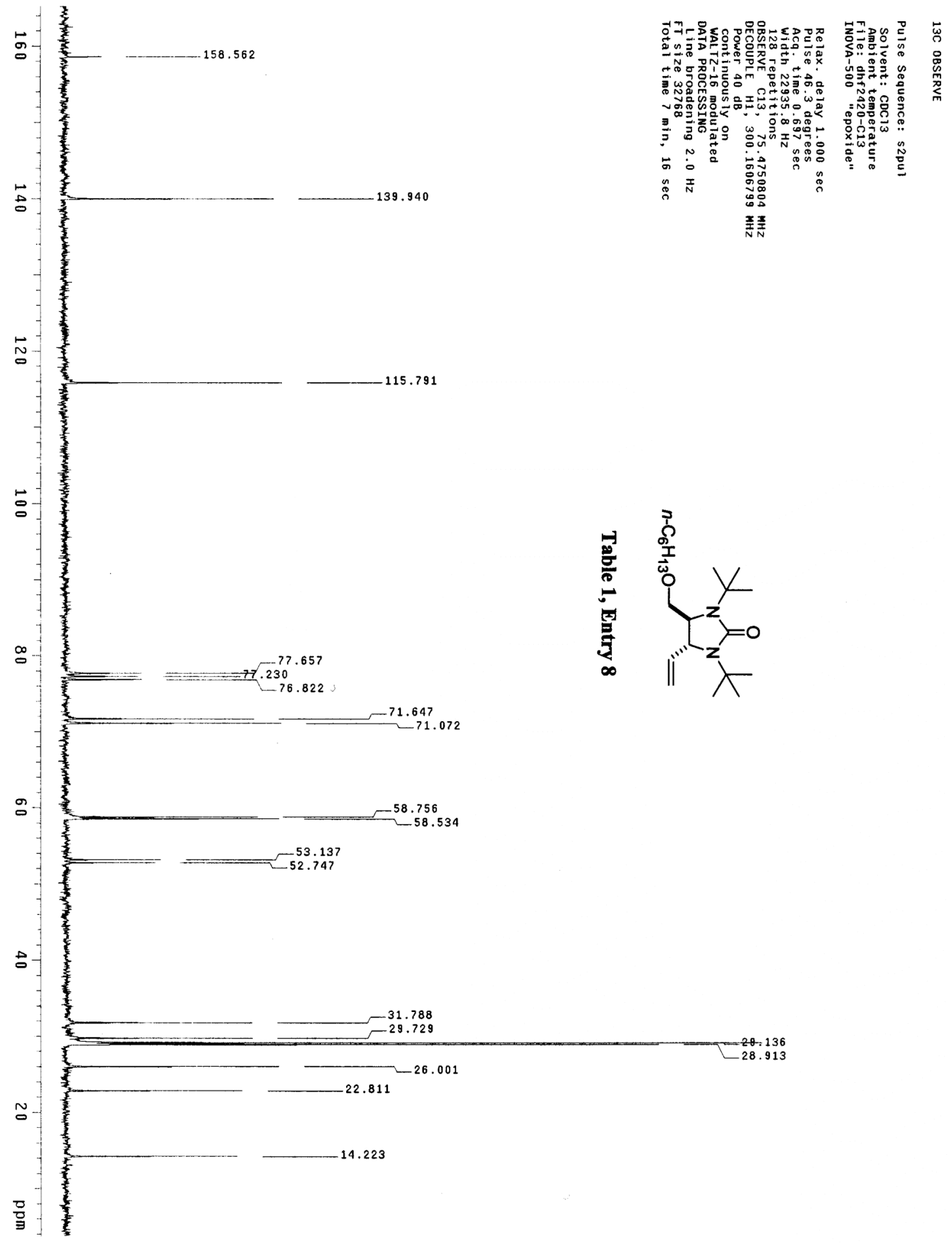




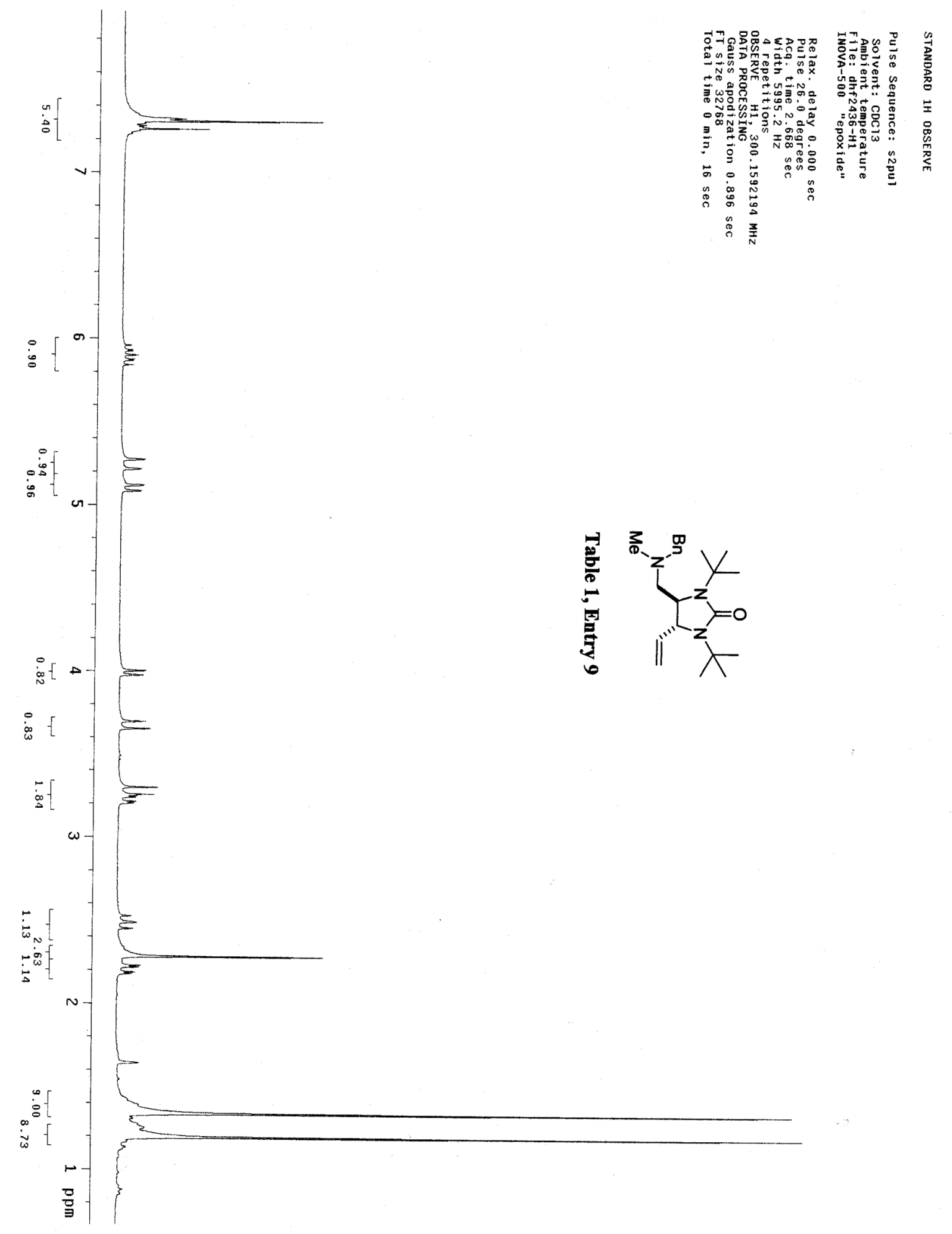




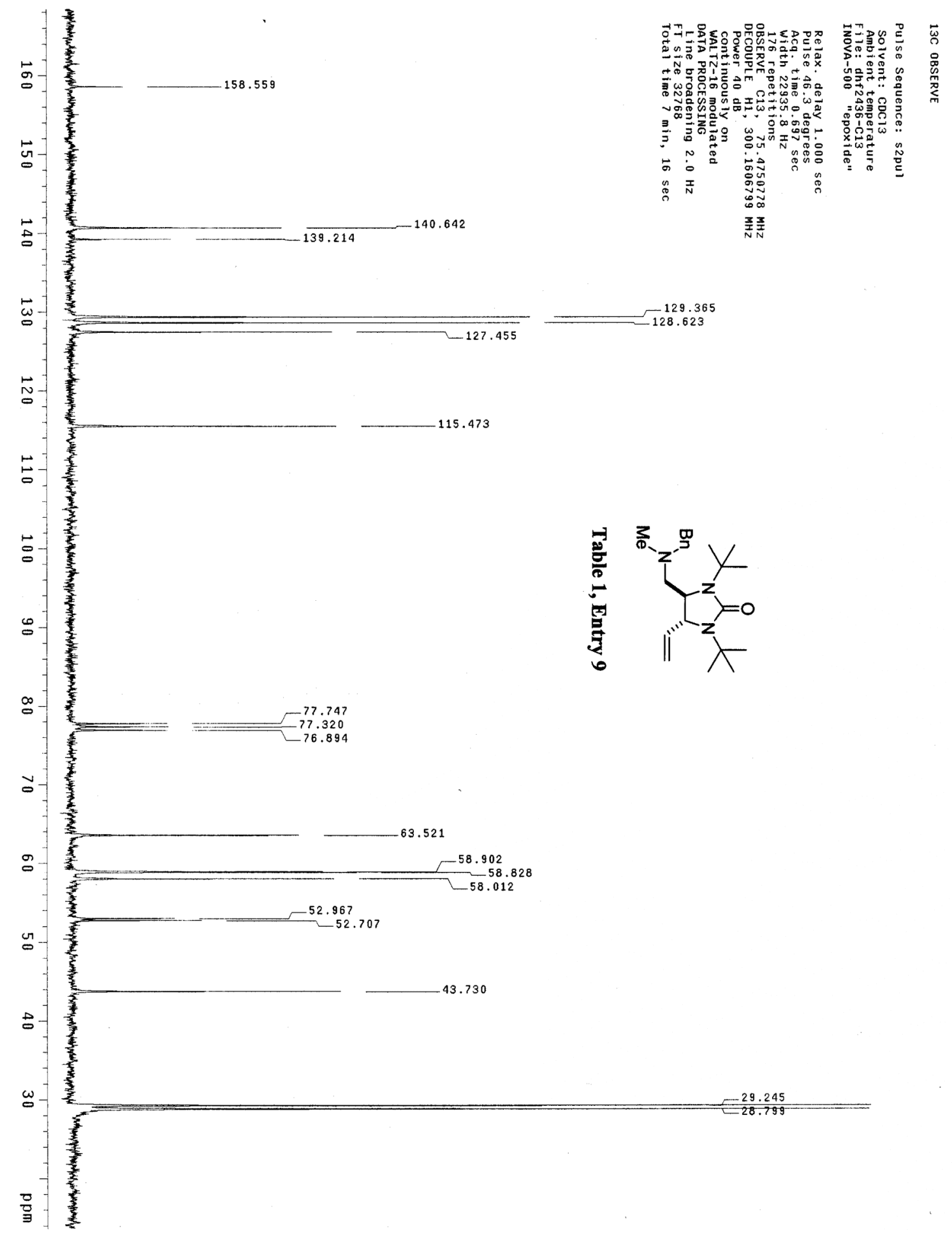




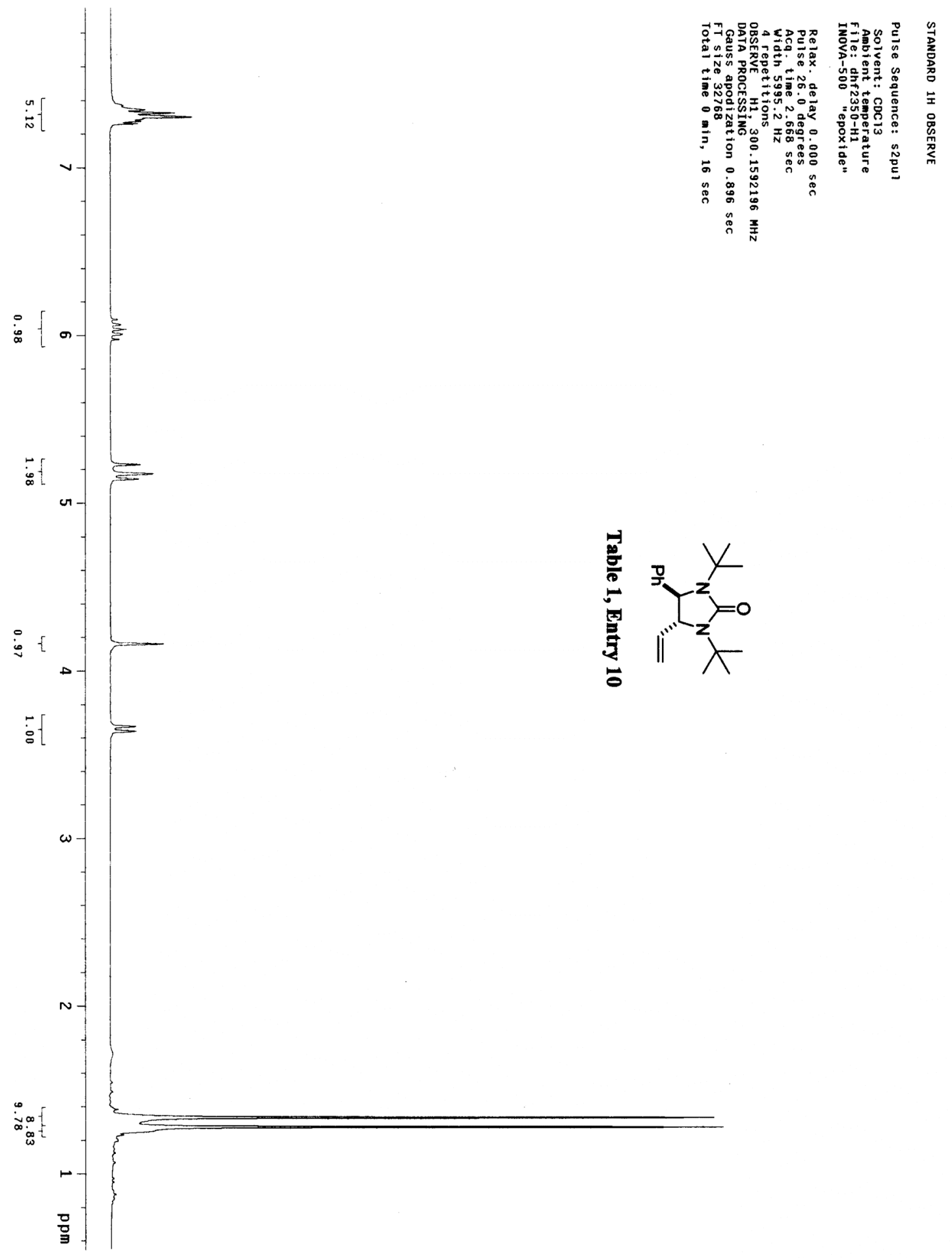




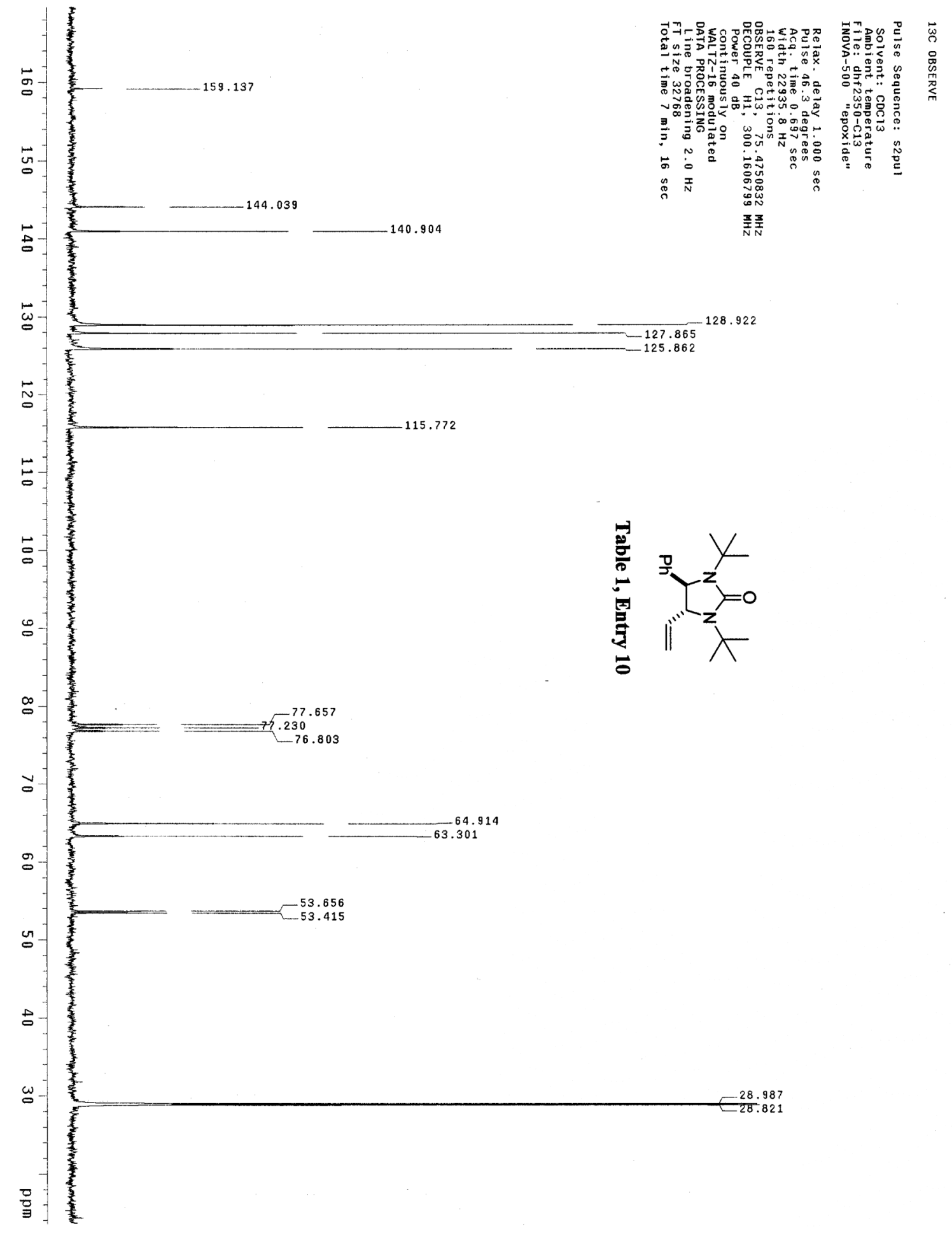




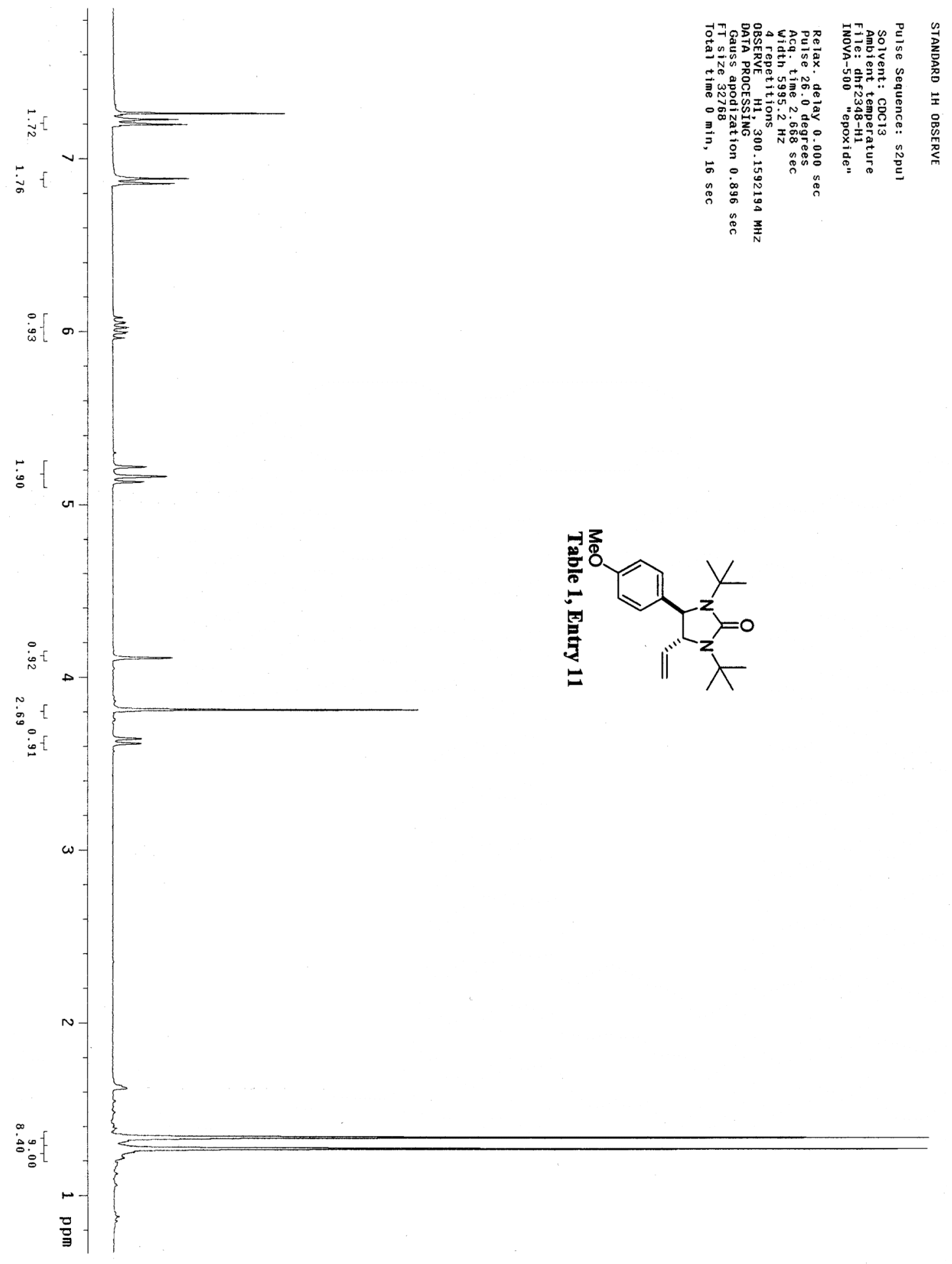




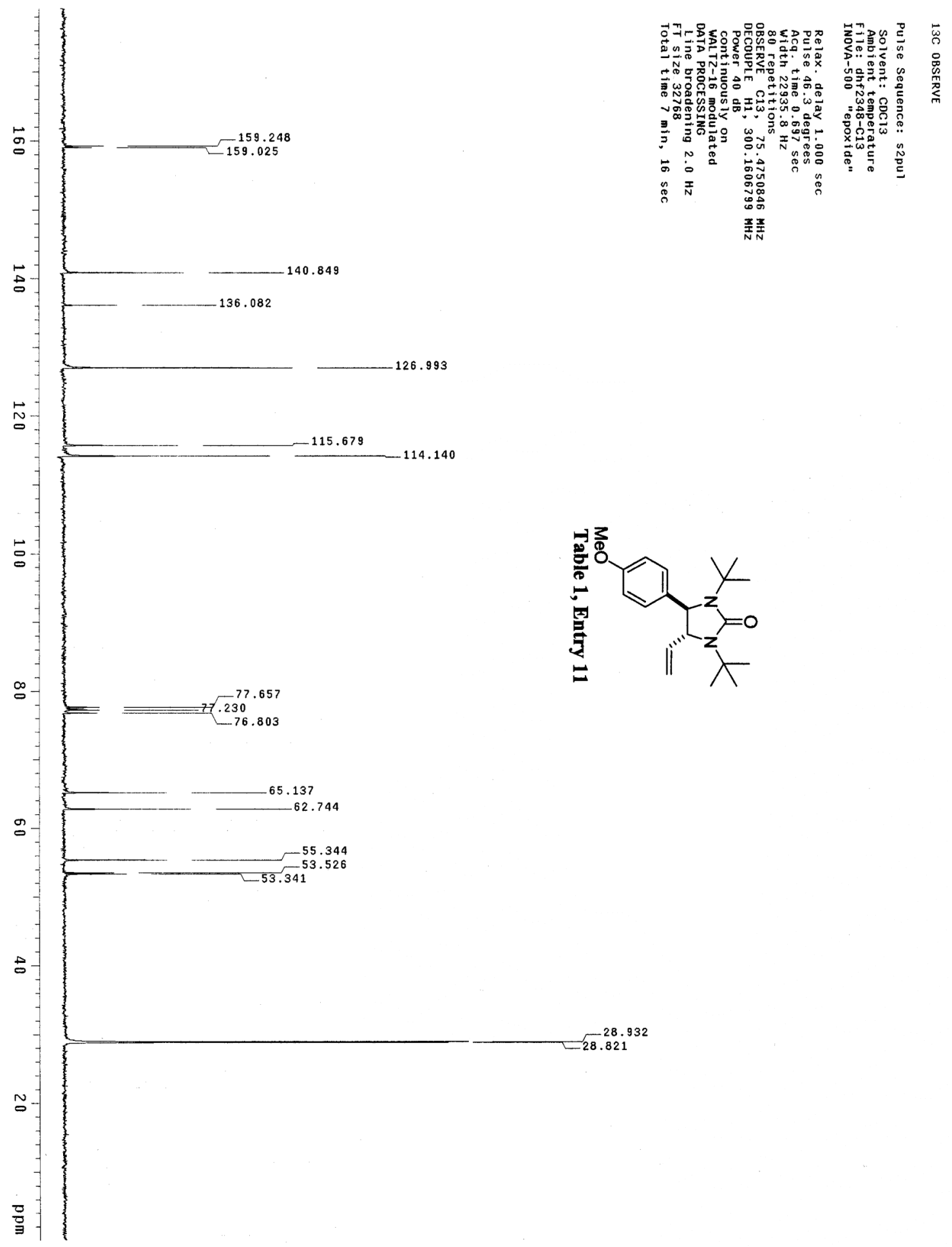




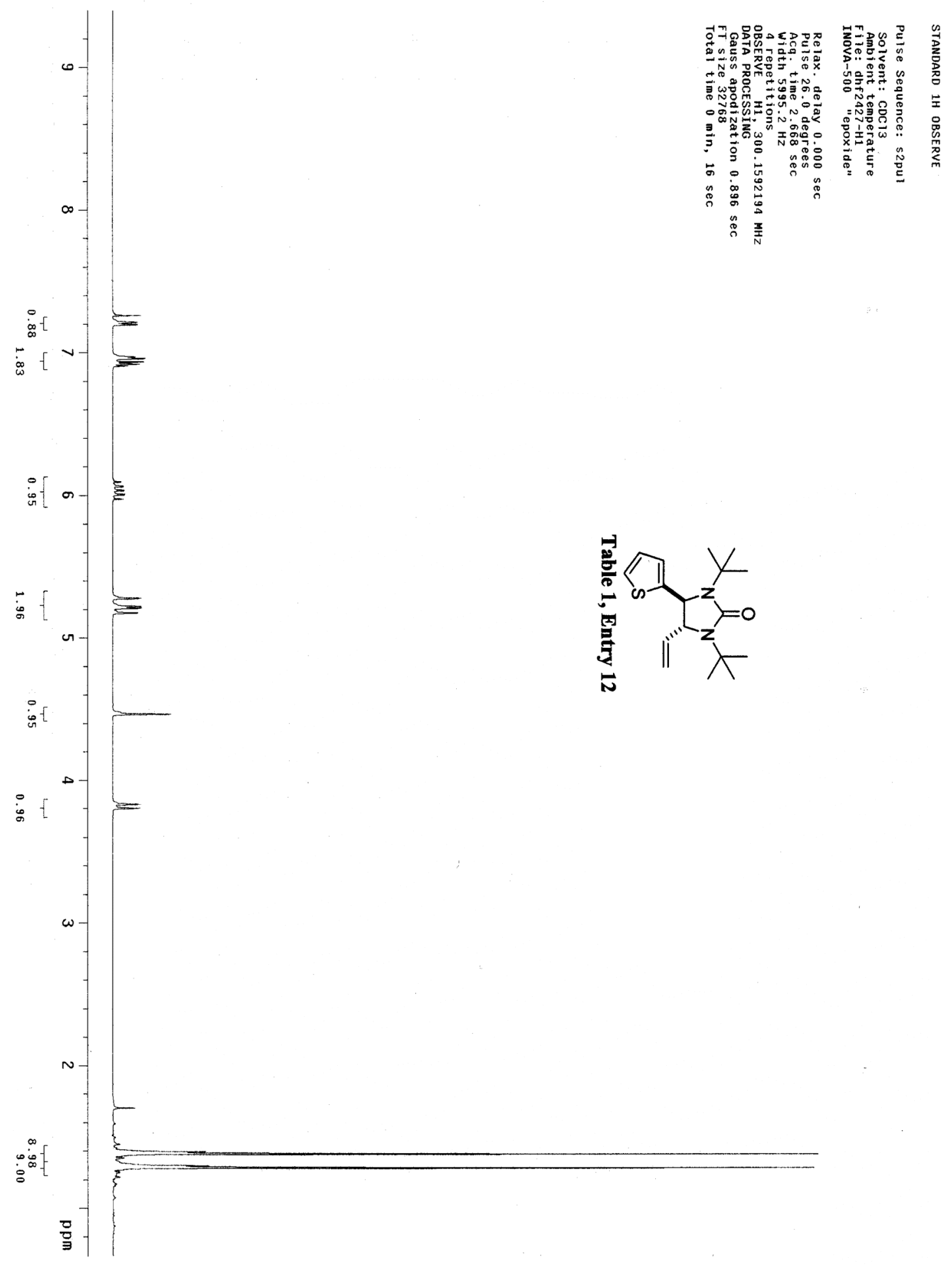




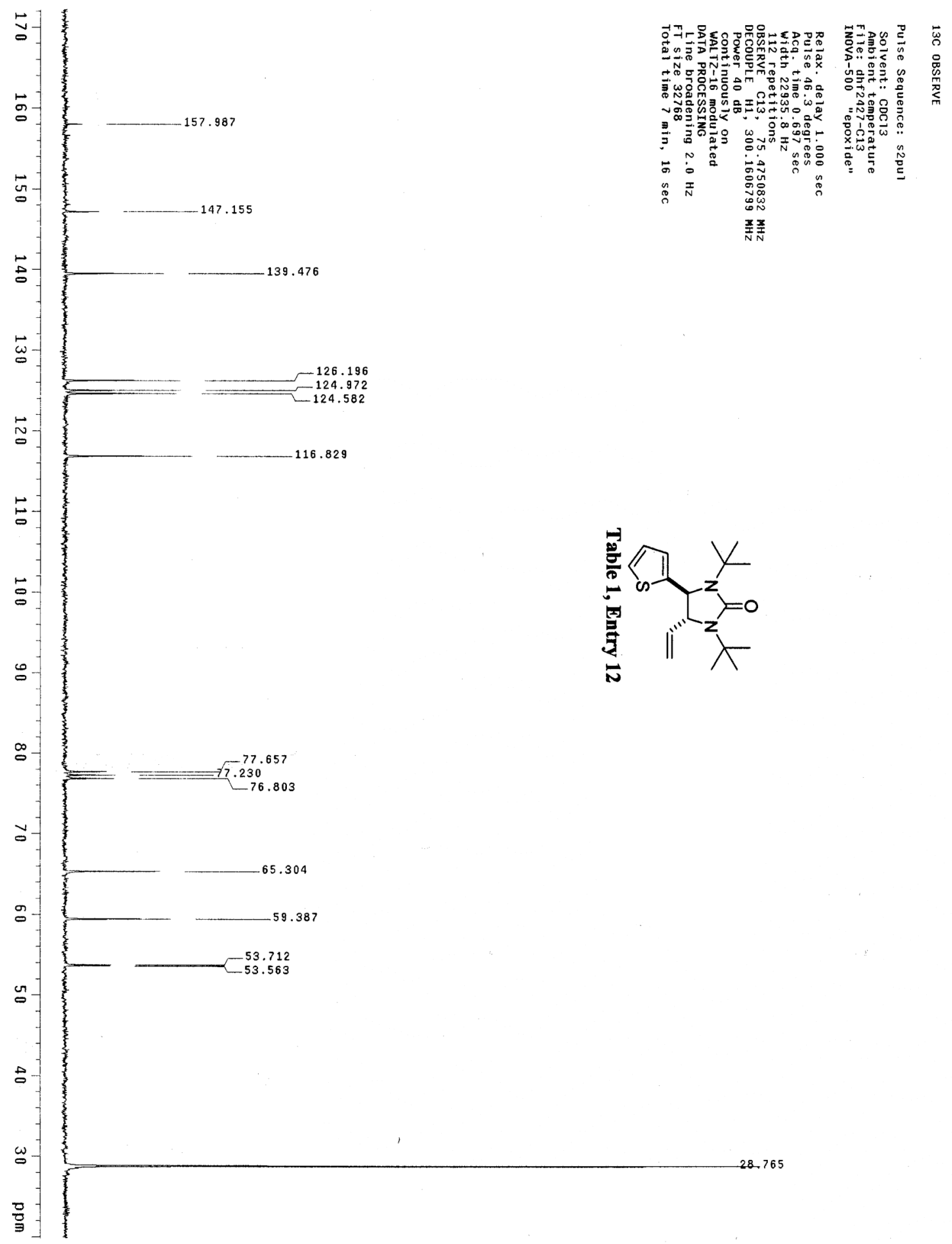




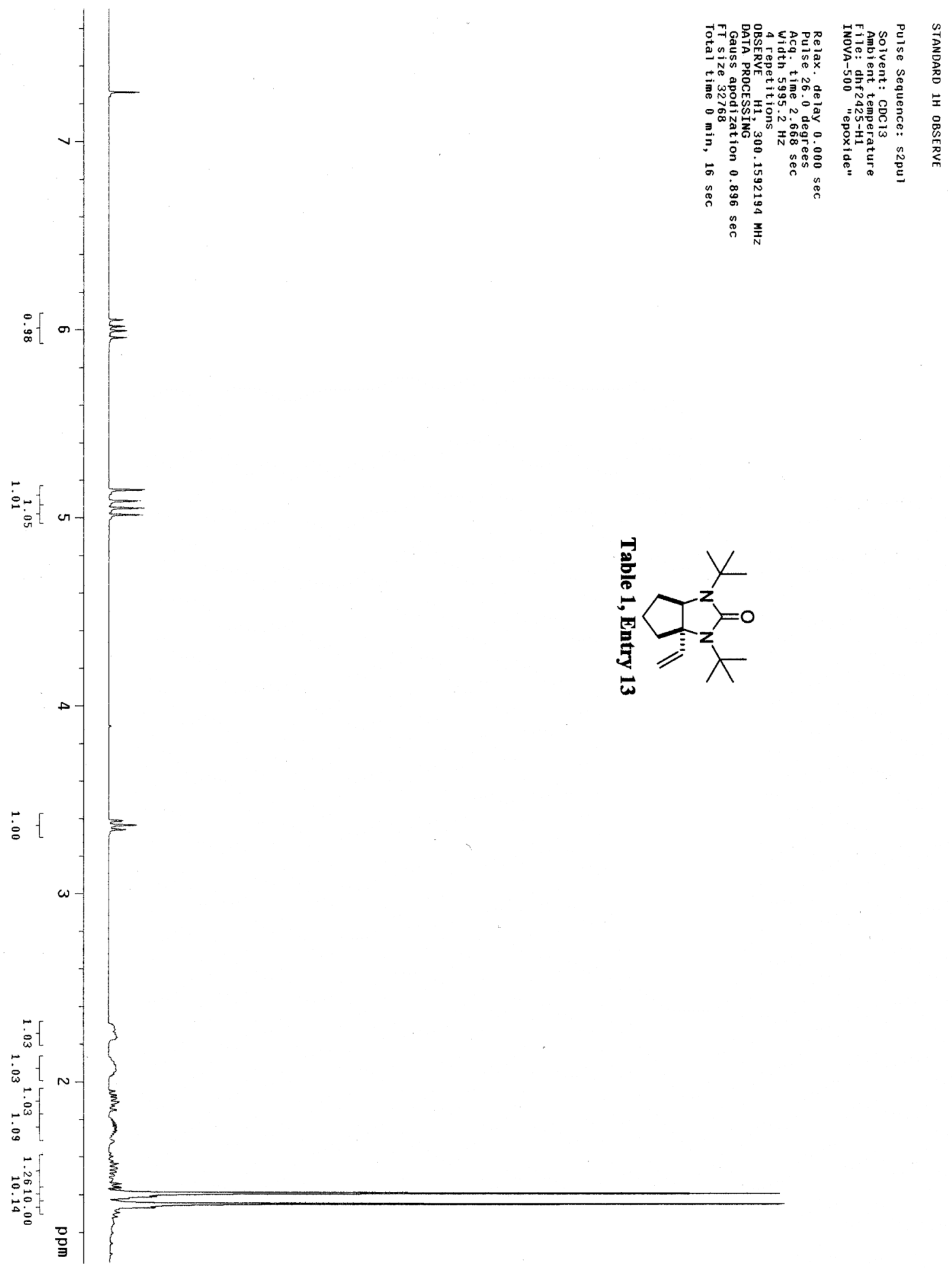




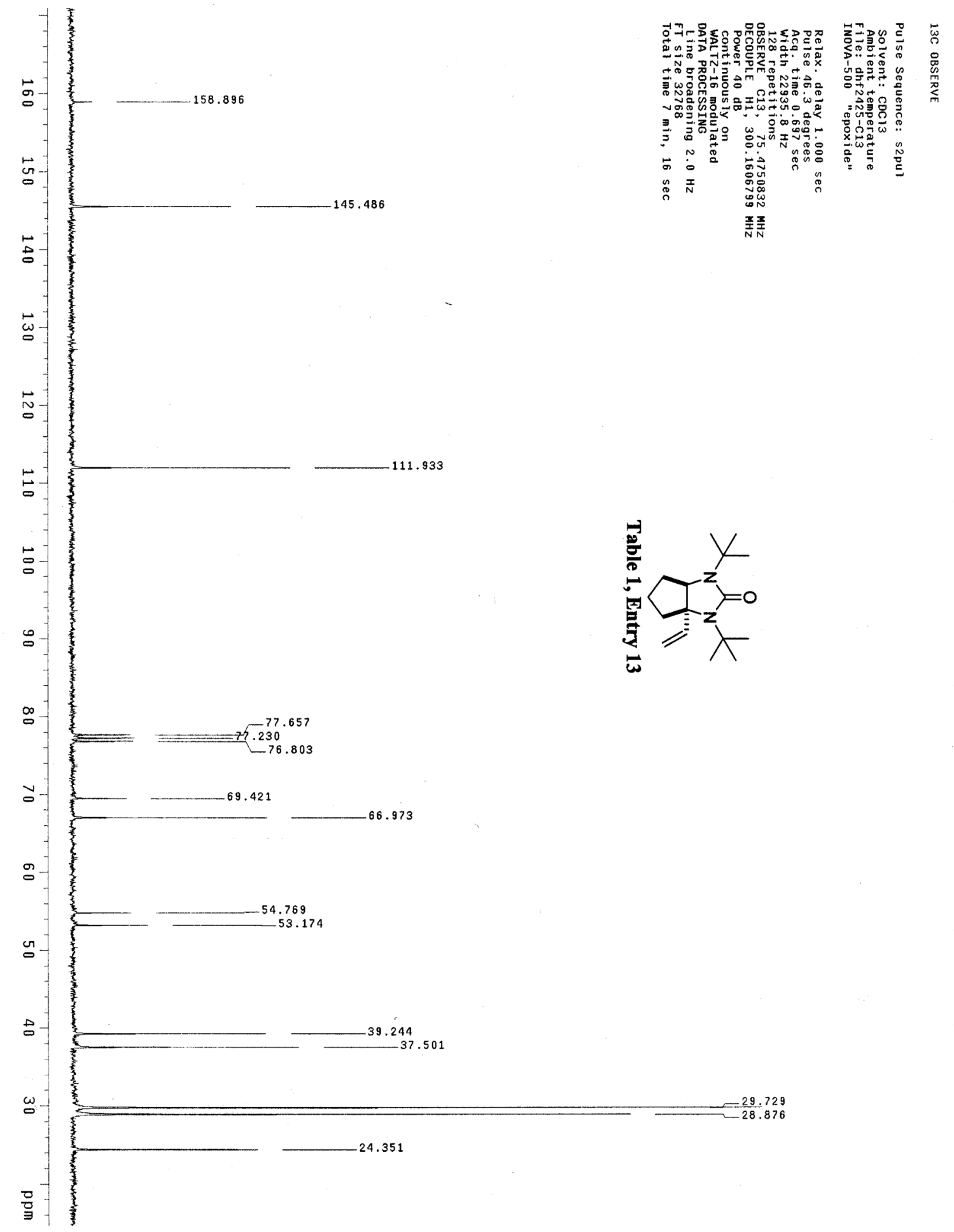




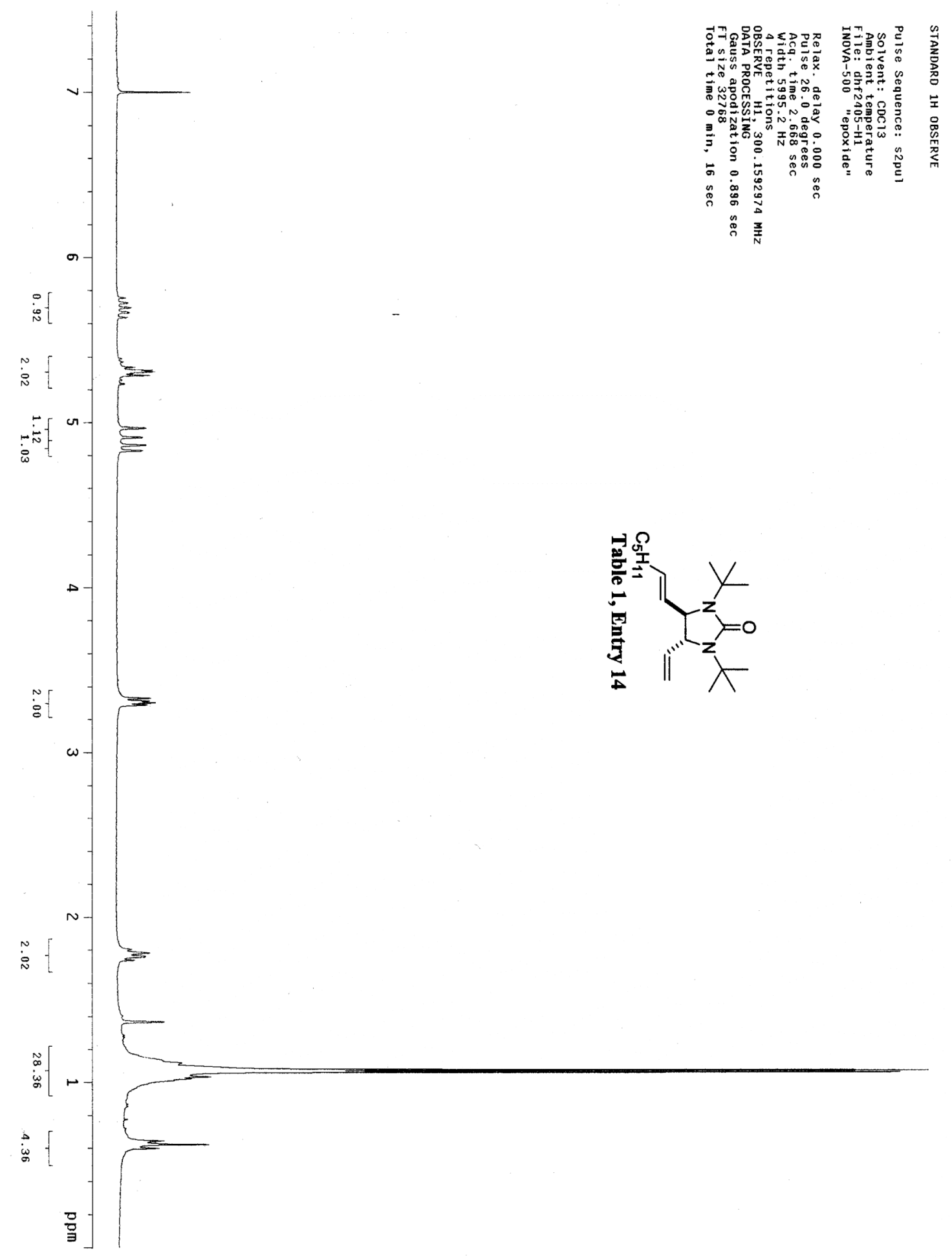




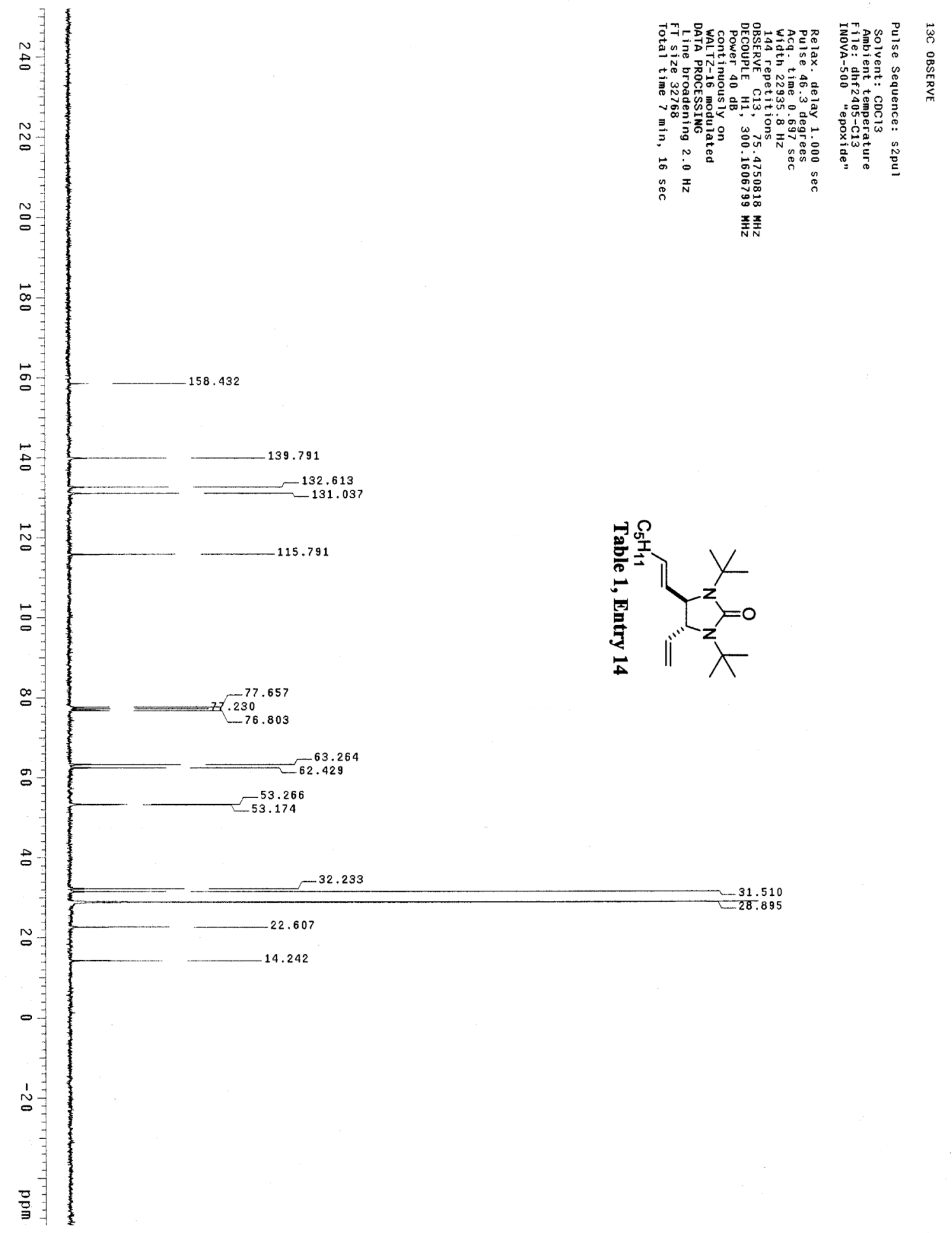




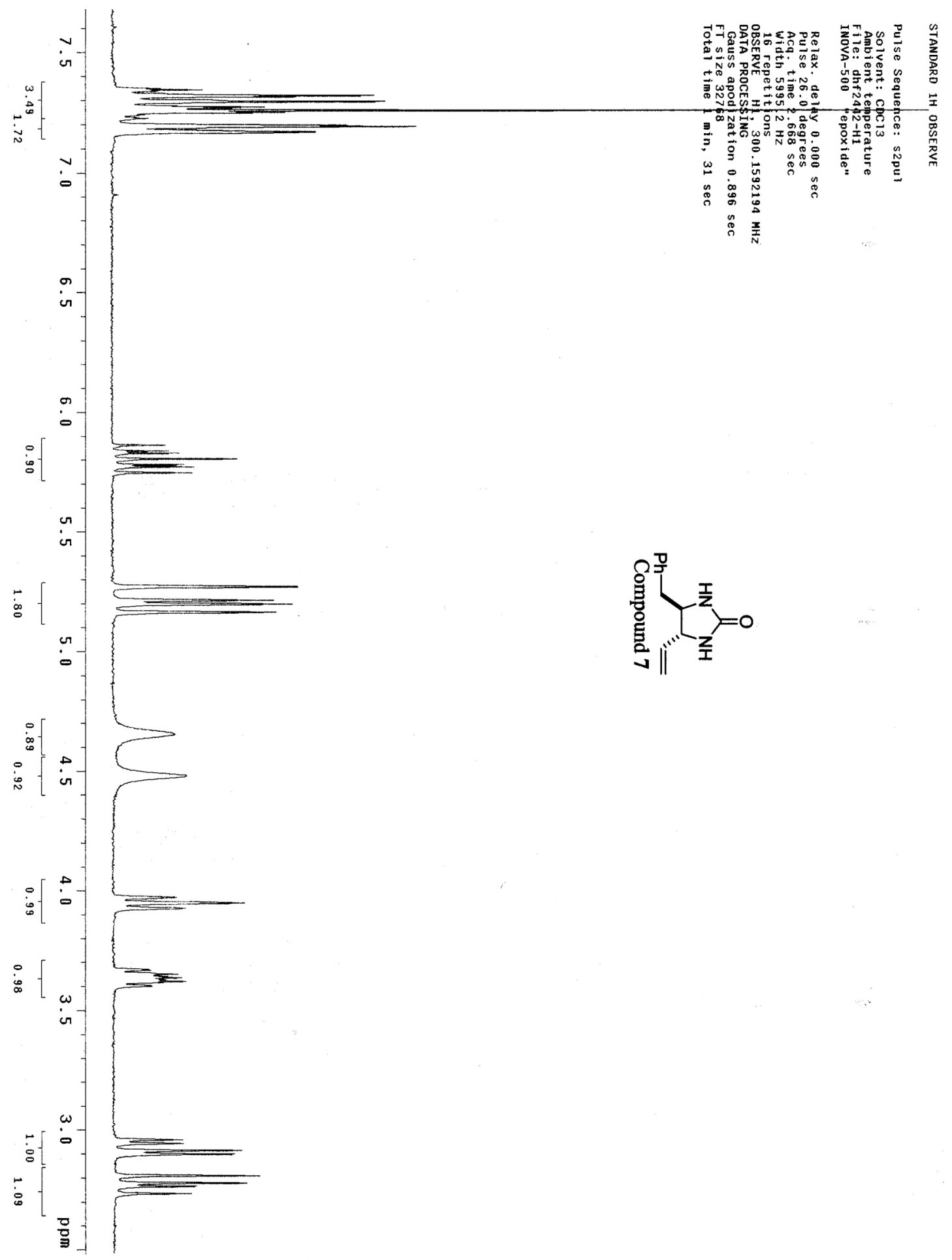




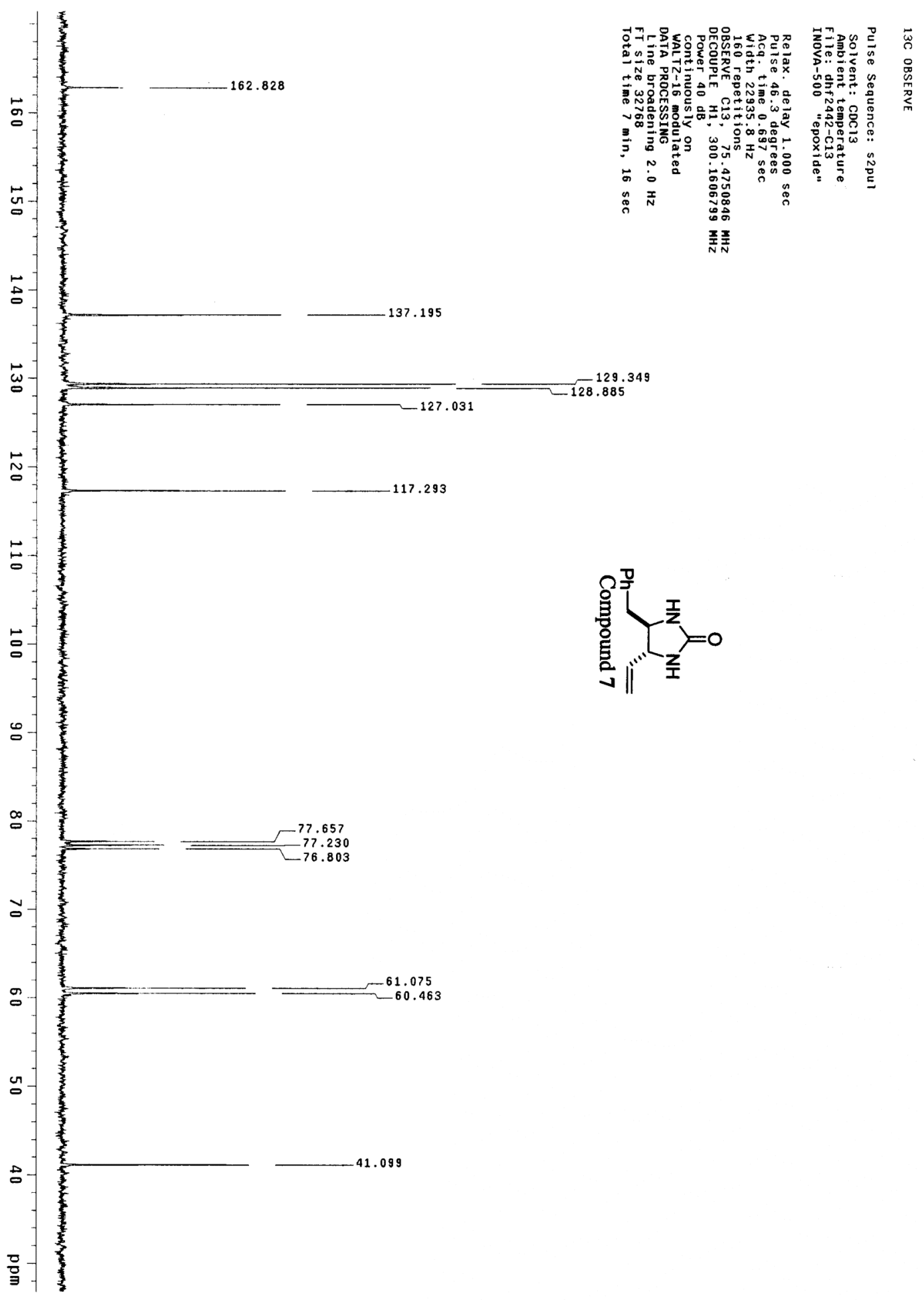




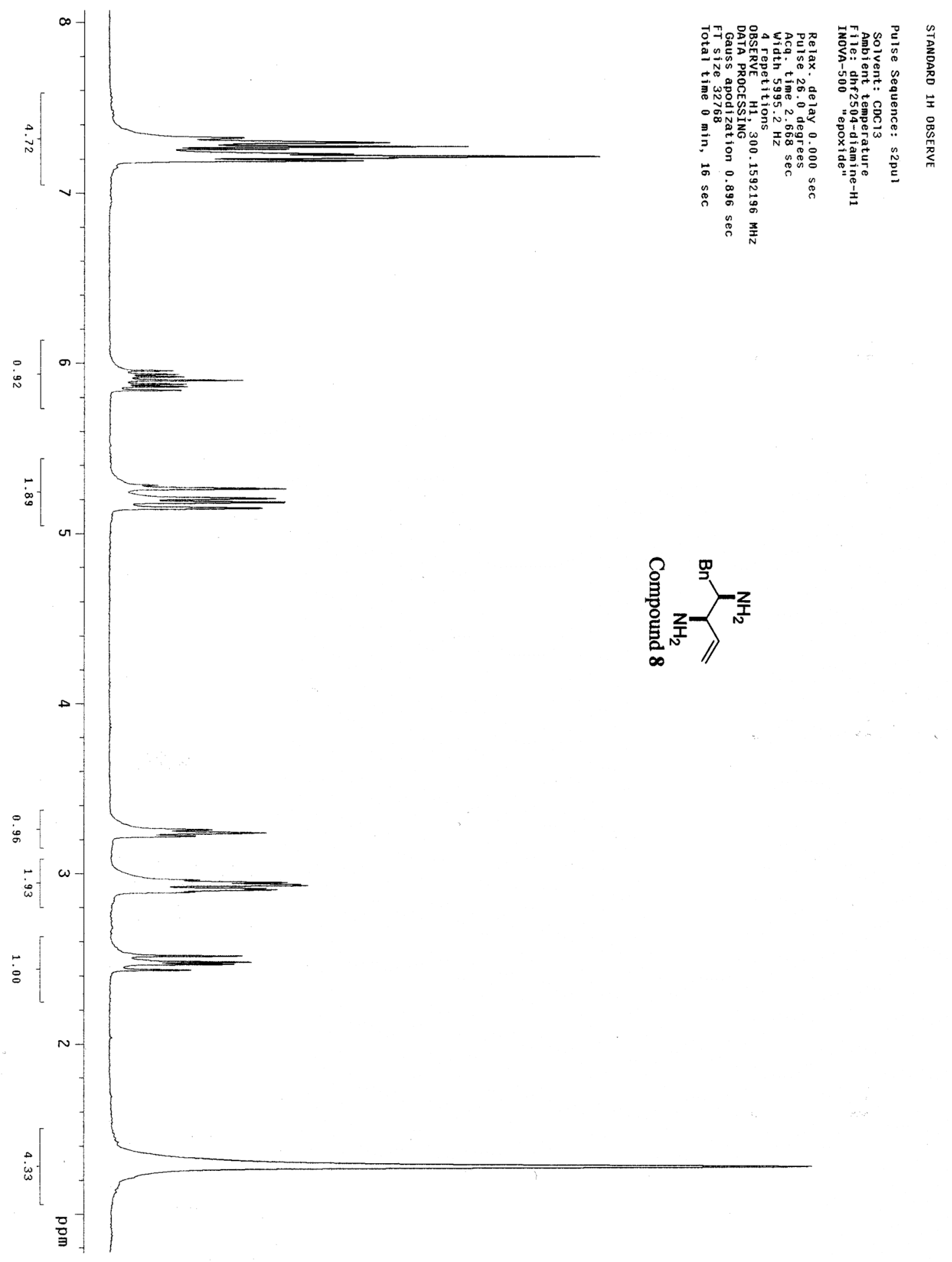




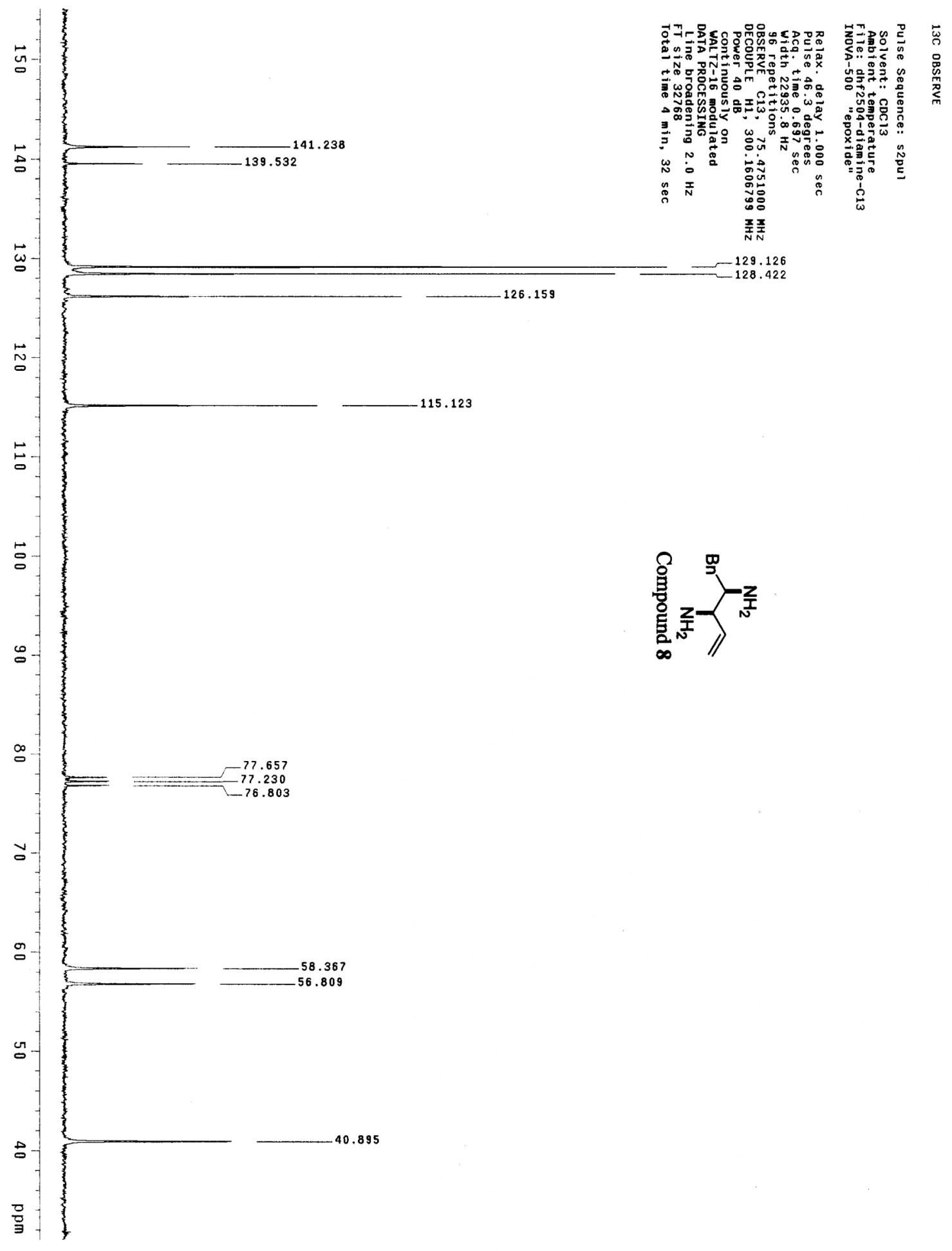




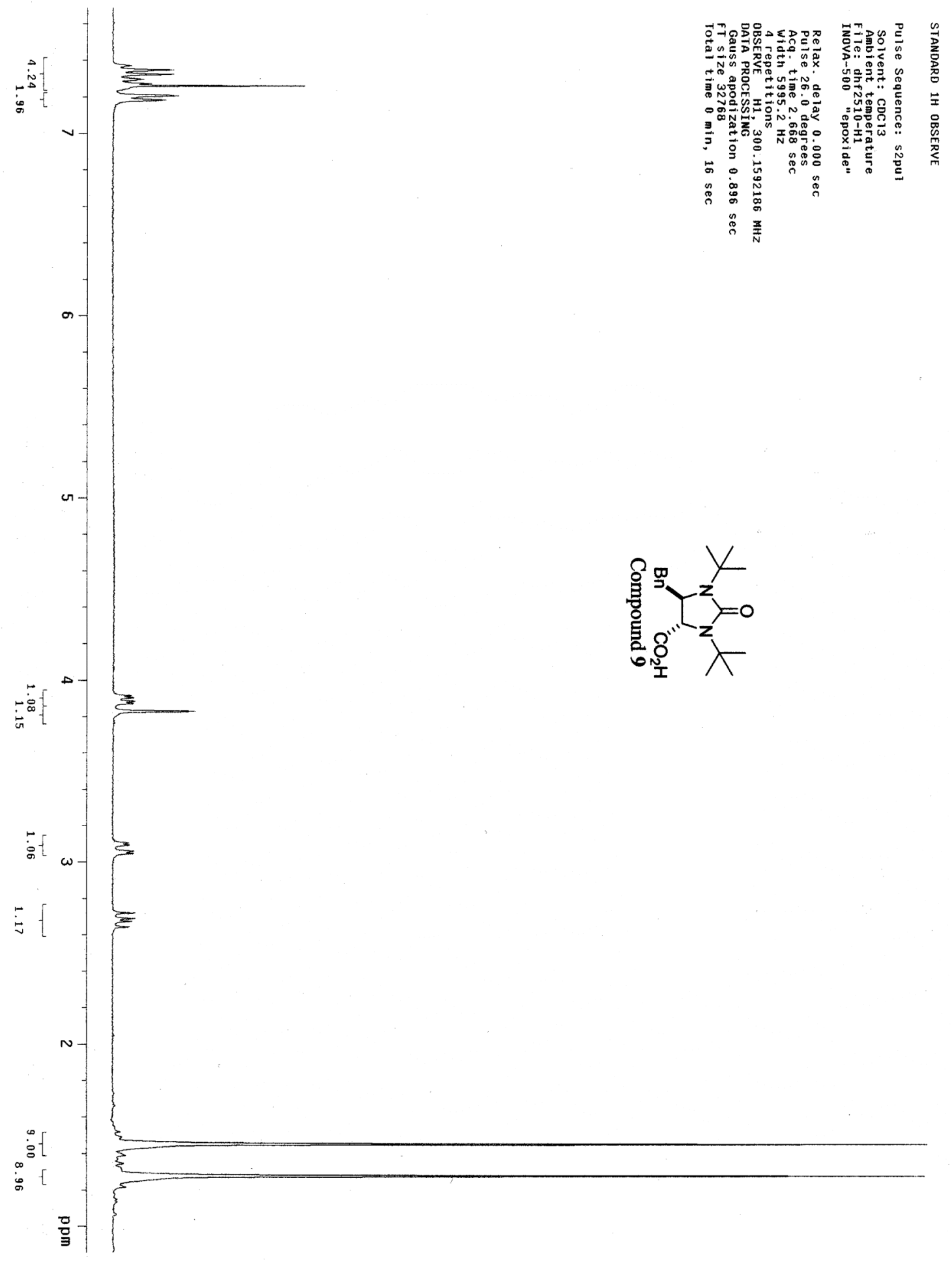




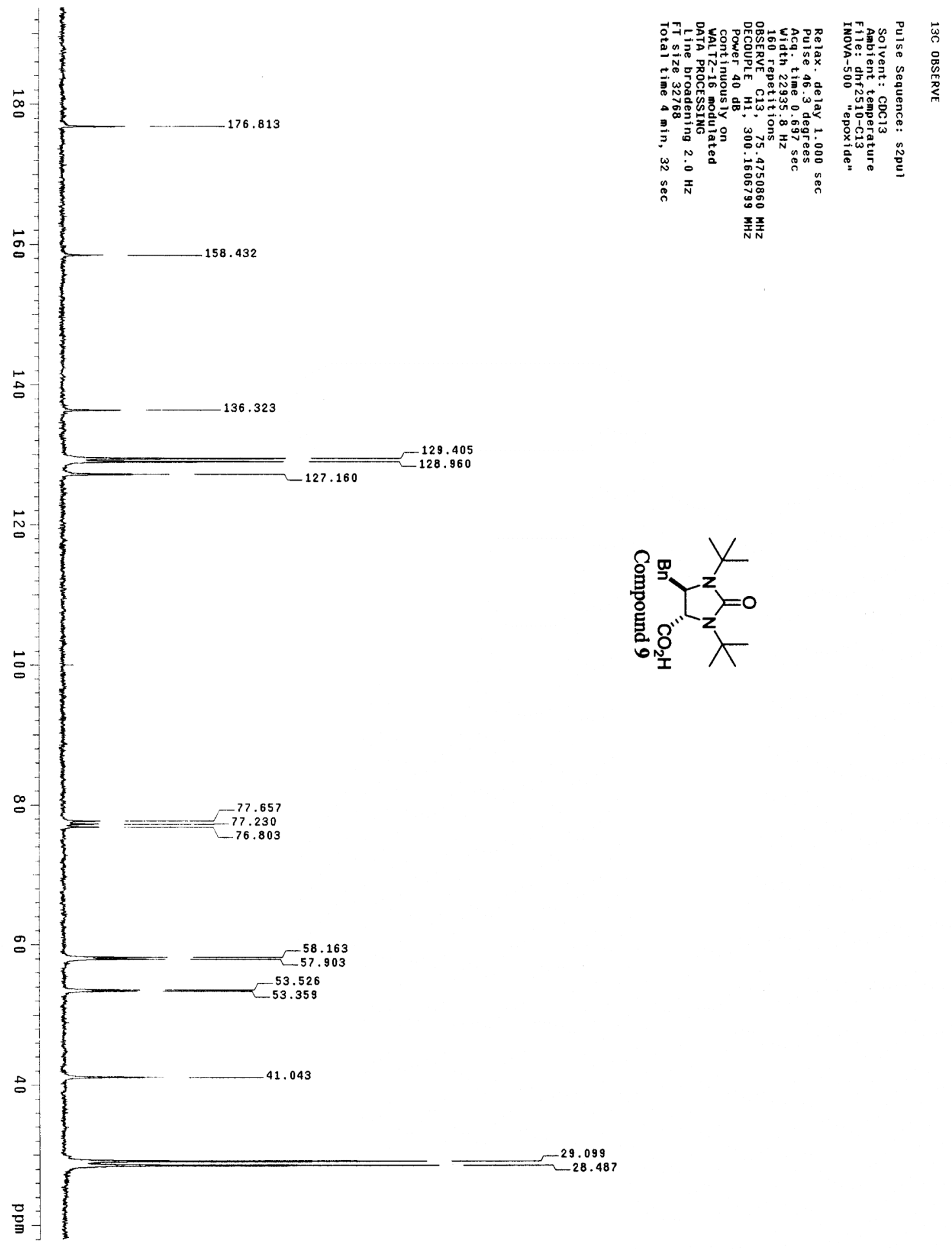




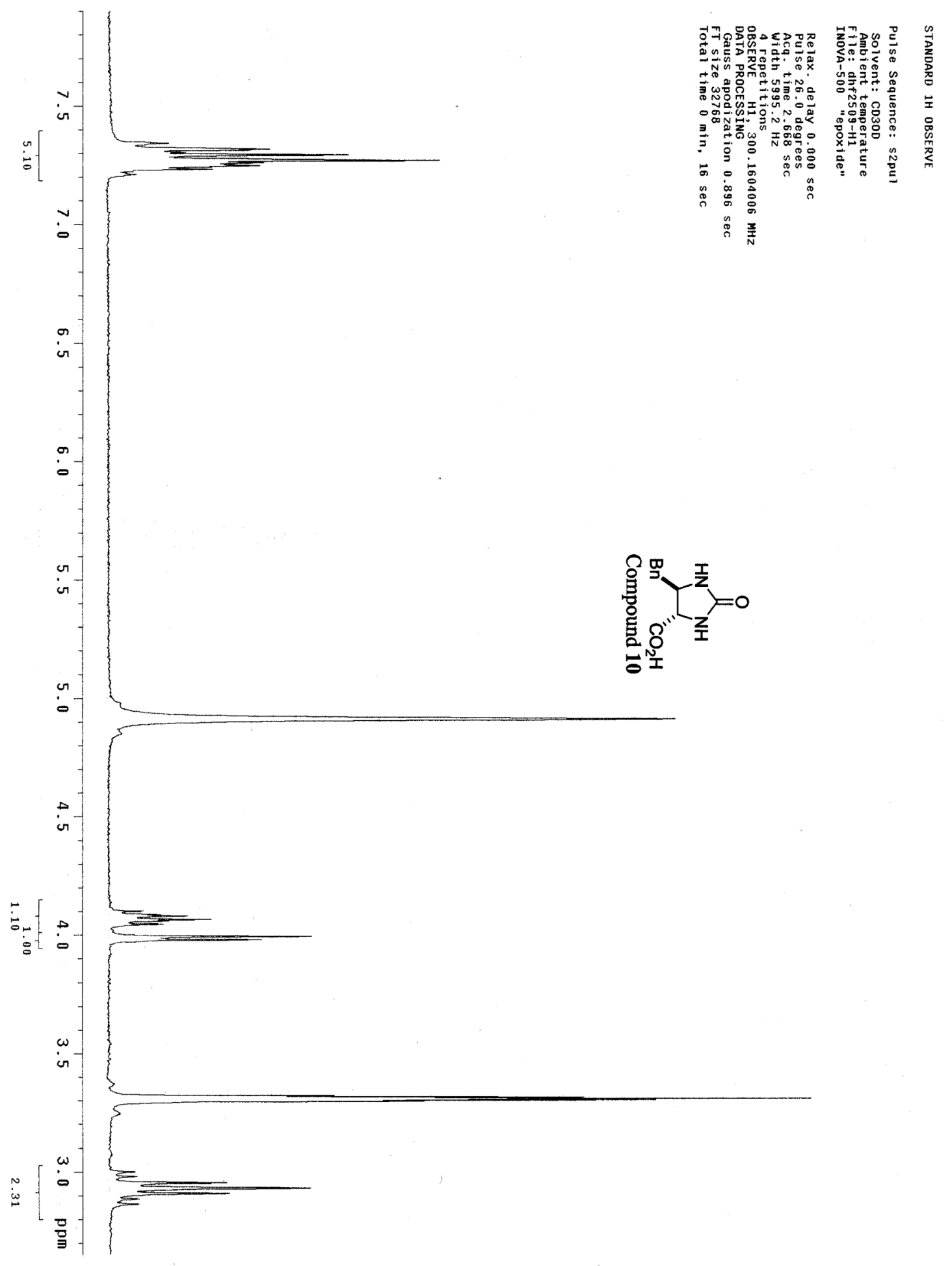




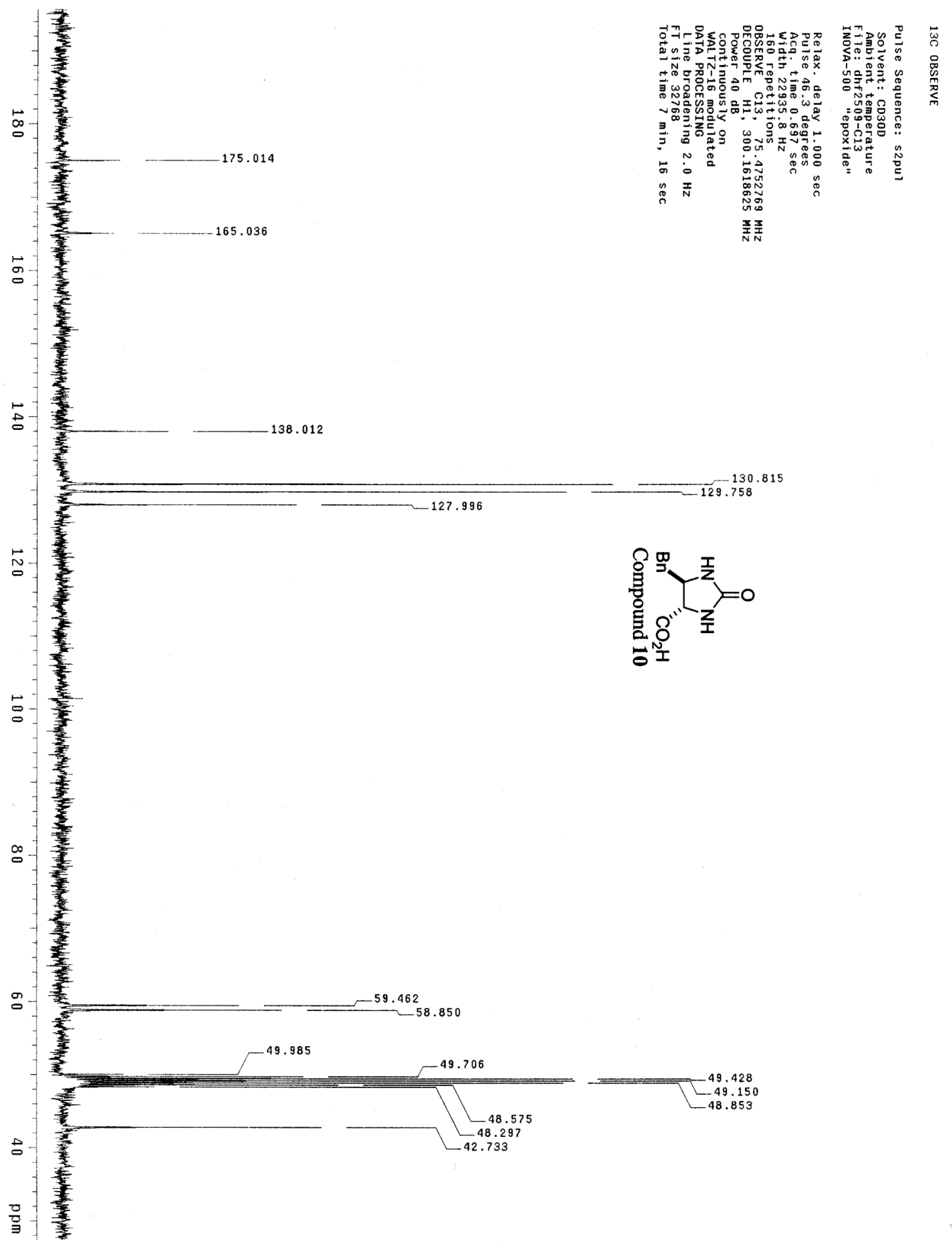



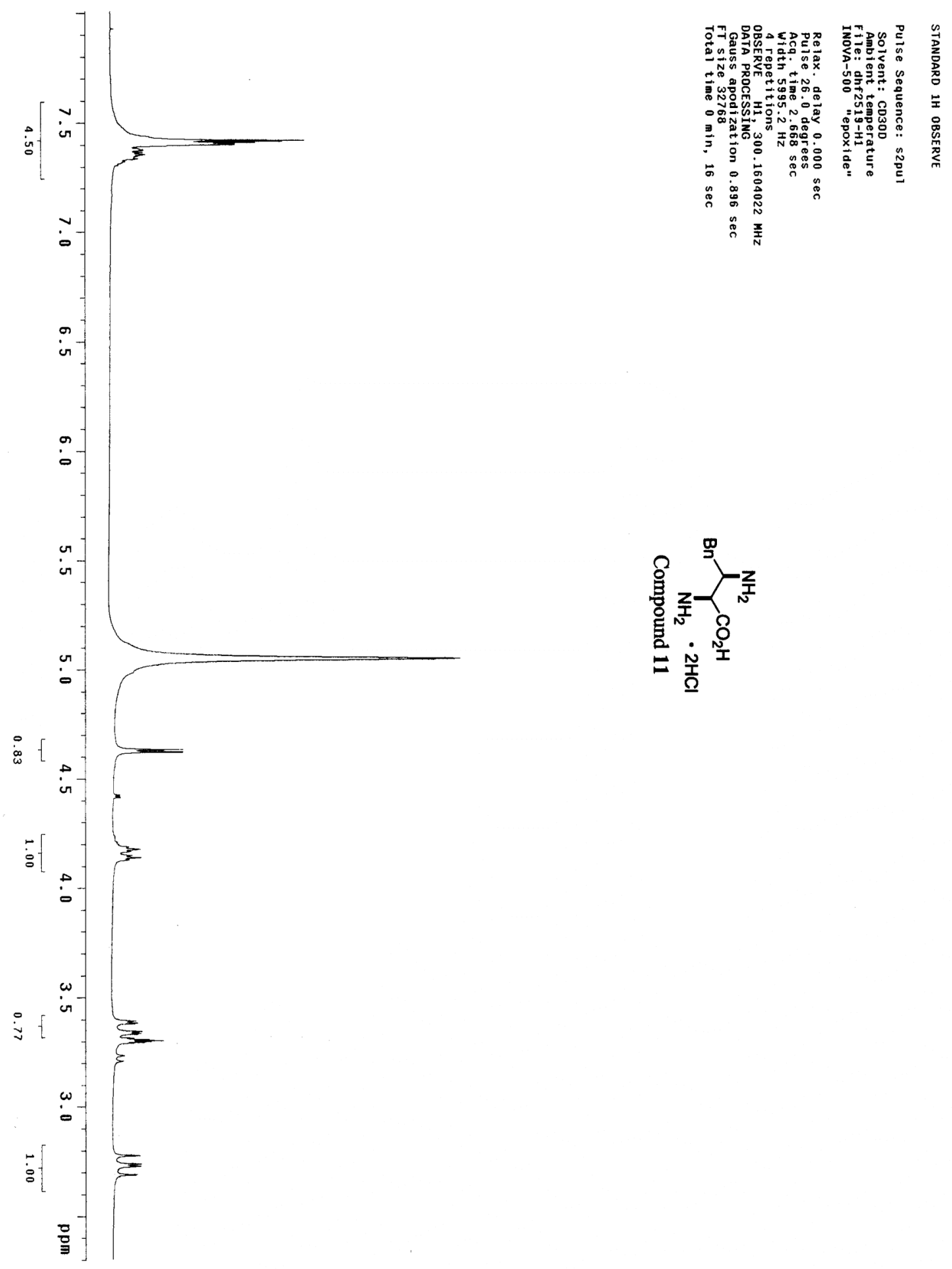


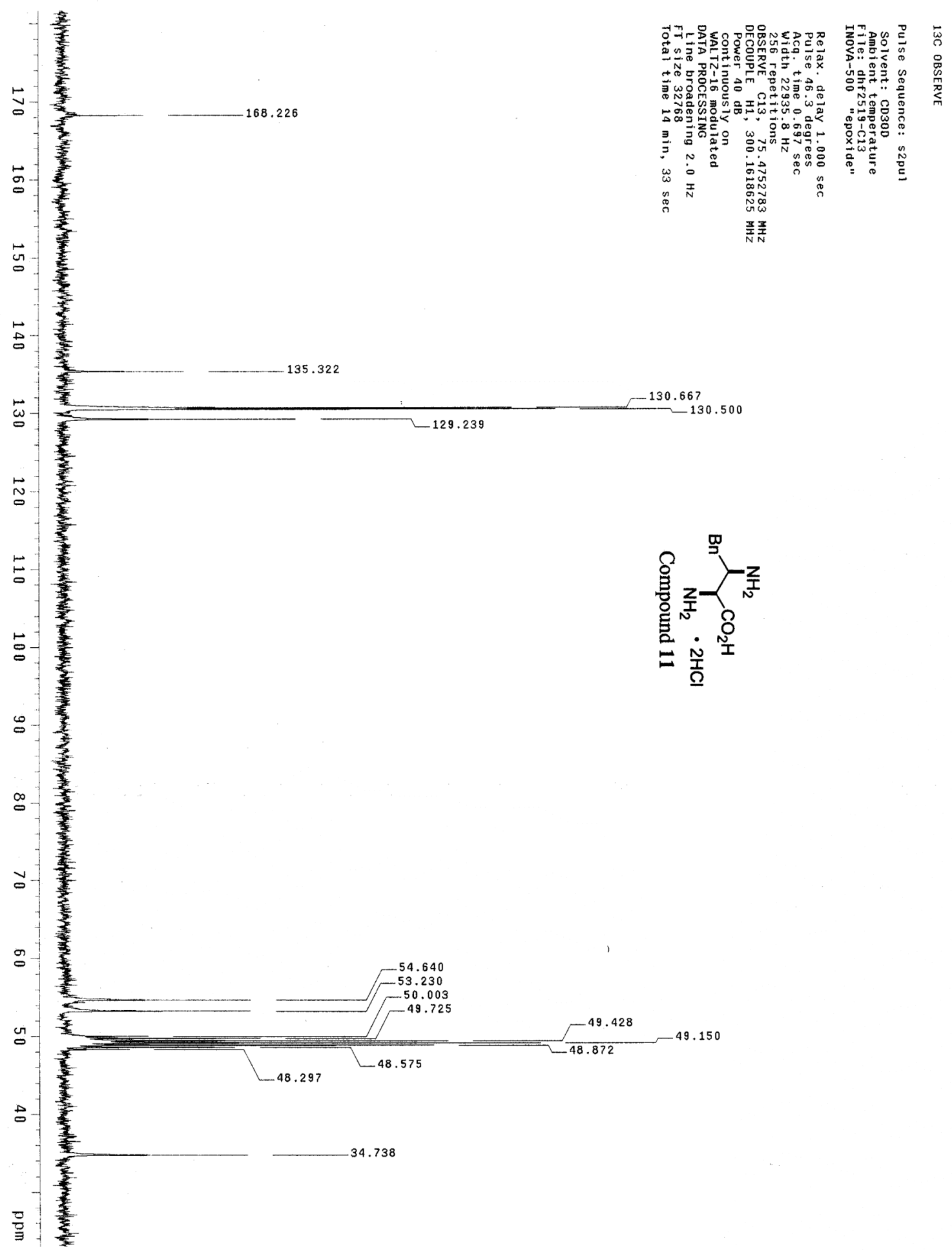




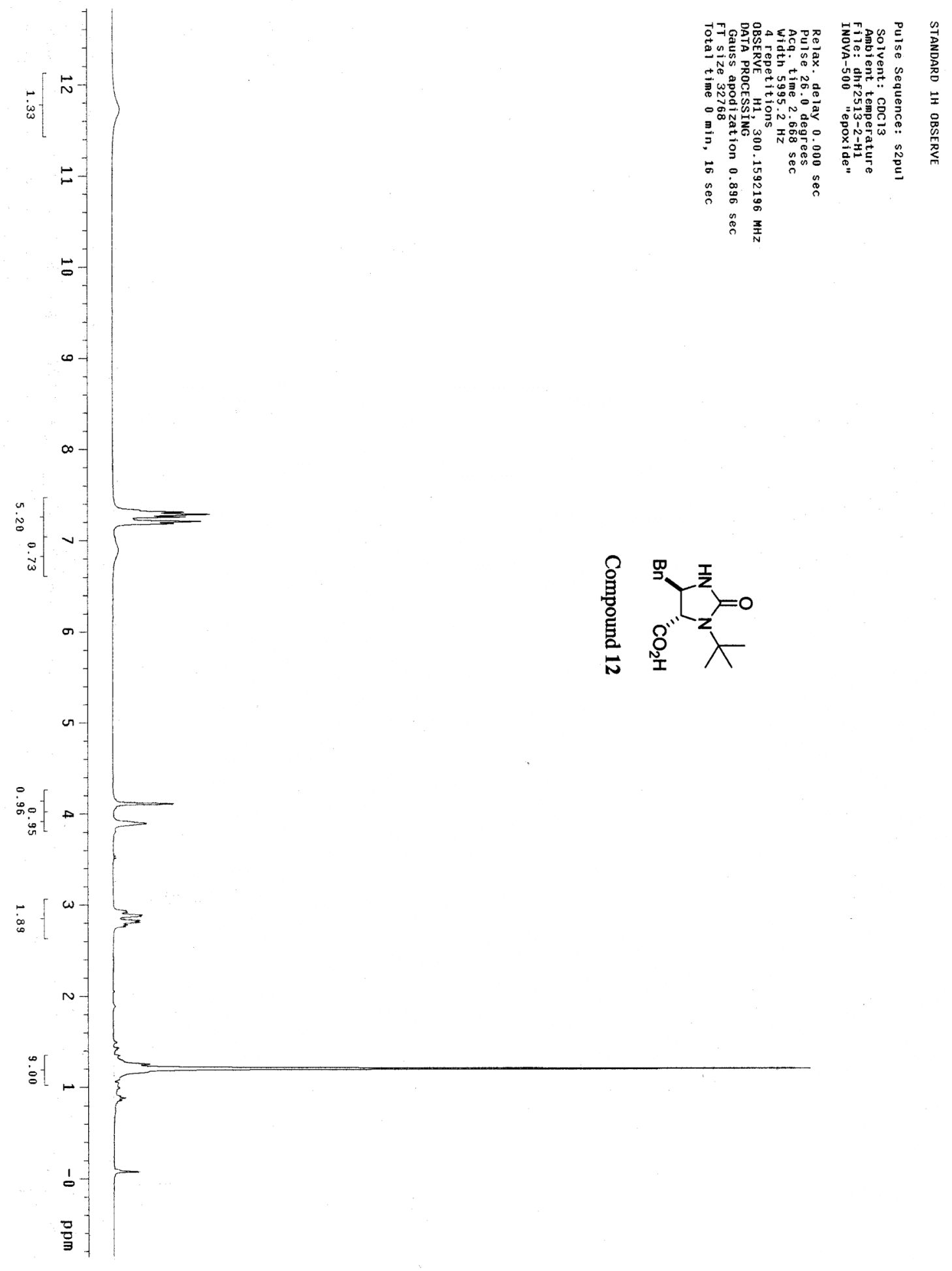




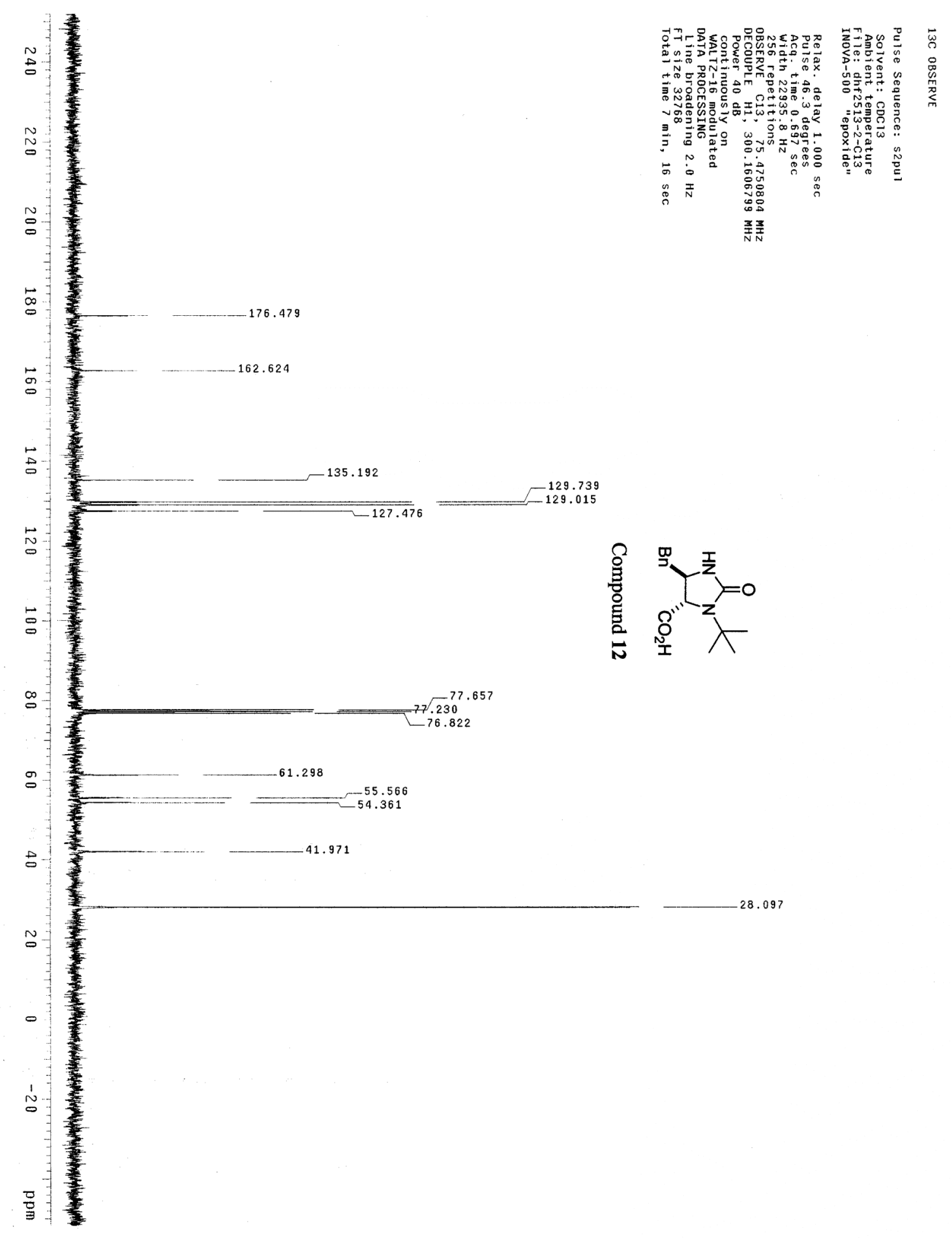

